\title{
Geologic evolution of the Serrinha nucleus granite-greenstone terrane (NE Bahia, Brazil) constrained by U-Pb single zircon geochronology
}

\author{
D.C. Rios ${ }^{\mathrm{a}, *}$, D.W. Davis ${ }^{\mathrm{b}}$, H. Conceição ${ }^{\mathrm{a}}$, W.J. Davis ${ }^{\mathrm{c}}$, M.L.S. Rosa ${ }^{\mathrm{a}}$, A.P. Dickin ${ }^{\mathrm{d}}$ \\ a Laboratory of Applied Petrology (GPA), Instituto de Geociencias, Universidade Federal da Bahia-Rua Caetano Moura 123, 40.210-340, Salvador-BA, Brazil \\ b Jack Satterly Geochronological Laboratory, University of Toronto, Department of Geology, Earth Sciences Centre, 22 Russell Street, Toronto, Ont., Canada M5S 3B1 \\ c Geological Survey of Canada, 601 Booth St., Ottawa, Ont., Canada K1A OE8 \\ d School of Geography and Geology, McMaster University, Hamilton, Ont., Canada L8S 4M1
}

\section{A R T I C L E I N F O}

\section{Article history:}

Received 28 November 2007

Received in revised form

29 September 2008

Accepted 1 October 2008

\section{Keywords:}

Geochronology

$\mathrm{U}-\mathrm{Pb}$

Zircon

Archaean

Palaeoproterozoic

São Francisco Craton

\begin{abstract}
A B S T R A C T
$\mathrm{U}-\mathrm{Pb}$ single zircon crystallization ages were determined using TIMS and sensitive high resolution ion microprobe (SHRIMP) on samples of granitoid rocks exposed in the Serrinha nucleus granite-greenstone terrane, in NE Brazil. Our data show that the granitoid plutons can be divided into three distinct groups. Group 1 consists of Mesoarchaean (3.2-2.9 Ga) gneisses and N-S elongated TTG (Tonalite-TrondhjemiteGranodiorite) plutons with gneissic borders. Group 2 is represented by ca. 2.15 Ga pretectonic calc-alkaline plutons that are less deformed than group 1 . Group 3 is ca. $2.11-2.07 \mathrm{Ga}$, late to post-tectonic plutons (shoshonite, syenite, K-rich granite and lamprophyre). Groups 2 and 3 are associated with the Transamazonian orogeny. Xenocryst ages of $3.6 \mathrm{Ga}$, the oldest zircon yet recorded within the São Francisco craton, are found in the group 3 Euclides shoshonite within the Uauá complex and in the group 2 Quijingue trondhjemite, indicating the presence of Paleoarchaean sialic basement.

Group 1 gneiss-migmatitic rocks (ca. $3200 \mathrm{Ma}$ ) of the Uauá complex constitute the oldest known unit. Shortly afterwards, partial melting of mafic material produced a medium-K calc-alkaline melt, the younger Santa Luz complex (ca. $3100 \mathrm{Ma}$ ) to the south. Subsequent TTG melts intruded in different phases now exposed as N-S elongated plutons such as Ambrósio (3162 $\pm 26 \mathrm{Ma})$, Araci (3072 \pm 2 Ma), Requeijão (2989 $\pm 11 \mathrm{Ma})$ and others, which together form a major part of the Archaean nucleus. Some of these plutons have what appear to be intrusive, but are probably remobilized, contacts with the Transamazonian Itapicuru greenstone belt. The older gneissic rocks occur as enclaves within younger Archaean plutons. Thus, serial additions of juvenile material over a period of several hundred m.y. led to the formation of a stable micro-continent by $2.9 \mathrm{Ga}$. Evidence for Neoarchaean activity is found in the inheritance pattern of only one sample, the group 2 Euclides pluton.

Group 2 granitoid plutons were emplaced at $2.16-2.13 \mathrm{Ga}$ in a continental arc environment floored by Mesoarchaean crust. These plutons were subsequently deformed and intruded by late to post-tectonic group 3 alkaline plutons. This period of Transamazonian orogeny can be explained as a consequence of ocean closure followed by collision and slab break-off. The only subsequent magmatism was kimberlitic, probably emplaced during the Neoproterozoic Braziliano event, which sampled older zircon from the basement.
\end{abstract}

(c) 2008 Elsevier B.V. All rights reserved.

\section{Introduction}

The São Francisco craton, the largest preserved remnant of Archaean and Palaeoproterozoic terranes of the Brazilian shield (Fig. 1A), is well exposed in Bahia State and extends to the southwest into Minas Gerais State (Fig. 1). It has been divided into three crustal blocks: (i) the northern Serrinha nucleus (also called Serrinha block,

\footnotetext{
* Corresponding author. Tel.: +55 712383 8585; fax: +55 7132838591.

E-mail addresses: debora.rios@pq.cnpq.br, debora@cpgg.ufba.br (D.C. Rios).
}

Barbosa and Sabaté, 2004); (ii) the southern Guanambi nucleus; and (iii) the Remanso nucleus, in the central area of the craton (Mascarenhas et al., 1979) (Fig. 1B). These contain a large number of greenstone belts, which have been affected by greenschist to lower amphibolite metamorphism (Cordani et al., 2000). This craton has been regionally stable since the $2.1 \mathrm{Ga}$ Transamazonian orogeny.

The Transamazonian orogeny is characterized by the emplacement of large volumes of granitoid rocks. Lithostratigraphic features, as well as the context of crustal accretion, are still subject to contrasting interpretations. The overall tectonic regime is ascribed 


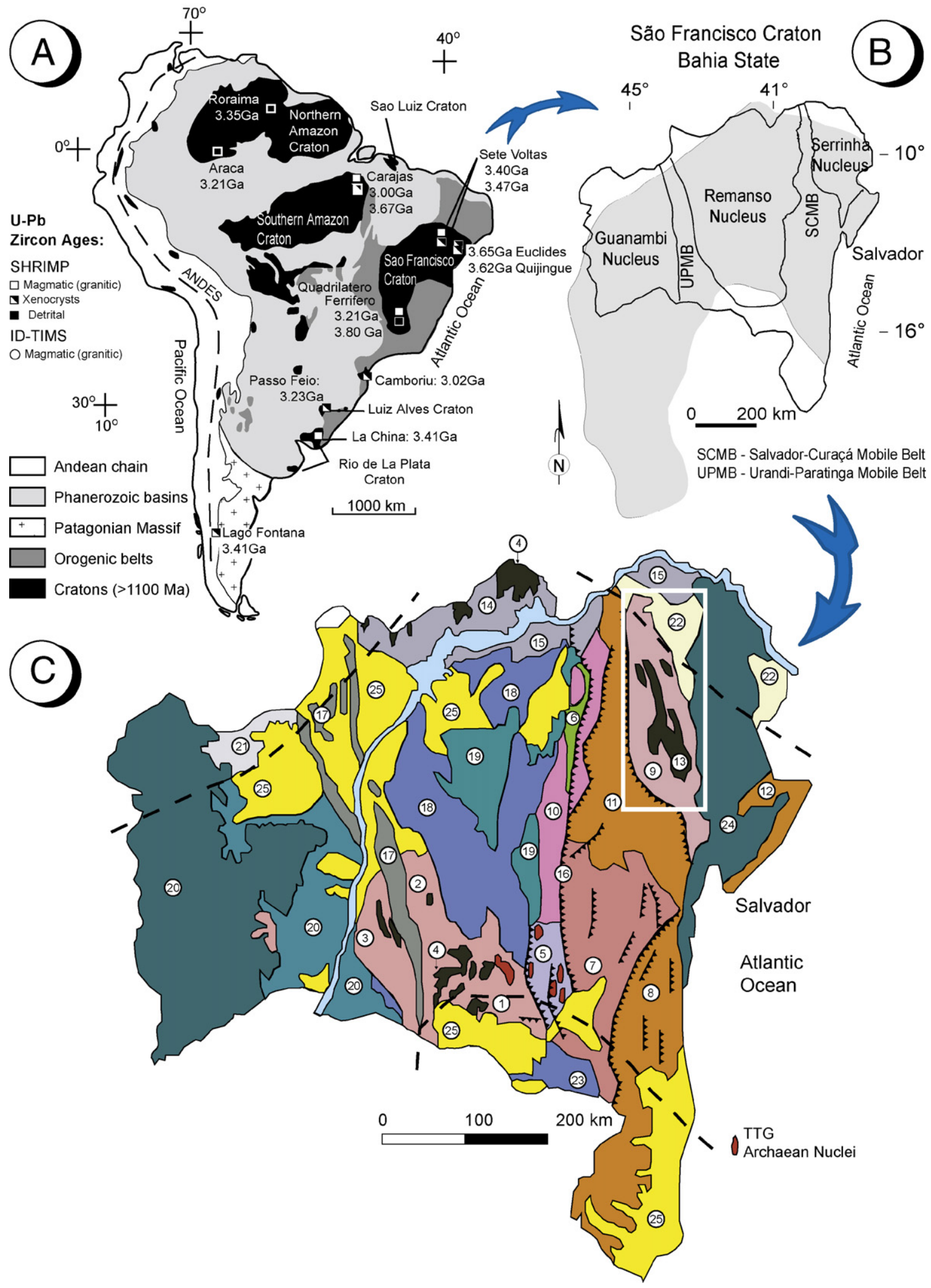

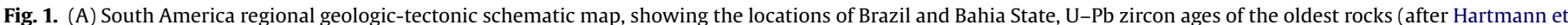

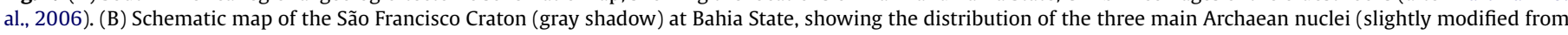

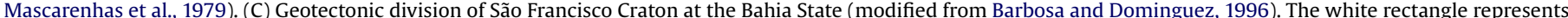

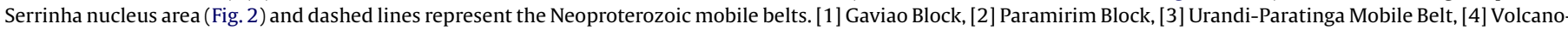

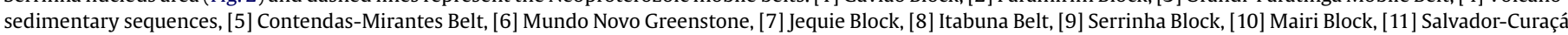

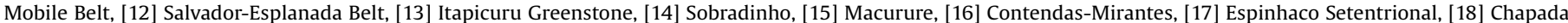

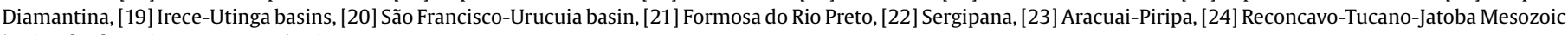
basins, [25] Tercio-Quaternary basins. 


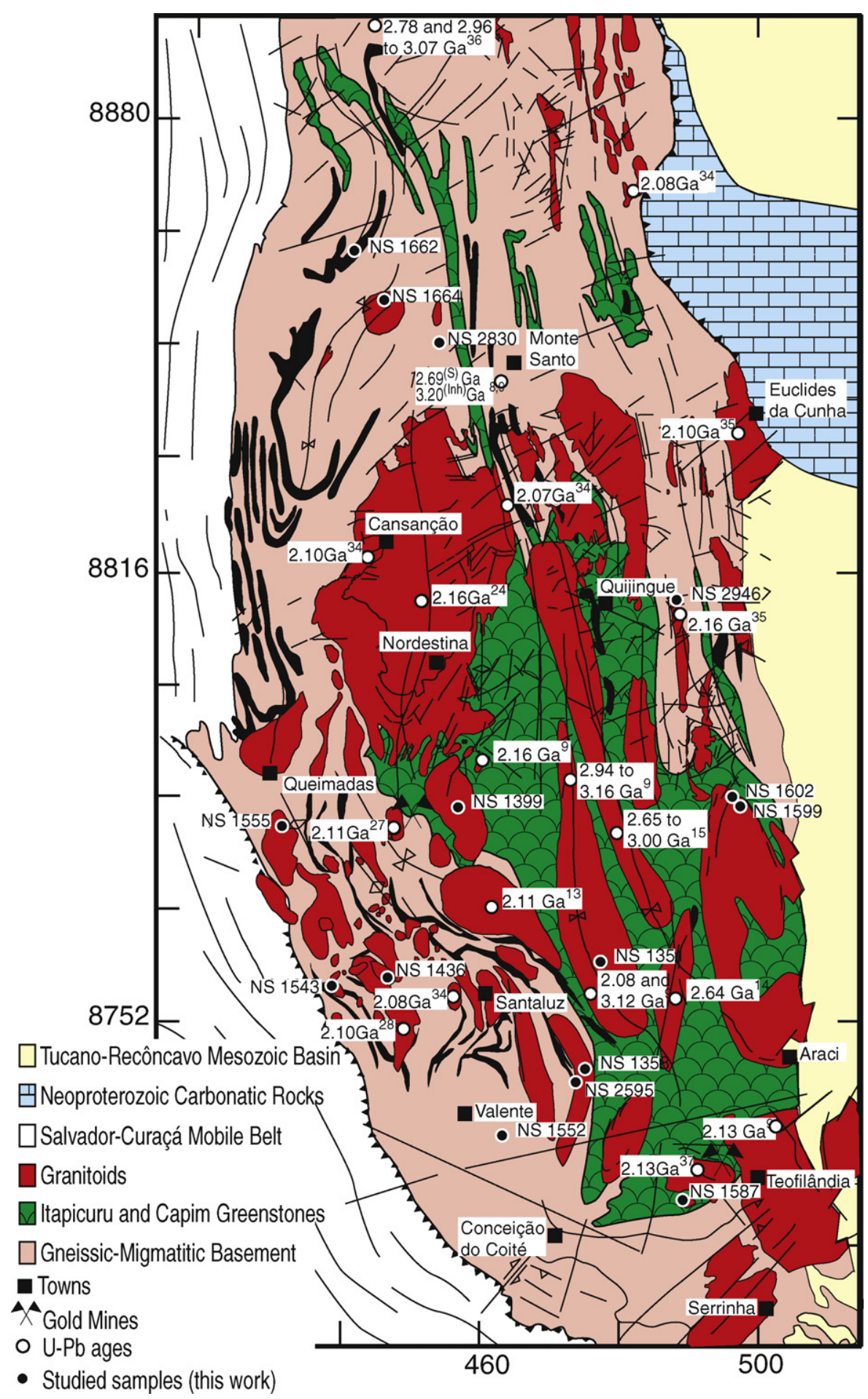

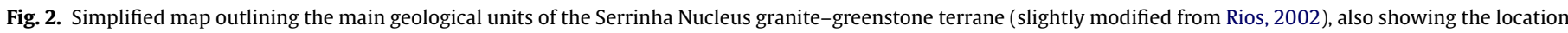

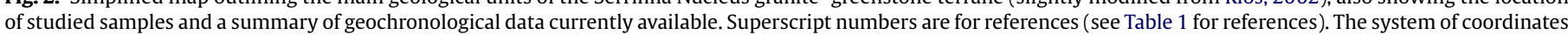
is UTM (SAD 69) and the values are divided by 1000 .

either to actualistic plate tectonic conditions, with dominant collision and thrusting, or to Archaean-type tectonics, with dominant transcurrent shearing and diapirism. One major problem in this debate has been the limited amount of reliable geochronological constraints on these models and, up to now, there were no systematic regional studies of granitoid plutons in the Serrinha nucleus.

This study utilizes sensitive high resolution ion microprobe (SHRIMP) and isotope dilution thermal ionization mass spectrom- etry (ID-TIMS) U-Pb dating methods on granitoid rocks, in order to furnish both high spatial resolution dating of imaged zircon and high age precision where necessary. The aim of this paper is to show the diversity of plutonism in the Serrinha nucleus through the geochronological study of different granitoid groups, to understand the tectonic context in which these rocks formed, and to compare these granitoid suites with similar granite-greenstone terranes worldwide. 


\section{Geological outline}

The Serrinha nucleus represents the northeastern area of the São Francisco craton, Brazil (Mascarenhas et al., 1979). It underlies an area of $>21.000 \mathrm{~km}^{2}$, up to $250 \mathrm{~km}$ long and $70 \mathrm{~km}$ at its widest part, which remained rigid during the Transamazonian orogeny, when it collided with the Salvador-Curaçá mobile belt to the west (Fig. 1C). In the east, it is covered by Neoproterozoic carbonate sedimentary rocks, as well as by the Mesozoic-Cenozoic Tucano-Recôncavo rift basin. In northeast Bahia, the Serrinha nucleus hosts the major concentration of granites of the São Francisco craton and includes the Capim and Itapicuru greenstone belts, as well the Archaean Uauá and Santa Luz granitoid complexes (Fig. 2).

The main lithological units in the Serrinha nucleus granite-greenstone terrane are shown in Fig. 2, on which our dated samples are also located. Table 1 provides a summary of published ages and their interpretation. The terrane consists dominantly of well exposed: (i) Archaean basement, including medium- to high-grade amphibolite facies gneiss-migmatitic rocks and calc-alkaline to Tonalite-Trondhjemite-Granodiorite (TTG) plutons, that are assumed to surround, or enclose, greenstone sequences; (ii) volcano-sedimentary sequences, including the Itapicuru greenstone belt (the most important gold productor of the Bahia State; Silva et al., 2001; Davison et al., 1988), and the Capim greenstone belt (Winge, 1984; Oliveira et al., 1998, 1999); and (iii) Palaeoproterozoic intrusions, which either cut the greenstones or cut both the greenstones and granitoids, and the gneiss-migmatitic basement (Rios et al., 1998, 2000, 2003).

The Itapicuru and Capim greenstone belts (Fig. 2) include from base to top: (i) mafic-ultramafic lava flows, interpreted as E-MORB (enriched mid-ocean ridge basalt) sea floor, and dated at $2.2 \mathrm{Ga}$ (Table 1), consisting of tholeiitic basalt and mafic tuff with associated banded iron formation, chert, and graphitic phyllite; (ii) a bimodal sequence (silica gap between $55 \%$ and $60 \% \mathrm{SiO}_{2}$ ) of andesitic to dacitic flows in the upper units, similar to those of modern active continental margins, and dated at $2080-2178 \mathrm{Ma}$ (Table 1). Both sequences are cut by tonalite-trondhjemite suites (Fig. 2). Intrusive sub-volcanic bodies are represented by rhyodacite, quartz diorite, gabbro and subordinate alkali-feldspar syenite and lamprophyre dykes (formerly mapped as sheared diorite), which occur locally along shear zones with some spatially related to mineralized ore bodies (Xavier, 1991; Barrueto, 1997; Xavier and Foster, 1999). The Itapicuru belt supracrustal pile, which is estimated to be $9.5 \mathrm{~km}$ thick (Davison et al., 1988), shows a number of distinctive features from the Capim belt, including the occurrence of an upper, thick meta-sedimentary unit (conglomerate, sandstone, siltstone and shale, as well as minor carbonate, marble, and banded iron formations).

Most authors recognize three distinct metamorphic events within the Itapicuru greenstone belt (Silva, 1987; Davison et al., 1988; Jardim de Sá, 1982; Chauvet et al., 1997a). An M1 event is related to ocean sea-floor hydrothermal activity and is responsible for spilitization. $\mathrm{M} 2$ developed at greenschist ( $350^{\circ} \mathrm{C}$ at $2 \mathrm{kbar}$ ) to amphibolite $\left(650^{\circ} \mathrm{C}\right.$ at $\left.4 \mathrm{kbar}\right)$ facies conditions and is considered to have been coeval with the emplacement of the main granitic plutons, as the M2 amphibolite facies is restricted to the margins and thermal aureoles developed around intrusions. M3 is a minor event related to contact metamorphism around late-tectonic bodies. All of these events are thought to be Palaeoproterozoic.

Granitoid plutons constitute ca. 30\% of the exposed area and show a wide range of petrographic and geochemical compositions. Previous workers (Matos and Conceição, 1993; Rios et al., 1998) divided the granitoid plutons into five groups; one pretectonic, two syntectonic and two late-tectonic groups within the Transamazonian orogeny. The petrological characteristics and $\mathrm{U}-\mathrm{Pb}$ ages previously available for these rocks are outlined in Table 1 and their locations are shown in Fig. 2.

\section{Sampling and analytical procedures}

The samples were crushed with a jaw crusher followed by a disc mill or shatter box. Mineral separation followed standard procedures using the Wilfley table, the Frantz isodynamic separator and heavy liquids. Zircon grains were hand picked from the least specific magnetic susceptibility fraction available and examined under a binocular microscope. Single zircon grains were selected for analysis based on grouping into populations according to color transparency, magmatic habit, and absence of cracks and inclusions.

\subsection{U-Pb single zircon geochronology by ID-TIMS}

Natural grain surfaces were removed by air abrasion (Krogh, 1982 ) in order to improve concordance. Analyses were carried out at the Jack Satterly Geochronological Laboratory, in Toronto, Canada. Dissolution was performed in small Teflon bombs with HF mixed with a ${ }^{205} \mathrm{~Pb}-{ }^{235} \mathrm{U}$ spike (Krogh, 1973). Dissolved samples were dried, dissolved in $3 \mathrm{~N} \mathrm{HCl}$, and either passed through anion exchange columns with $50 \mu$ l of resin, or loaded directly onto Re filaments with Si-gel and $\mathrm{H}_{3} \mathrm{PO}_{4}$. Samples weighing $<5 \mu \mathrm{g}$ were generally not passed through columns to remove $\mathrm{Zr}$. Isotopic measurements were carried out using a VG354 mass spectrometer equipped with a Daly collector. All common $\mathrm{Pb}$ is assumed to have the isotopic composition of the laboratory blank $\left({ }^{206} \mathrm{~Pb} /{ }^{204} \mathrm{~Pb}-18.221 ;{ }^{207} \mathrm{~Pb} /{ }^{204} \mathrm{~Pb}-15.612\right.$; ${ }^{208} \mathrm{~Pb} /{ }^{204} \mathrm{~Pb}-39.36$; errors of $2 \%$ ). All age calculations and statistical assessments of the data have been done utilizing the geochronological software package ISOPLOT/EX (version 2.00) of Ludwig (2001) and ROMAGE (Davis, 1982).

\subsection{U-Pb geochronology by ion microprobe}

U-Pb SHRIMP analysis were performed at the J.C. Roddick Ion Microprobe Laboratory, of the Geological Survey of Canada (GSC), Ottawa, Canada, using a Sensitive Resolution Ion Microprobe (SHRIMP II). This technique was used to date zircons from rocks that demonstrated complicated histories, in order to distinguish the different periods of zircon growth. The analytical methods of zircon $\mathrm{U}-\mathrm{Pb}-\mathrm{Th}$ age determinations using the SHRIMP of GSC are reported in Stern (1997). The zircon grains were mounted in epoxy together with the zircon standard and grains were then sectioned, polished and photographed. Scanning electron microscope cathodoluminescence imaging was carried out to detect cores, rims and other complexities, which might be present, and to ensure that no areas of mixed age were analyzed. Analyses were performed using a 3$\mathrm{nA}$ primary ion beam and a $30 \mu \mathrm{m}$ spot ( $70 \mu \mathrm{m}$ Kohler aperture), except for a few cases of thin overgrowths where the beam size was reduced to $10 \times 13 \mu \mathrm{m}$. $\mathrm{Pb} / \mathrm{U}$ ratios were determined relative to standard BR266 (559 Ma and 910 ppm U). Standards were analyzed after every five or six unknowns to monitor the stability of calibration. All age calculations and statistical assessments of the data have been done utilizing the geochronological software package ISOPLOT/EX (version 2.00) of Ludwig (1999). Data reduction used the SQUID software (Ludwig, 2001).

\subsection{Nd and Sr isotope analysis}

In selected samples, neodymium and samarium were separated for isotopic analysis by standard cation exchange procedures following $\mathrm{HF}-\mathrm{HNO}_{3}-\mathrm{HCl}$ dissolution of whole-rock powders and 
Table 1

Geochronological overview of the available data for Serrinha nucleus rocks. Anf = amphibole, $\mathrm{Bt}=$ biotite, $\mathrm{Sph}=\mathrm{sphene}, \mathrm{Zr}=\mathrm{zircon}, \mathrm{Mc}=\mathrm{muscovite}, \mathrm{Hb}=\mathrm{hornblende}, \mathrm{Ep}=\mathrm{epidote}, \mathrm{Ap}=\mathrm{apatite}, \mathrm{X}=\mathrm{xenotime}, \mathrm{Fc}=$ fuchsite , $\mathrm{Cl}=$ chlorite, $\mathrm{WR}=$ whole rock, $\mathrm{Inh}=$ inheritance, $\mathrm{M}=$ metamorphism, $\mathrm{D}=$ deformation, $\mathrm{S}=$ sedimentation, $\mathrm{H}=$ hydrothermal zone, $\mathrm{O}=$ overgrowth. When nothing else is specified, $\mathrm{U}-\mathrm{Pb}$ and $\mathrm{Pb}-\mathrm{Pb}$ analysis are for single zircon crystals.

\begin{tabular}{|c|c|c|c|c|c|c|c|c|c|}
\hline \multirow[t]{2}{*}{ Unit } & \multicolumn{9}{|l|}{ Pluton } \\
\hline & $\mathrm{U}-\mathrm{Pb}(\mathrm{Ma})$ & $\mathrm{Pb}-\mathrm{Pb}(\mathrm{Ma})$ & $\mathrm{Rb}-\mathrm{Sr}(\mathrm{Ma})$ & Sri & $\mathrm{K}-\mathrm{Ar}(\mathrm{Ma})$ & $\operatorname{Ar}-\operatorname{Ar}(\mathrm{Ma})$ & $\mathrm{Sm}-\mathrm{Nd}_{\mathrm{WR}}(\mathrm{Ma})$ & $T_{\mathrm{DM}}(\mathrm{Ga})$ & $\varepsilon \mathrm{Nd}$ \\
\hline \multicolumn{10}{|c|}{ Gneiss-migmatitic basement: UAUA (UC) and Santa Luz (SLC) complex } \\
\hline $\begin{array}{l}\text { Caldeirao Quartzite } \\
\text { (UC) }\end{array}$ & $\begin{array}{l}2076 \pm 10^{(\mathrm{M})}, 2687 \pm 16 \\
(\mathrm{~S}), 3051 \pm 13 \text { to } \\
3)^{(\operatorname{lnh})(\mathrm{a}, \mathrm{i})}\end{array}$ & & & & & & & $3.18^{(a)}$ & \\
\hline $\begin{array}{l}\text { Uaua Granodioritic } \\
\text { ortho-gneiss (UC) }\end{array}$ & $\underset{(\mathrm{a}, \mathrm{i})}{2991 \pm 22}$ to $3152 \pm 5$ & & & & $1800-2200^{\mathrm{Anf}, \mathrm{Bt}(\mathrm{b})}$ & & & $3.05^{(e)}$ & $1.61^{(e)}$ \\
\hline $\begin{array}{l}\text { Enderbite Granulite } \\
\text { (UC) }\end{array}$ & $2933 \pm 3^{(a)}$ & & & & & & & $3.13^{(e)}$ & $-0.69^{(\mathrm{e})}$ \\
\hline TTG Retirolandia & $3085^{(\mathrm{g})}$ & & & & & & & $3.12^{(g)}$ & $1.16^{(\mathrm{g})}$ \\
\hline $\begin{array}{l}\text { Enderbite } \\
\text { Orthogranulite Rio das }\end{array}$ & & $3072^{(\mathrm{M})(\mathrm{f}, \mathrm{h})}$ & & & & & & $3.28-3.37^{(\mathrm{e})}$ & -2.22 to $-0.57^{(\mathrm{e})}$ \\
\hline \multicolumn{10}{|l|}{$\begin{array}{l}\text { Orthogranulite Rio das } \\
\text { Vargens/Lagoa da Vaca } \\
\text { (UC) }\end{array}$} \\
\hline Gneiss TTG UC & $\begin{array}{l}2077 \pm 11^{(0)}, 2956 \pm 39 \\
\text { to } 3070 \pm 3^{(\mathrm{ah})}\end{array}$ & & $2700-3100^{(c)}$ & $0.7010-0.7040^{(\mathrm{c})}$ & & & & $2.96-3.26^{(\mathrm{e}, \mathrm{ah})}$ & -1.81 to +1.69 (e,ah) \\
\hline Gneiss SLC & & & $2500-3000^{(d)}$ & & & & & & \\
\hline Amphibolite Dykes & & $2049 \pm 2^{\mathrm{Sph}}$ & $2033 \pm 64$ (d) & $0.7020^{(d)}$ & $2144-1933^{\operatorname{Anf}(c)}$ & & $2895 \pm 170^{(\mathrm{e})}$ & & \\
\hline UC & & $\begin{array}{l}2600-2780 \\
\operatorname{Zr}(\mathrm{i})\end{array}$ & & & & & & & \\
\hline Noritic Dykes UC & & & & & & & $2744 \pm 65$ (e) & & \\
\hline Toleitic Dykes UC & 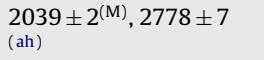 & & & & & & $2586 \pm 66^{(e)}$ & $2.79^{(\mathrm{ah})}$ & \\
\hline \multicolumn{10}{|l|}{ Group 1 granitoids } \\
\hline Ambrosio Gneiss & $\begin{array}{l}2930 \pm 32^{(\mathrm{m})} ; \\
3094 \pm 21,3159 \pm 18^{(\mathrm{i})}\end{array}$ & $1948 \pm 28$ (ab) & $2597 \pm 272^{(1)}$ & $0.7084^{(1)}$ & & & & & \\
\hline Ambrosio Massive & $2077 \pm 2^{(\mathrm{O}, \mathrm{M})}$ & & & & & & & & \\
\hline Granodiorite & $\begin{array}{l}2937 \pm 16,3111 \pm 13 \\
3162 \pm 13^{(i)}\end{array}$ & & & & & & & & \\
\hline Ambrosio Porphyritic & $2063 \pm 55^{(0, M)}$ & & & & & & & & \\
\hline Granodiorite & $2975 \pm 7,3126 \pm 5^{(\mathrm{i})}$ & & & & & & & & \\
\hline Lagoa das Vacas & & $3161 \pm 65^{(h)}$ & & & & & & & \\
\hline \multicolumn{10}{|l|}{ Anorthosite Complex } \\
\hline Rio Capim Tonalite & $2050-3130 \mathrm{Ma}^{(\mathrm{g})}$ & $\begin{array}{l}2650,2900 \\
3000^{(\mathrm{e})}\end{array}$ & $3120^{(e)}$ & & & & & & \\
\hline Pedra Alta & $2650-3000^{(0)}$ & & $1961 \pm 32^{\text {(d) }}$ & $0.7110^{(\mathrm{d})}$ & & & & & \\
\hline Curral & & $\begin{array}{l}2076 \pm 19^{(\mathrm{M})} \\
2468 \pm 3^{(\mathrm{Inh})} \\
2862 \pm 5^{(\mathrm{ab})}\end{array}$ & & & & & & & \\
\hline Poco Grande & $\begin{array}{l}2079 \pm 47^{\mathrm{Mz}(13)} \\
2645 \pm 70^{(\mathrm{n})}\end{array}$ & & & & & $2023 \pm 13^{\mathrm{Mc}(\mathrm{k})}$ & & & \\
\hline Araci & & & $\begin{array}{l}2002 \pm 55 \\
2234 \pm 84^{(d)}\end{array}$ & $0.7049,0.7025^{(4)}$ & & & & & \\
\hline \multicolumn{10}{|c|}{ Greenstone belts Itapicuru and CAPIM } \\
\hline Felsic Dyke Rio & & $2293 \pm 114$ (u) & & & & & & & \\
\hline \multicolumn{10}{|l|}{ Capim } \\
\hline Volcanic Felsic Unit & & $2153 \pm 79^{(u)}$ & & & & & & & \\
\hline \multicolumn{10}{|l|}{ Rio Capim } \\
\hline $\begin{array}{l}\text { Rio Capim Metapelite } \\
\text { Leucogabbre Rio } \\
\text { Capim }\end{array}$ & $2143 \pm 21^{(u)}$ & & & & & & & $2.52^{\text {(a) }}$ & \\
\hline
\end{tabular}


Table 1 (Continued)

\begin{tabular}{|c|c|c|c|c|c|c|c|c|c|}
\hline \multirow[t]{2}{*}{ Unit } & \multicolumn{9}{|l|}{ Pluton } \\
\hline & $\mathrm{U}-\mathrm{Pb}(\mathrm{Ma})$ & $\mathrm{Pb}-\mathrm{Pb}(\mathrm{Ma})$ & $\mathrm{Rb}-\mathrm{Sr}$ (Ma) & Sri & $\mathrm{K}-\mathrm{Ar}(\mathrm{Ma})$ & $\operatorname{Ar}-\operatorname{Ar}(\mathrm{Ma})$ & $\mathrm{Sm}-\mathrm{Nd}_{\mathrm{WR}}(\mathrm{Ma})$ & $T_{\mathrm{DM}}(\mathrm{Ga})$ & $\varepsilon \mathrm{Nd}$ \\
\hline Diorite Rio Capim & $2148 \pm 23$ (u) & \multirow{2}{*}{\multicolumn{2}{|c|}{$\begin{array}{l}2178 \pm 12^{\mathrm{Ep}, \mathrm{Ap}, \mathrm{Zr}(\mathrm{m})} \\
2209 \pm 60^{\mathrm{WR}} \\
(\mathrm{r}, \mathrm{s})\end{array}$}} & & & & & & \\
\hline $\begin{array}{l}\text { Mafic-Basal Unit } \\
\text { Itapicuru }\end{array}$ & $2107 \pm 23^{W R}(\mathrm{n})$ & & & $0.7027^{(\mathrm{a}, \mathrm{s})}$ & & $\begin{array}{l}2080 \pm 5^{\mathrm{Hb}} \\
2108 \pm 5^{\mathrm{Hb}}(\mathrm{r}, \mathrm{s})\end{array}$ & & $2.20^{(s)}$ & $-1.12^{(\mathrm{h})},+4.0^{(\mathrm{s})}$ \\
\hline $\begin{array}{l}\text { Felsic-Intermediate } \\
\text { Unit Itapicuru }\end{array}$ & & $2109 \pm 80^{\mathrm{WR}(\mathrm{r})}$ & $\begin{array}{l}2080 \pm 90 \\
2000 \pm 200 \text { (q) }\end{array}$ & $0.7017^{(q)}$ & $2110 \pm 30^{\mathrm{Mc}}, 2124 \pm 37^{\mathrm{Bt}(\mathrm{s})}$ & & & $2.12^{(s)}$ & $2.0^{(\mathrm{s})}$ \\
\hline Sedimentary Unit & & & $2089 \pm 85^{(n)}$ & $0.7016^{(\mathrm{n})}$ & & & & $2.44-2.49^{\text {(a) }}$ & \\
\hline Itapicuru & & & & & & & & & \\
\hline $\begin{array}{l}\text { Metagabbro sill and } \\
\text { associated } \\
\text { hydrothermal } \\
\text { rocks/Itapicuru } \\
\text { Group } 2 \text { granitoids }\end{array}$ & & & & & & & $2142 \pm 47$ (ae) & & $1.2^{\text {(ae) }}$ \\
\hline $\begin{array}{l}\text { Group } 2 \text { granitolds } \\
\text { Trilhado Undeformed }\end{array}$ & $2152 \pm 6^{\mathrm{Mz}}, 2155 \pm 9^{(\mathrm{i})}$ & & & $0.7017^{(\mathrm{i})}$ & & & & $2.20,2.22^{(i)}$ & $2.41,2.65^{(\mathrm{i})}$ \\
\hline Granodiorite & & & & & & & & & \\
\hline $\begin{array}{l}\text { Nordestina } \\
\text { Trondhjemite }\end{array}$ & & $\begin{array}{l}2100 \pm 10^{\operatorname{Zr}(\mathrm{k})} \\
2155 \pm 9^{(\mathrm{w})}\end{array}$ & $\begin{array}{l}2000^{(\mathrm{m})} ; \\
2114 \pm 103^{(\mathrm{q})}\end{array}$ & $0.7030^{(\mathrm{m})} ; 0.7050^{(\mathrm{q})}$ & & & & $2.24^{(w)}$ & $1.12^{(w)}$ \\
\hline $\begin{array}{l}\text { Teofilandia Tonalite } \\
\text { to Granodiorite }\end{array}$ & $\begin{array}{l}2071 \pm 10^{\circ} \text { and } \\
2130 \pm 7^{(i)}\end{array}$ & & & $0.7025^{(\mathrm{i})}$ & & & & $2.21^{(h)}$ & $2.14^{(i)}$ \\
\hline Barrocas Tonalite & & $2127 \pm 5^{\operatorname{Zr}(\mathrm{k})}$ & & & $2029 \pm 13^{\mathrm{Bt}(\mathrm{k})}$ & & & & \\
\hline Lagoa dos Bois & $2107 \pm 23^{(\mathrm{m})}$ & & & & $2030 \pm 100^{\mathrm{Bt}(\mathrm{v})}$ & & & & \\
\hline Granodiorite & & & & & & & & & \\
\hline Group 3 granitoids & & & & & & & & & \\
\hline Morro do Afonso & $2111 \pm 10^{(z)}$ & $\begin{array}{l}2098 \pm 9 \mathrm{Zr} \text { and } \\
2641 \pm 4^{\text {(Inh }} \\
\mathrm{Zr})(\mathrm{z})\end{array}$ & & $0.7033-0.7042^{(z)}$ & & & & $2.56-2.58^{(z)}$ & -2.36 to $-2.80^{(z)}$ \\
\hline Cansancao & & $2105 \pm 3^{(a b)}$ & $2025 \pm 47$ WR (y) & $0.7033^{(y)}$ & & & & $2.40^{(y)}$ & $-3.00^{(y)}$ \\
\hline Serra do Pintado & $2098 \pm 2^{\text {(aа) }}$ & & & $0.7022^{(a a)}$ & & & & 2.69 (аа) & -4.17 (aа) \\
\hline Morro das & & $2086 \pm 17 \mathrm{Zr}$ (aa) & & & & & & & \\
\hline Agulhas-Bananas & & & & & & & & & \\
\hline Morro do Lopes Type & & & & & $1791 \pm 53^{\mathrm{Bt}(\mathrm{q})}$ & & & & \\
\hline (MLG) Granodiorite & & & & & & & & & \\
\hline Barroquinhas (MLT) & & & $1962^{\mathrm{WR}(\mathrm{x})}$ & $0.7466^{(x)}$ & & & & & \\
\hline Maravilhas (MLT) & $2071 \pm 1^{(\mathrm{af})}$ & & & $0.8081_{\text {(af) }}$ & & & & 3.13 (af) & \\
\hline Santa Luz (MLT) & & & $2064 \pm 25^{\mathrm{WR}(\mathrm{q})}$ & $0.7031^{(q)}$ & & & & & \\
\hline
\end{tabular}


Late intrusions and mineralization

Gold: Scheelita

Fazenda Brasileiro

Mine

Amphibolites,
pegmatites and

pegmatites and

granodioritic dykes

cutting Ambrosio

Quartz-Feldspar

Porphyry Dyke cutting

Teofilandia

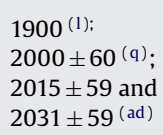

$1900^{(1) ;}$

$2000 \pm 60^{(q)}$
$2015 \pm 59$ and

$2031 \pm 59^{\text {(ad) }}$

0.7024 to $0.7060^{(\mathrm{i})}$

\section{$2050 \pm 4^{\mathrm{Fc}}$}

$2054 \pm 2^{\mathrm{Cl},} 2040$ to

$2065^{\mathrm{Bt}(\mathrm{i})} ; 2031 \pm 4$

to $2083 \pm 4^{\mathrm{BC}}$

$2071 \pm 9^{\circ}, 2128 \pm 8^{(\mathrm{i})}$
1.97 to 2.14 (i)

1.68 to 6.61

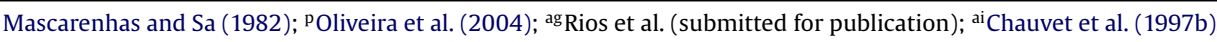

a Oliveira et al. (2002).

b Bastos Leal (1992).

c Bastos Leal et al. (1994).

d Mascarenhas and Garcia (1987).

e Oliveira et al. (1999).

f Paixao et al. (1995)

g Cordani et al. (1999).

h Paixão and Oliveira (1998).

i Mello et al. (2006).

k Alves da Silva et al. (1993).

1 Mascarenhas et al. (1984).

m Gaal et al. (1987).

n Melo et al. (1995).

- Batista et al. (1998).

${ }^{a}$ Brito Neves et al. (1980).

${ }^{\mathrm{r}}$ Silva (1992).

Sliva (1996). (1998).

Oliveira et al. (1998).

Cordani et al. (1969).

w Cruz Filho et al. (2005)

$x$ Pereira (1992).

y Sabate et al. (1990).

${ }^{z}$ Rios et al. (2007).

aa Conceição et al. (2002).

ab Rios et al. (2000).

ac Vasconcelos and Becker (1992).

ad Teixeira (1993).

ae Pimentel and Silva (2003).

af Rios (2002).

ah Oliveira et al. (2000). 
Table 2

SHRIMP data for Serrinha nucleus rocks. Analyzed zircon crystals are colorless, euhedral and inclusion free, except as described above. Pb $\mathrm{b}_{\mathrm{com}}=$ common $\mathrm{Pb}$, considering that they all have the isotopic composition of the lab blank. $\mathrm{Ab}=$ abraded, $\mathrm{zr}=\mathrm{zircon}$ crystal, clr $=$ clear, brk = broked, elong = elongated, transp = transparent, $\mathrm{Hf}=\mathrm{Hf}$ collected, $\mathrm{spr}=$ short prism, lpr =long prism, incl = inclusions, no chem = no chemistry $/$ collumns, irr = irregular shape, round = rounded shape. . The system of coordinates is UTM (SAD 69).

\begin{tabular}{|c|c|c|c|c|c|c|c|c|c|c|c|c|c|c|}
\hline Analysis no. & $\mathrm{U}(\mathrm{ppm})$ & Th (ppm) & ${ }^{232} \mathrm{Th} /{ }^{238} \mathrm{U}^{\mathrm{a}}$ & ${ }^{204} \mathrm{~Pb} /{ }^{206} \mathrm{~Pb}$ & ${ }^{206} \mathrm{~Pb} /{ }^{238} \mathrm{U}$ & $2 \sigma$ & ${ }^{207} \mathrm{~Pb} /{ }^{235} \mathrm{U}$ & $2 \sigma$ & ${ }^{206} \mathrm{~Pb} /{ }^{238} \mathrm{U}(\mathrm{Ma})$ & $1 \sigma$ & ${ }^{207} \mathrm{~Pb} /{ }^{206} \mathrm{~Pb}(\mathrm{Ma})$ & $1 \sigma$ & Disc. $(\%)^{b}$ & Corr. coeff \\
\hline \multicolumn{15}{|c|}{ NS 2830: Maracana basement gneiss-migmatite (UTM 447628 and 8892989) (ca. 3166 e $2079 \mathrm{Ma})^{c}$} \\
\hline 9053-8.1 & 72 & 48 & 0.69 & 0.000184 & .5758 & .0119 & 18.13 & 0.41 & 2931 & 49 & 3041 & 14 & 4 & .926 \\
\hline 9053-15.1 & 111 & 13 & 0.12 & 0.000034 & .5798 & .0148 & 18.08 & 0.48 & 2948 & 60 & 3025 & 10 & 3 & .970 \\
\hline $9053-35.1$ & 117 & 58 & 0.51 & 0.000045 & .5779 & .0108 & 17.86 & 0.36 & 2940 & 44 & 3011 & 12 & 2 & .925 \\
\hline $9053-17.1$ & 161 & 89 & 0.57 & 0.000012 & .5516 & .0114 & 16.41 & 0.35 & 2832 & 47 & 2949 & 9 & 4 & .964 \\
\hline $9053-10.1$ & 579 & 26 & 0.05 & 0.000016 & .3938 & .0070 & 7.01 & 0.13 & 2140 & 33 & 2087 & 7 & -3 & .977 \\
\hline $9053-29.1$ & 427 & 55 & 0.13 & 0.000059 & .3656 & .0060 & 6.50 & 0.11 & 2009 & 28 & 2084 & 10 & 4 & .948 \\
\hline $9053-37.1$ & 340 & 32 & 0.10 & 0.000053 & .3717 & .0063 & 6.52 & 0.12 & 2037 & 30 & 2059 & 10 & 1 & .947 \\
\hline \multicolumn{15}{|c|}{ NS 1351: Ambrosio pluton (UTM 476160 and 8759943$)(3162 \pm 26 \mathrm{Ma})^{d}$} \\
\hline $9060-25.1$ & 61 & 26 & 0.45 & 0.000225 & .6054 & .0143 & 20.87 & 0.53 & 3051 & 57 & 3185 & 15 & 4 & .927 \\
\hline $9060-29.1$ & 306 & 203 & 0.69 & 0.000028 & .6338 & .0107 & 21.63 & 0.38 & 3164 & 42 & 3169 & 8 & 0 & .957 \\
\hline $9060-1.1$ & 51 & 20 & 0.41 & 0.000010 & .6235 & .0141 & 21.18 & 0.52 & 3124 & 56 & 3161 & 15 & 1 & .923 \\
\hline $9060-19.1$ & 64 & 32 & 0.52 & 0.000085 & .6082 & .0132 & 20.61 & 0.48 & 3063 & 53 & 3157 & 14 & 3 & .930 \\
\hline $9060-18.1$ & 88 & 33 & 0.39 & 0.000058 & .6452 & .0173 & 21.72 & 0.60 & 3210 & 68 & 3147 & 12 & -2 & .963 \\
\hline $9060-30.1$ & 53 & 24 & 0.47 & 0.000176 & .6479 & .0147 & 21.77 & 0.57 & 3220 & 58 & 3144 & 20 & -2 & .875 \\
\hline $9060-1.2$ & 1435 & 313 & 0.23 & 0.001076 & .2930 & .0028 & 7.87 & 0.13 & 1657 & 14 & 2783 & 22 & 68 & .593 \\
\hline $9060-9.1$ & 1301 & 14 & 0.01 & 0.006270 & .1377 & .0019 & 2.16 & 0.18 & 832 & 11 & 1861 & 150 & 124 & .167 \\
\hline \multicolumn{15}{|c|}{ NS 1599: Araci pluton (UTM 499063 and 8782049)d } \\
\hline $9061-4.1$ & 55 & 70 & 1.32 & 0.000375 & .3759 & .0095 & 6.49 & 0.22 & 2057 & 45 & 2032 & 39 & -1 & .757 \\
\hline \multicolumn{15}{|c|}{ NS 1602: Araci pluton (UTM 494382 and 8784504)d } \\
\hline 9055-2.1 & 379 & 26 & 0.07 & 0.000034 & .3746 & .0063 & 6.58 & 0.12 & 2051 & 29 & 2063 & 11 & 1 & .941 \\
\hline 9055-3.1 & 220 & 32 & 0.15 & 0.000067 & .3721 & .0066 & 6.53 & 0.13 & 2039 & 31 & 2061 & 13 & 1 & .920 \\
\hline \multicolumn{15}{|c|}{ NS 1555: Requeijao (UTM 433371 and 8779131$)(2989 \pm 11 \mathrm{Ma})^{\mathrm{d}}$} \\
\hline $9057-30.1$ & 104 & 59 & 0.59 & 0.000124 & .6051 & .0122 & 18.46 & 0.40 & 3050 & 49 & 2990 & 12 & -2 & .939 \\
\hline $9057-12.1$ & 110 & 69 & 0.64 & 0.000000 & .5701 & .0111 & 17.38 & 0.36 & 2908 & 46 & 2989 & 11 & 3 & .942 \\
\hline $9057-29.1$ & 228 & 55 & 0.25 & 0.000118 & .5495 & .0096 & 16.59 & 0.30 & 2823 & 40 & 2973 & 8 & 5 & .958 \\
\hline $9057-9.1$ & 210 & 18 & 0.09 & 0.000141 & .5526 & .0100 & 16.46 & 0.31 & 2836 & 41 & 2951 & 10 & 4 & .945 \\
\hline $9057-24.1$ & 413 & 86 & 0.22 & 0.000026 & .5370 & .0092 & 15.45 & 0.27 & 2771 & 39 & 2895 & 7 & 4 & .973 \\
\hline $9057-5.1$ & 749 & 170 & 0.23 & 0.000372 & .4067 & .0067 & 10.21 & 0.19 & 2200 & 31 & 2672 & 13 & 21 & .908 \\
\hline $9057-28.1$ & 619 & 114 & 0.19 & 0.000559 & .2708 & .0045 & 6.74 & 0.13 & 1545 & 23 & 2658 & 17 & 72 & .857 \\
\hline $9057-39.1$ & 55 & 70 & 1.32 & 0.000375 & .1899 & .0033 & 3.28 & 0.09 & 1121 & 18 & 2032 & 39 & 81 & .618 \\
\hline \multicolumn{15}{|c|}{ NS 1399: Eficeas pluton (UTM 456913 and 8781230) $(2163 \pm 8 \mathrm{Ma})^{\mathrm{e}}$} \\
\hline $9062-3.1$ & 60 & 15 & 0.25 & 0.000264 & .3606 & .0114 & 6.79 & 0.24 & 1985 & 54 & 2184 & 30 & 10 & .879 \\
\hline 9062-13.1 & 85 & 22 & 0.27 & 0.000327 & .3849 & .0080 & 7.22 & 0.19 & 2099 & 37 & 2178 & 30 & 4 & .774 \\
\hline $9062-1.1$ & 241 & 67 & 0.29 & 0.000098 & .4062 & .0071 & 7.56 & 0.15 & 2198 & 32 & 2165 & 15 & -1 & .891 \\
\hline $9062-9.1$ & 123 & 32 & 0.27 & 0.000183 & .3937 & .0075 & 7.19 & 0.16 & 2140 & 35 & 2132 & 21 & 0 & .844 \\
\hline \multicolumn{15}{|c|}{ NS 1543: Cipo pluton (UTM 434504 and 8758260$)(2160 \text { e } 2080 \mathrm{Ma})^{\mathrm{e}}$} \\
\hline $9056-1.1$ & 139 & 24 & 0.18 & 0.000046 & .3841 & .0073 & 7.19 & 0.15 & 2095 & 34 & 2175 & 17 & 4 & .885 \\
\hline 9056-5.1 & 139 & 24 & 0.18 & 0.000046 & .3705 & .0072 & 6.94 & 0.15 & 2032 & 34 & 2175 & 17 & 7 & .891 \\
\hline 9056-4.1 & 265 & 120 & 0.47 & 0.000035 & .3975 & .0069 & 7.41 & 0.14 & 2158 & 32 & 2166 & 11 & 0 & .941 \\
\hline 9056-7.1 & 486 & 210 & 0.45 & 0.000045 & .3743 & .0063 & 6.75 & 0.12 & 2050 & 29 & 2107 & 8 & 3 & .963 \\
\hline
\end{tabular}




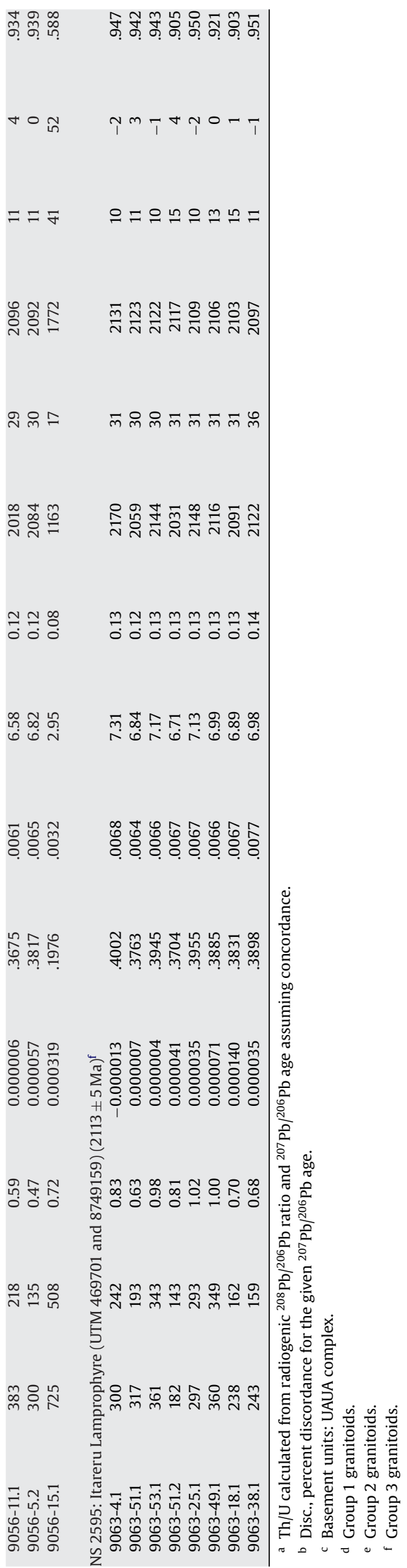

subsequently analyzed on a mass spectrometer at McMaster Isotope Geochronology Laboratory using a VG 354 automated thermal ionization mass spectrometer following established procedures (Holmden and Dickin, 1995). $\mathrm{Rb} / \mathrm{Sr}$ ratios were determined by ICP-MS at Activation Laboratories, Ancaster, Ontario, Canada, using a calibration against international standards. Some data were also checked against "blind" standards previously analyzed by isotope dilution at McMaster. Detection limits are $0.01 \mathrm{ppm}$ for $\mathrm{Sm}$ and $0.05 \mathrm{ppm}$ for $\mathrm{Nd}$. The notations of $\varepsilon \mathrm{Nd}$ and $f_{\mathrm{Sm} / \mathrm{Nd}}$ are defined according to common equations (Faure, 1986). The $\mathrm{Nd}$ and $\mathrm{Sr}$ isotope ratios are normalized to ${ }^{146} \mathrm{Nd} /{ }^{144} \mathrm{Nd}=0.7219$ and ${ }^{86} \mathrm{Sr} /{ }^{88} \mathrm{Sr}=0.1194$, respectively. During the course of this work, the mean value obtained for ${ }^{143} \mathrm{Nd} /{ }^{144} \mathrm{Nd}$ from La Jolla was $0.51185 \pm 0.00002(2 \sigma)$ and for ${ }^{87} \mathrm{Sr} /{ }^{86} \mathrm{Sr}$ from NBS 987 was $0.71030 \pm 0.00004(2 \sigma)$, in agreement with recommended values for these standards. The $\mathrm{Sm} / \mathrm{Nd}$ spike has been calibrated against several rock standards from different isotope labs, and gives a $\mathrm{Sm} / \mathrm{Nd}$ ratio of $0.2280 \pm 0.0002$ for the USGS standard BCR-1. The decay constant for ${ }^{87} \mathrm{Rb}\left(1.42 \times 10^{-11} / \mathrm{yr}\right)$ is taken from Davis et al. (1977) and for ${ }^{147} \mathrm{Sm}\left(6.54 \times 10^{-12} / \mathrm{yr}\right)$, from Lugmair and Marti (1978).

\section{Results}

Isotopic analysis are given in Table 2 for SHRIMP and in Table 3 for ID-TIMS. Rb-Sr and Sm-Nd data are presented in Table 4. Uncertainties in the isotopic ratios and ages in the data tables (and in the error ellipses in the plotted data) are reported at the $2 \sigma$ level. Where ages are calculated from combined data or from regression analysis, errors are quoted at $95 \%$ confidence limits. Results are presented in chronological order from oldest to youngest. Detailed descriptions of the units sampled are given in Rios (2002).

\subsection{Pretectonic Mesoarchaean granitoid rocks}

The lithologies of the Serrinha nucleus basement are represented by the Uauá complex in the north, which has been correlated with the Congo craton (Bellieni et al., 1995), and by the Santa Luz complex in the south. These complexes are composed of gneissic migmatite with amphibolitic layers and banded gneiss and gneiss with garnet and sillimanite, which seems to surround the gneissic migmatite. These rocks were metamorphosed to amphibolite-facies, have tonalitic-granodioritic and quartzfeldspatic compositions, and include subordinate amphibolite and meta-ultramafic rock types (Bellieni et al., 1995). They were partially remobilized during the Transamazonian orogeny (Beurlen, 1970; Winge, 1984; Bastos Leal, 1992). Numerous less eroded granitic plutons cut the gneissic lithologies.

The general orientation of the gneissic fabric is $315^{\circ}-330^{\circ}$ dipping steeply SW. Aerial photography and Landsat images reveal complex structural relationships, suggesting the existence of several deformational events. Amphibolite rocks occur as enclaves, or as concordant and discordant layers. There are many hypotheses to explain the genesis of these mafic rocks, from marginal sediments (Inda et al., 1976) to mafic meta-volcanic rocks from the greenstone belts (Mascarenhas et al., 1979; Melo et al., 1995). The greenschist facies metamorphism affects both the gneiss and amphibolite and is here interpreted as an effect of Palaeoproterozoic regional metamorphism.

Based on whole-rock litho-geochemical studies (see Rios, 2002 for details), there are no significant differences between the gneisses of the Santa Luz and Uauá complexes. All the gneissic rocks show a small range of silica contents $\left(69.9 \%<\mathrm{SiO}_{2}<75.8 \%\right)$, being predominantly potassic $\left(0.3>\mathrm{K}_{2} \mathrm{O} / \mathrm{Na}_{2} \mathrm{O}>3.1\right.$, ratios from 0.9 to 3.8 
Table 3

ID-TIMS data for Serrinha nucleus rocks. Ab = abraded, $\mathrm{zr}=$ zircon, $\mathrm{cl}=$ clear, incl = inclusion, no chem = no chemistry, brk = broked, elong = ellongated, frag = fragment, col = collected, rnd = rounded, lpr = long prism, irreg = irregular

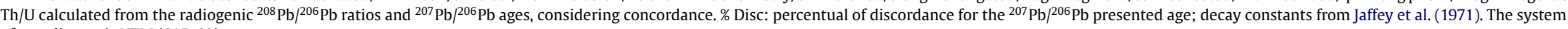
of coordinates is UTM (SAD 69).

\begin{tabular}{|c|c|c|c|c|c|c|c|c|c|c|c|c|c|c|}
\hline Zr. no. & Analyzed fraction description & Weight (mg) & $\mathrm{U}(\mathrm{ppm})$ & $\mathrm{Th} / \mathrm{U}$ & $\mathrm{Pb}_{\mathrm{Com}}(\mathrm{pg})$ & ${ }^{207} \mathrm{~Pb} /{ }^{204} \mathrm{~Pb}$ & ${ }^{206} \mathrm{~Pb} /{ }^{238} \mathrm{U}$ & $2 \sigma$ & ${ }^{207} \mathrm{~Pb} /{ }^{235} \mathrm{U}$ & $2 \sigma$ & ${ }^{207} \mathrm{~Pb} /{ }^{206} \mathrm{~Pb}$ age $(\mathrm{Ma})$ & $2 \sigma$ & \% Disc. & Corr. coeff. \\
\hline \multicolumn{15}{|c|}{ NS 1552: Valente basement gneiss (UTM 457994 and 8739173) $(3102 \pm 5$ Ma) } \\
\hline 3 & $1 \mathrm{ab}$ zr, pink, clr, brk, seems to have core, no chem & 0.0030 & 87.5 & 0.54 & 1.07 & 2289.8 & 0.6136 & 0.0071 & 20.001 & 0.233 & 3096 & 2 & 0.5 & 0.99541 \\
\hline 1 & $1 \mathrm{clr}$, ab zr, black incl, no chem & 0.0030 & 49.3 & 0.39 & 0.67 & 2075.0 & 0.6124 & 0.0023 & 19.931 & 0.080 & 3094 & 2 & 0.6 & 0.96537 \\
\hline 2 & 1 small, clr, ab zr, no chem & 0.0005 & 142.4 & 0.27 & 1.68 & 284.8 & 0.4901 & 0.0018 & 13.667 & 0.061 & 2845 & 3 & 11.6 & 0.89223 \\
\hline 4 & 1 ab brown zr, elong, clr, no chem & 0.0010 & 141.4 & 0.61 & 0.74 & 617.2 & 0.3775 & 0.0018 & 6.748 & 0.032 & 2093 & 4 & 1.6 & 0.90631 \\
\hline \multicolumn{15}{|c|}{ NS 1662: Amphibolite layer (443339 and 8858224) (2078 $\pm 2 \mathrm{Ma})^{\mathrm{a}}$} \\
\hline 2 & 1 ab zr fragment, shiny, pale pink, clr, transp, $\mathrm{Hf}$ & 0.0050 & 54.1 & 0.28 & 1.88 & 466.5 & 0.3788 & 0.0009 & 6.716 & 0.020 & 2079 & 3 & 0.5 & 0.87253 \\
\hline 3 & 1 ab zr, core?, anedric, round, pale pink, clr, $\mathrm{Hf}$ & 0.0150 & 27.8 & 0.28 & 1.37 & 965.6 & 0.3792 & 0.0010 & 6.723 & 0.019 & 2079 & 2 & 0.4 & 0.91452 \\
\hline 1 & 1ab zr, frosted fragment, cloudy & 0.0080 & 25.8 & 0.28 & 3.24 & 214.6 & 0.3797 & 0.0018 & 6.729 & 0.040 & 2078 & 6 & 0.2 & 0.85235 \\
\hline 4 & $1 \mathrm{ab} z \mathrm{zr}$, flat, from core dish, pale pink, clr, no chem & 0.0020 & 43.6 & 0.28 & 1.17 & 250.0 & 0.3813 & 0.0020 & 6.746 & 0.040 & 2075 & 5 & -0.4 & 0.89250 \\
\hline \multicolumn{15}{|c|}{ NS 2830: Maracana basement gneiss-migmatite (overgrowths) (UTM 447628 and 8892989$)(2070 \pm 32 \mathrm{Ma})^{\mathrm{a}}$} \\
\hline 1 & $1 \mathrm{Ab}$ tip, pale brown & 0.0010 & 1074 & 0.10 & 9.33 & 369.5 & 0.3739 & 0.0014 & 6.607 & 0.028 & 2073 & 3 & 1.4 & 0.90002 \\
\hline 2 & 1 Ab tip, pale brown & 0.0010 & 381.1 & 0.09 & 2.88 & 419.1 & 0.3723 & 0.0015 & 6.560 & 0.029 & 2068 & 3 & 1.6 & 0.90898 \\
\hline \multicolumn{15}{|c|}{ NS 1351: Ambrosio pluton (UTM 476160 and 8759943$)(3068 \pm 6 \mathrm{Ma})^{\mathrm{b}}$} \\
\hline 2 & 1 big rounded zr, clr & 0.0020 & 70.3 & 0.68 & 1.18 & 1219.5 & 0.6343 & 0.0027 & 21.642 & 0.095 & 3169 & 2 & 0.1 & 0.96944 \\
\hline 3 & 1 abr zr overgrowth & 0.0015 & 4.2 & 0.25 & 0.49 & 132.4 & 0.6094 & 0.0110 & 19.654 & 0.366 & 3079 & 9 & 0.5 & 0.95011 \\
\hline 1 & 1 ab zr, big crack, clr, not well ab, no chem & 0.0040 & 242.1 & 0.18 & 6.85 & 1054.1 & 0.4902 & 0.0017 & 15.796 & 0.059 & 3078 & 2 & 19.9 & 0.95943 \\
\hline 5 & 1 ab zr, cracks?, cores?, no chem & 0.0010 & 146.6 & 0.17 & 1.65 & 364.2 & 0.3009 & 0.0010 & 8.451 & 0.034 & 2856 & 3 & 46.0 & 0.90154 \\
\hline 6 & $1 \mathrm{zr}$, crack free?, melt incl on middle & 0.0080 & 49.5 & 0.45 & 23.74 & 80.4 & 0.3054 & 0.0010 & 8.565 & 0.084 & 2853 & 16 & 45.1 & 0.23433 \\
\hline 4 & 1 ab zr overgrowht, no chem & 0.0030 & 30.9 & 0.21 & 3.26 & 49.1 & 0.1398 & 0.0006 & 2.534 & 0.049 & 2117 & 28 & 64.0 & 0.81159 \\
\hline \multicolumn{15}{|c|}{ NS 1599: Araci pluton (UTM 499063 aand 8782049) $(\sim 3070 \mathrm{Ma})^{\mathrm{b}}$} \\
\hline 3 & $1 \mathrm{ab}$ zr, angular, not well abraded, clr, no chem & 0.0005 & 88.7 & 0.53 & 0.82 & 438.7 & 0.5307 & 0.0026 & 16.863 & 0.086 & 3055 & 3 & 12.5 & 0.91998 \\
\hline 1 & $1 \mathrm{ab} z \mathrm{zr}$, clr, shine, cloudy spot on surface & 0.0030 & 48.3 & 0.08 & 13.87 & 91.8 & 0.5396 & 0.0376 & 15.672 & 1.442 & 2911 & 103 & 5.4 & 0.71359 \\
\hline 2 & 1 abr zr, clr, with a cloudy crack(?), no chem & 0.0020 & 138.6 & 0.62 & 2.31 & 373.8 & 0.2260 & 0.0013 & 6.409 & 0.035 & 2872 & 5 & 59.7 & 0.84538 \\
\hline \multicolumn{15}{|c|}{ NS 1555: Requeijao pluton (UTM 433371 and 8779131) ( 2870 Ma) ${ }^{\mathrm{b}}$} \\
\hline 2 & 1 abr zr overgrowth, no chem & 0.0005 & 157.1 & 0.79 & 1.59 & 279.7 & 0.4169 & 0.0027 & 11.461 & 0.081 & 2821 & 5 & 24.1 & 0.91541 \\
\hline 4 & 1 abr zr overgrowth, no chem & 0.0005 & 177.2 & 1.44 & 1.46 & 357.0 & 0.4427 & 0.0025 & 12.065 & 0.068 & 2807 & 5 & 18.9 & 0.85536 \\
\hline 1 & 5 small zr grains, pale pink, clr & 0.0040 & 86.5 & 0.54 & 2.83 & 572.4 & 0.3654 & 0.0018 & 9.789 & 0.051 & 2779 & 3 & 32.2 & 0.95128 \\
\hline 3 & 1 ab zr overgrowth, no chem & 0.0005 & 144.5 & 0.39 & 1.75 & 223.8 & 0.4232 & 0.0017 & 10.843 & 0.055 & 2706 & 4 & 18.9 & 0.85485 \\
\hline 5 & 1 no ab zr, flat, spr, cracked, altered, $\mathrm{Hf}$ & 0.0050 & 76.6 & 0.27 & 6.18 & 188.1 & 0.2647 & 0.0032 & 5.981 & 0.073 & 2496 & 9 & 44.0 & 0.89526 \\
\hline \multicolumn{15}{|c|}{ NS 1399: Eficeas pluton (UTM 456913 and 8781230) $(2163 \pm 5 \mathrm{Ma})^{\mathrm{c}}$} \\
\hline 2 & $1 \mathrm{ab} z r$, clr, elong, with small black incl, no chem & 0.0004 & 91.0 & 0.27 & 0.70 & 188.3 & 0.3837 & 0.0022 & 7.097 & 0.050 & 2153 & 7 & 3.2 & 0.82405 \\
\hline 1 & $1 \mathrm{clr}$ ab zr, no chem & 0.0005 & 215.4 & 0.34 & 0.92 & 391.9 & 0.3745 & 0.0011 & 6.910 & 0.025 & 2149 & 4 & 5.3 & 0.78810 \\
\hline 3 & 1 brown ab zr, little cloudy, no chem & 0.0005 & 371.9 & 0.31 & 0.32 & 1546.6 & 0.3165 & 0.0010 & 5.692 & 0.020 & 2104 & 2 & 18.0 & 0.95691 \\
\hline \multicolumn{15}{|c|}{ NS 1543: Cipo pluton (UTM 434504 and 8758260) $(2164 \pm 2 \mathrm{Ma})^{\mathrm{c}}$} \\
\hline 1 & $1 \mathrm{ab} z r$, clr, rounded, no chem & 0.0015 & 127.6 & 0.52 & 1.79 & 384.2 & 0.3983 & 0.0011 & 7.421 & 0.025 & 2166 & 3 & 0.2 & 0.85658 \\
\hline 2 & $1 \mathrm{ab} \mathrm{zr}$, clr, rounded, melt inclusion, $\mathrm{Hf}$ & 0.0040 & 129.2 & 0.52 & 3.58 & 506.4 & 0.3930 & 0.0044 & 7.313 & 0.081 & 2163 & 5 & 1.4 & 0.97071 \\
\hline 3 & $1 \mathrm{ab}$ zr, pink, clr, rounded, no chem & 0.0020 & 68.9 & 0.23 & 0.71 & 651.9 & 0.3823 & 0.0019 & 7.039 & 0.037 & 2145 & 3 & 3.1 & 0.95391 \\
\hline 4 & 1 ab zr, elong, clr, no chem & 0.0020 & 53.5 & 0.57 & 0.69 & 508.2 & 0.3812 & 0.0015 & 6.795 & 0.030 & 2088 & 3 & 0.4 & 0.92134 \\
\hline \multicolumn{15}{|c|}{ NS 1358: Itareru Shoshonitic pluton (UTM 468465 and 8741159) $(2106 \pm 2 \mathrm{Ma})^{\mathrm{d}}$} \\
\hline 3 & $1 \mathrm{clr}$, ab zr, with bubble incl, cloudy, no chem & 0.0010 & 100.7 & 0.32 & 0.97 & 367.1 & 0.3925 & 0.0016 & 7.298 & 0.032 & 2162 & 4 & 1.5 & 0.84965 \\
\hline 2 & 1 ab zr tip, clr, pale brown, no chem & 0.0005 & 288.4 & 0.48 & 2.63 & 184.8 & 0.3680 & 0.0014 & 6.625 & 0.034 & 2106 & 7 & 4.7 & 0.64229 \\
\hline 1 & 1 brown ab zr tip, broked, turbid, no chem & 0.0010 & 350.9 & 0.43 & 1.19 & 947.0 & 0.3758 & 0.0010 & 6.765 & 0.021 & 2106 & 2 & 2.7 & 0.92662 \\
\hline 4 & 1 pale pink, clr, ab zr, rounded, no chem & 0.0030 & 32.0 & 0.42 & 1.21 & 264.6 & 0.3747 & 0.0013 & 6.745 & 0.031 & 2106 & 4 & 3.0 & 0.84950 \\
\hline
\end{tabular}




\begin{tabular}{|c|c|c|c|c|c|c|c|c|c|c|c|c|c|c|}
\hline \multicolumn{15}{|c|}{ NS 1664: Pedra Vermelha Massif (UTM 445681 and 8854669$)(2080 \pm 8 \mathrm{Ma})^{\mathrm{d}}$} \\
\hline 2 & 1 small, clr, ab zr, no chem & 0.0003 & 116.6 & 0.52 & 0.54 & 217.8 & 0.3790 & 0.0020 & 6.713 & 0.045 & 2077 & 8 & 0.3 & 0.75945 \\
\hline 4 & 1 ab zr frag, pink, clr, little cloudy, incl, no chem & 0.0005 & 121.3 & 0.39 & 1.12 & 172.5 & 0.3527 & 0.0030 & 6.226 & 0.058 & 2071 & 12 & 6.9 & 0.70993 \\
\hline 3 & 1 ab zr, pink, shine, clr, no chem & 0.0010 & 161.2 & 0.62 & 1.28 & 317.8 & 0.3013 & 0.0008 & 5.156 & 0.018 & 2016 & 4 & 17.9 & 0.79400 \\
\hline 1 & 1 pale pink, irreg shape, ab zr frag, clr, no chem & 0.0010 & 180.1 & 0.32 & 2.04 & 218.5 & 0.2887 & 0.0076 & 4.938 & 0.135 & 2015 & 15 & 21.4 & 0.95362 \\
\hline \multicolumn{15}{|c|}{ NS 1587: Barroquinhas Massif (UTM 491748 and 8723673$)(2073 \pm 1 \mathrm{Ma})^{\mathrm{d}}$} \\
\hline 2 & 2 small zr, irreg shaped, elong, round, clr, pale pink, no chem & 0.0030 & 186.4 & 0.68 & 0.58 & 3000.8 & 0.3776 & 0.0014 & 6.681 & 0.026 & 2075 & 2 & 0.5 & 0.95286 \\
\hline 3 & $1 \mathrm{ab}$ zr with cracks, clr, pink, $\mathrm{Hf}$ & 0.0200 & 183.7 & 0.67 & 2.49 & 4553.0 & 0.3737 & 0.0009 & 6.607 & 0.018 & 2074 & 1 & 1.5 & 0.95252 \\
\hline 1 & 1 big single zr grain, pink, clr, rounded, $\mathrm{Hf}$ & 0.0100 & 159.4 & 0.64 & 1.28 & 3911.2 & 0.3796 & 0.0009 & 6.708 & 0.018 & 2073 & 2 & -0.1 & 0.93937 \\
\hline 4 & 1 ab zr, cracks, brown, no chem & 0.0040 & 310.6 & 0.54 & 0.99 & 3856.8 & 0.3724 & 0.0027 & 6.578 & 0.049 & 2072 & 2 & 1.8 & 0.98741 \\
\hline 5 & $1 \mathrm{ab}$ zr, clr, pale pink, well rounded, no chem & 0.0010 & 145.1 & 0.72 & 1.77 & 263.9 & 0.3696 & 0.0015 & 6.514 & 0.032 & 2068 & 4 & 2.3 & 0.86016 \\
\hline \multicolumn{15}{|c|}{ NS 1436: Fazenda Bananas Massif (UTM 444530 and 8759162) $(2072 \pm 1 \mathrm{Ma})^{\mathrm{d}}$} \\
\hline 4 & 1 brown zr grain, elong, clr, zoned, no chem & 0.0010 & 409.0 & 0.56 & 0.84 & 1462.8 & 0.3613 & 0.0008 & 6.422 & 0.017 & 2083 & 2 & 5.3 & 0.93636 \\
\hline 1 & 2 geminated brown zr, clr, no chem & 0.0030 & 218.0 & 0.57 & 1.02 & 1981.7 & 0.3746 & 0.0014 & 6.619 & 0.026 & 2073 & 2 & 1.2 & 0.95796 \\
\hline 2 & 1 pale pink, large zr grain, clr, irreg & 0.0070 & 153.8 & 0.99 & 0.96 & 3488.6 & 0.3768 & 0.0010 & 6.654 & 0.019 & 2072 & 2 & 0.6 & 0.93595 \\
\hline 3 & 1 clr zr grain, small, pale pink, rounded & 0.0010 & 53.7 & 2.18 & 1.95 & 83.8 & 0.3095 & 0.0016 & 5.320 & 0.059 & 2024 & 16 & 16.1 & 0.58464 \\
\hline
\end{tabular}

a Basement units: UAUA and Santa Luz complex.

b Group 1 granitoids.

c Group 2 granitoids.

d Group 2 granitoids.

\section{Table 4}

$\mathrm{Rb}-\mathrm{Sr}$ and $\mathrm{Sm}-\mathrm{Nd}$ data for Serrinha nucleus rocks.

\begin{tabular}{|c|c|c|c|c|c|c|c|c|c|c|c|c|c|c|c|c|c|c|}
\hline $\begin{array}{l}\text { Sample } \\
\text { number }\end{array}$ & $\begin{array}{l}\text { Age } \\
\text { (Ga) }\end{array}$ & Rock type & $\begin{array}{l}\text { Sm } \\
(\mathrm{ppm})\end{array}$ & $\begin{array}{l}\mathrm{Nd} \\
(\mathrm{ppm})\end{array}$ & ${ }^{143} \mathrm{Nd} /{ }^{144} \mathrm{Nd}$ & ${ }^{147} \mathrm{Sm} /{ }^{144} \mathrm{Nd}$ & $\begin{array}{l}T_{\text {CHUR }} \\
(\mathrm{Ga})\end{array}$ & $\begin{array}{l}T_{\mathrm{DM}} \\
(\mathrm{Ga})\end{array}$ & $E_{\mathrm{Nd}(0)}$ & $E_{\mathrm{Nd}(\mathrm{T})}$ & $f_{\mathrm{Sm} / \mathrm{Nd}}$ & $\begin{array}{l}{ }^{147} \mathrm{Sm} / \\
{ }^{144} \mathrm{Nd}_{\mathrm{sam}}\end{array}$ & $I_{\mathrm{Nd}}$ & $\begin{array}{l}\mathrm{Rb} \\
(\mathrm{ppm})\end{array}$ & $\begin{array}{l}\text { Sr } \\
(\mathrm{ppm})\end{array}$ & ${ }^{87} \mathrm{Rb} /{ }^{86} \mathrm{Sr}$ & ${ }^{87} \mathrm{Sr} /{ }^{86} \mathrm{Sr}$ & $I_{\mathrm{Sr}}$ \\
\hline 1536 & 2.79 & Capim Diorite & 5.54 & 23.1 & 0.511830 & 0.1449 & 2.37 & 2.75 & -15.76 & 2.89 & -0.21 & 0.16 & 0.509159 & 71 & 509 & 0.4040 & 0.71574 & 0.699395 \\
\hline 1599 & 3.07 & $\begin{array}{l}\text { Araci Monzo- } \\
\text { granite }\end{array}$ & 5.65 & 34.2 & 0.510718 & 0.0997 & 3.00 & 3.12 & -37.45 & 0.93 & -0.41 & 0.12 & 0.508696 & 204 & 129 & 4.6350 & 0.83671 & 0.630183 \\
\hline 1381 & 3.08 & $\begin{array}{l}\text { Pedra Alta } \\
\text { Trondhjemite }\end{array}$ & 2.02 & 14.5 & 0.510362 & 0.0842 & 3.03 & 3.17 & -44.40 & 0.23 & -0.52 & 0.09 & 0.508650 & 62 & 480 & 0.3740 & 0.71672 & 0.700011 \\
\hline 1399 & 2.16 & $\begin{array}{l}\text { Eficeas Biotite- } \\
\text { Trondhjemite }\end{array}$ & 4.98 & 28.1 & 0.511441 & 0.1068 & 2.02 & 2.30 & -23.35 & 1.64 & -0.41 & 0.12 & 0.509919 & 75 & 120 & 1.8130 & & \\
\hline 1630 & 2.16 & $\begin{array}{l}\text { Quijingue } \\
\text { Granodiorite }\end{array}$ & 0.94 & 6.9 & 0.510423 & 0.0822 & 2.93 & 3.27 & -43.21 & -11.57 & -0.53 & 0.09 & 0.509256 & 76 & 476 & 0.4630 & 0.71952 & 0.705133 \\
\hline 1613 & 2.10 & $\begin{array}{l}\text { Euclides } \\
\text { Syeno-Granite }\end{array}$ & 13.81 & 88.9 & 0.510880 & 0.0939 & 2.62 & 2.87 & -34.29 & -6.64 & -0.50 & 0.10 & 0.509583 & 236 & 129 & 5.3710 & 0.85636 & 0.694021 \\
\hline 1650 & 2.08 & $\begin{array}{l}\text { Araras } \\
\text { Monzonite }\end{array}$ & 45.40 & 334.0 & 0.510656 & 0.0820 & 2.42 & 2.96 & -38.66 & -8.12 & -0.57 & 0.09 & 0.509535 & 190 & 1110 & 0.4960 & 0.72001 & 0.705171 \\
\hline 1548 & 2.10 & $\begin{array}{l}\text { Serra do } \\
\text { Pintado Syenite }\end{array}$ & 18.54 & 113.9 & 0.511068 & 0.0984 & 2.37 & 2.69 & -30.63 & -4.07 & -0.47 & 0.11 & 0.509709 & 199 & 1040 & 0.5550 & 0.71896 & 0.702185 \\
\hline 957 & 2.10 & $\begin{array}{l}\text { Morro do } \\
\text { Afonso Syenite }\end{array}$ & 19.89 & 111.7 & 0.511289 & 0.1078 & 2.30 & 2.56 & -26.31 & -2.36 & -0.45 & 0.11 & 0.509800 & 235 & 1764 & 0.3861 & 0.71567 & 0.703994 \\
\hline 1587 & 2.07 & $\begin{array}{l}\text { Barroquinhas } \\
\text { Granite }\end{array}$ & 7.26 & 45.8 & 0.510723 & 0.0958 & 2.59 & 3.13 & -37.33 & -10.54 & -0.46 & 0.11 & 0.509415 & 259 & 93 & 8.2380 & 0.93432 & 0.688217 \\
\hline 1642 & 2.07 & $\begin{array}{l}\text { Maravilhas } \\
\text { Granodiorite }\end{array}$ & 5.53 & 38.1 & 0.510944 & 0.0877 & 3.06 & 2.67 & -33.04 & -4.07 & -0.52 & 0.09 & 0.509748 & 187 & 155 & 3.5260 & 0.80812 & 0.702887 \\
\hline
\end{tabular}



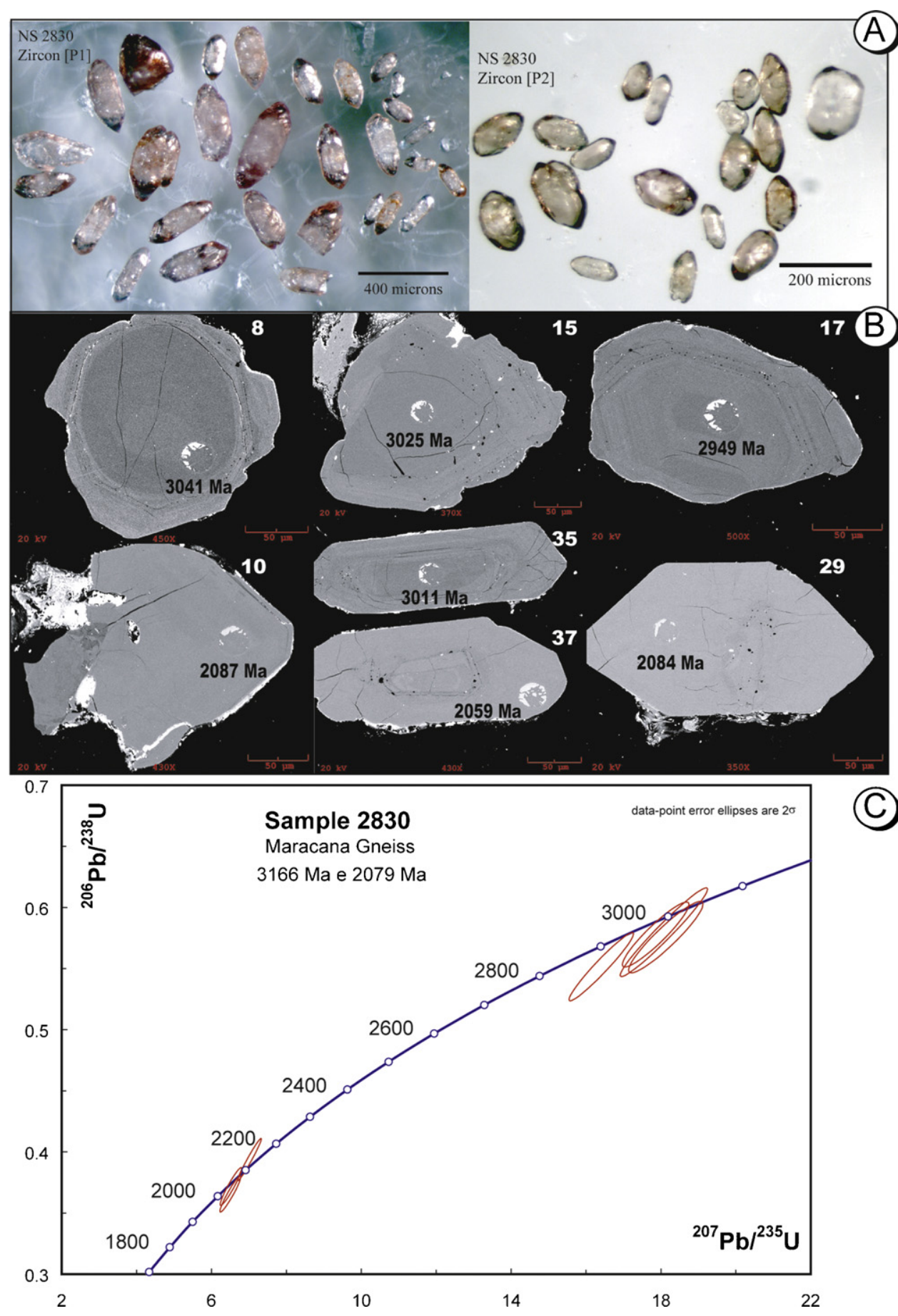

(C)

Fig. 3. Sample 2830-Migmatite-gneiss Maracana, Uauá complex. (A) Zircon population. (B) BSE images of analyzed crystals. (C) SHRIMP Concordia diagram.

predominate) and peraluminous. They correspond to granodiorites and sub-alkaline granites, mostly of TTG tendency. The REE patterns are LREE enriched, showing small anomalies of Eu (positive and negative) and similarity with Archaean TTG suites.

Rios et al. (2008) presented SHRIMP ages of ca. 3300 Ma for an enclave of the gneissic-migmatitic basement related to the Uauá complex, collected near a fault contact with the Quijingue pluton (Fig. 2). These authors identified rounded and transparent zircons with cores and overgrowths of Mesoarchaean age (data scatter between about $3072 \mathrm{Ma}$ and $3314 \mathrm{Ma}$ ). The youngest data are associated with low $\mathrm{Th} / \mathrm{U}$ ratios, which they interpreted as an
Archaean high-grade metamorphic event. This gneiss is interpreted as a crustal remnant that underwent a complex history during the Mesoarchaean and whose zircon was relatively unaffected by the Transamazonian event.

The amphibolites have chemical similarities with tholeiites, although some of them show a calc-alkaline tendency. These are sodic $\left(0.1>\mathrm{K}_{2} \mathrm{O} / \mathrm{Na}_{2} \mathrm{O}>0.6\right)$, metaluminous rocks, showing contents of $\mathrm{TiO}_{2}, \mathrm{FeO}_{\mathrm{T}}, \mathrm{MgO}, \mathrm{MnO}, \mathrm{P}_{2} \mathrm{O}_{5}, \mathrm{Cr}$ and $\mathrm{V}$ which allow them to be classified (Rios, 2002) as tholeiitic. Their REE contents are similar to low enriched MORB (Wilson, 1989) with low fractionation of LREE and nearly flat HREE patterns (Rios, 2002). They are more 
enriched in REE than the mafic rocks from the Itapicuru greenstone belt (see Silva, 1987).

Previous Nd isotopic data showed evidence of Paleoarchaean ( $T_{\mathrm{DM}}$ of $3336 \mathrm{Ma}$ ) mantle extraction ages for the oldest gneisses (Oliveira et al., 1999). Two samples from the gneiss, one from an amphibolite layer and three from intrusive granitoids were selected for this study.

\subsubsection{Uauá complex: Maracanã gneiss-sample NS 2830 (ca $3166 \mathrm{Ma}, \mathrm{ca} 2079 \mathrm{Ma}$ )}

Sample NS 2830 was mapped as gneissic-migmatitic basement of the Uauá complex (Inda et al., 1976). The sample was collected near Monte Santo city, northwestern portion of Serrinha nucleus (Fig. 2), from the "Maracanã" dimension stone quarry where the unit is well exposed. The gneissic bands are oriented $315^{\circ}-330^{\circ}$, showing steep SW to sub-vertical dips.

The zircon crystals from this sample show well-developed prisms, many of which consist of large fractured cores surrounded by clear light to pale brown overgrowths (Fig. 3A and B). Data from seven $\mathrm{U}-\mathrm{Pb}$ SHRIMP analysis are reported in Table 2 and plotted in a conventional $\mathrm{U}-\mathrm{Pb}$ concordia plot in Fig. 3C. All data are nearconcordant and plot in two groups. Three spots on overgrowth material give a weighted mean ${ }^{207} \mathrm{~Pb} /{ }^{206} \mathrm{~Pb}$ age of $2079 \pm 34 \mathrm{Ma}$, whereas 3 spots on cores cluster within error at $3025 \pm 13 \mathrm{Ma}$. The older age likely represents crystallization of a Mesoarchaean protolith, which was partially melted during the Transamazonian event.

Fragments of overgrowth that were broken off the tips of three zircon crystals from this sample and analyzed by ID-TIMS (Table 3) gave slightly discordant data that are slightly outside of error but, overlap with the SHRIMP data. Together, the Paleoproterozoic data cluster gives an Isoplot model 2 age of $2077 \pm 16 \mathrm{Ma}$. These data suggest that the time of Transamazonian fabric development was quite late and roughly synchronous with late peraluminous potassic plutonism (see below).

\subsubsection{Santa Luz complex-Valente gneiss: sample 1552 $(3102 \pm 5 \mathrm{Ma})$}

Sample NS 1552 is a peraluminous biotite-trondhjemite gneiss (Rios, 2002) from the southern part of the Serrinha nucleus (Santa Luz complex, Fig. 2), $8 \mathrm{~km}$ east of Valente city.Two zircon populations were identified in this sample. [P1] comprises metamict, honey-brown colored, prismatic zircon crystals, some with cores and overgrowths (Fig. 4A), although the cores are extremely altered. [P2] are mainly euhedral, clear, transparent zircon crystals, colorless to brown-pink, some with black inclusions. Four crystals were analyzed by TIMS (Table 3). Analyses 1-3 [P2] are discordant but give an upper intercept age of $3102 \pm 5 \mathrm{Ma}$ (Fig. 4B), which is interpreted as the minimum crystallization age of these rocks, as long as no cores were analyzed. Zircon 4 fragment, from a [P1] overgrowth, gave a concordant $2093 \pm 4$ Ma age (Table 3 ), in agreement with the age presented by Melo et al. (1995), and suggests that overgrowths of P1 developed late, probably due to the Transamazonian thermal event that accompanied emplacement of the potassic rocks.

The $\mathrm{Rb}-\mathrm{Sr}$ and $\mathrm{Sm}-\mathrm{Nd}$ isotopic ratios obtained for sample 1536, another sample of this $3102 \mathrm{Ma}$ gneiss-migmatite collected near Santa Luz City, gave $\varepsilon \mathrm{Nd}=+4.99, T_{\mathrm{DM}}=2.85 \mathrm{Ga}$, and low initial $\mathrm{Sr}$ ratio (Table 4).

\subsubsection{Amphibolite from Uauá complex-sample 1662 (metamorphic age $2078 \pm 2 \mathrm{Ma}$ )}

Sample NS 1662 was collected from an amphibolite layer in a gneiss-migmatitic unit from the northwest area of the Serrinha nucleus (Uauá complex, Fig. 2). These amphibolites have a tholeiitic

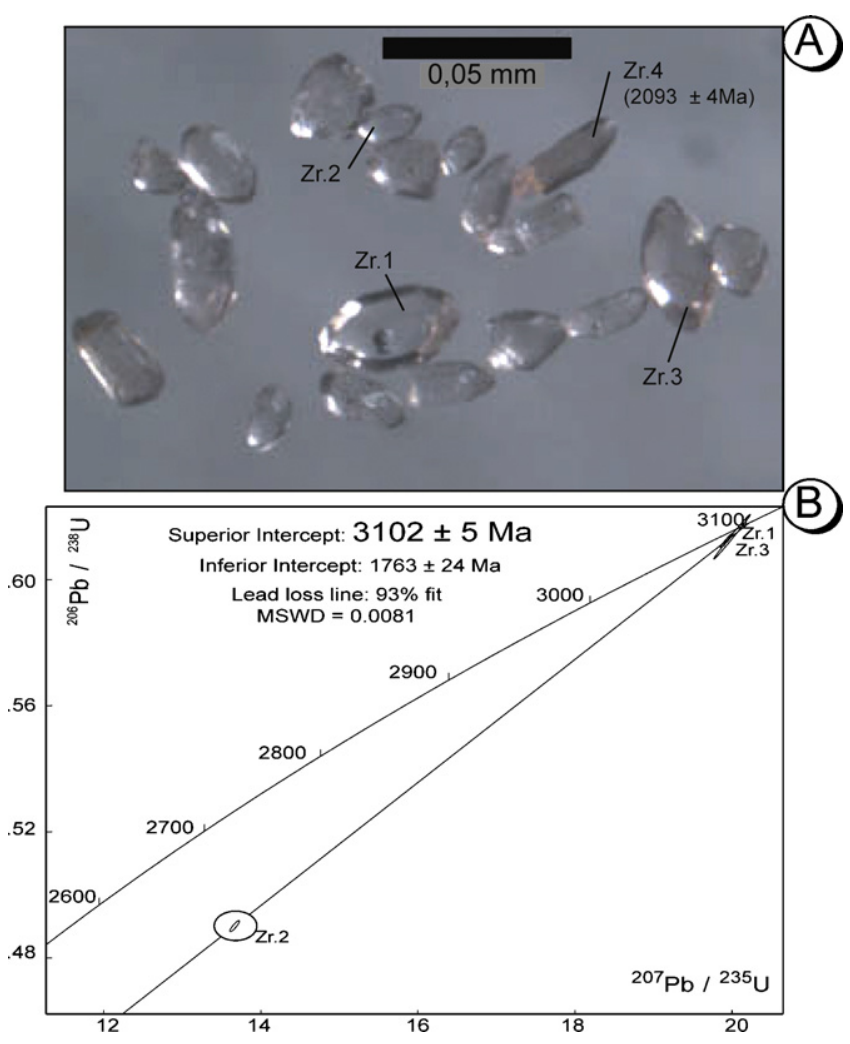

Fig. 4. Sample 1552-Biotite-Trondhjemite-Gneiss of Santa Luz complex. (A) Zircon population. (B) ID-TIMS Concordia diagram.

affinity, but it is not certain whether they were originally volcanic or intrusive.

The zircon crystals from sample 1662 are of excellent quality (Fig. 5A); large, colorless to pale pink, anhedral to subhedral, rounded, and without inclusions or fractures. Four zircon crystals were analyzed by ID-TIMS and seven by SHRIMP. The results are concordant, and TIMS data give the most precise concordia age of $2078 \pm 2 \mathrm{Ma}$ (Fig. 5B, Table 3). This may represent a magmatic age if the protolith were a gabbro that crystallized magmatic zircon. However, it is much more likely that the zircon is metamorphic in origin, since magmatic zircon is relatively rare in mafic rocks.

\subsubsection{Ambrósio pluton-sample 1351 (3162 \pm 26 Ma)}

The Ambrósio pluton forms the largest pluton in the Itapicuru greenstone belt, clearly intruding the lower volcanic sequence (Fig. 2, Matos and Davison, 1987). It was first described by Matos (1988) and later structural details have been studied by Alves da Silva et al. (1993), Chauvet et al. (1997a) and Lacerda (2004). Its rocks are considered chemically similar to the felsic volcanic (intermediate) sequence of the Itapicuru greenstone belt (Silva, 1987; Matos, 1988). Around the Ambrósio pluton is a metamorphic aureole from amphibolite $(\sim 500 \mathrm{~m})$ to greenschist facies (Alves da Silva et al., 1993). The borders of the pluton exhibit variable gneissic foliation trends with a generally sub-vertical dip which is also identified in the surrounding volcanic rocks (Matos and Davison, 1987). The core of the pluton is slightly deformed granite and granodiorite, in contrast to the border rocks, which are composed of strongly foliated and banded granite and trondhjemite. Late granitic dykes, similar to the Morro do Lopes granite ( $2070 \mathrm{Ma}$, Rios et al., 2000, 2004, see below), are found on the borders (Argolo, 1993; Alves da Silva et al., 1993; Rios, 2002; Mello et al., 2006). Amphibolite basement xenoliths, probably representative of the 

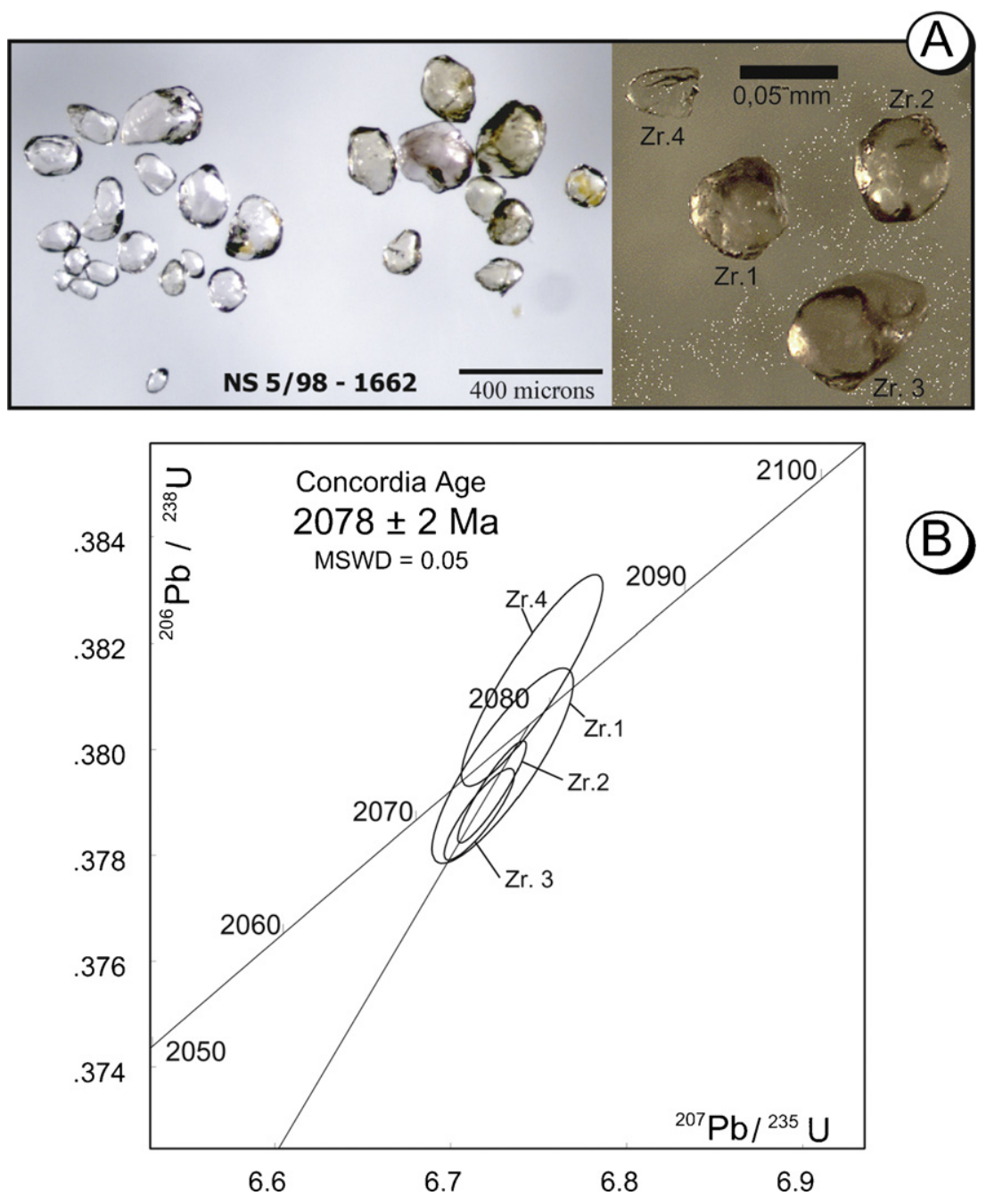

Fig. 5. Sample 1662-Amphibolite layer at Uauá complex. (A) Zircon general population. (B) ID-TIMS Concordia diagram.

mafic volcanic rocks from the Itapicuru greenstone belt, are found near the margins, with linear contacts and concordant N-S foliation.

Sample NS 1351, a peraluminous granodiorite with trondhjemitic affinity, was collected in the central-south core of the Ambrósio pluton, east of Varzea da Pedra village (Fig. 2). This sample yielded zircon crystals that are generally of poor quality (Fig. 6A). Subhedral grains are zoned with some broad zones of intense alteration. Some totally altered zircons are milky white. BSE images show variable euhedral zonation with some thin overgrowths and occasional rounded cores (Fig. 6B). Analyses were done on six single zircons by ID-TIMS and on seven different crystals by SHRIMP with the data plotted on a conventional concordia diagram (Fig. 6C) and presented in Tables 2 and 3. Most ID-TIMS analysis show severe $\mathrm{Pb}$ loss, but two near-concordant data yield different ages of $3079 \pm 9 \mathrm{Ma}$ and $3169 \pm 2 \mathrm{Ma}$. The younger age is from a fragment of overgrowth that showed unusually low U concentration. The seven SHRIMP analyzed zircon grains generally show magmatic Th/U $(>0.39)$ ratios. The six most concordant SHRIMP data have ${ }^{207} \mathrm{~Pb} /{ }^{206} \mathrm{~Pb}$ ages that overlap within error and give an average age of $3162 \pm 26 \mathrm{Ma}(\mathrm{MSWD}=1.3)$, which agrees with the older TIMS analysis (3169 Ma) and is interpreted as the magmatic age of the Ambrósio pluton. Most SHRIMP analysis were carried out on broadly zoned interiors of crystals to avoid alteration. Attempts to date overgrowths resulted in discordant data. One Palaeopro- terozoic age (9.1) on a high U overgrowth showed very low Th/U (0.01), suggesting that the sample was metamorphosed during the Transamazonian event.

\subsubsection{Araci pluton-samples 1599 and 1602 (3072 $\pm 2 \mathrm{Ma}$ and $2060 \mathrm{Ma})$}

The Araci pluton, located at the southeastern border of the Serrinha nucleus (Fig. 2), was first described as a pretectonic body (Matos and Conceição, 1993; Rios et al., 1998). Its western side is in contact with mafic and sedimentary units of the Itapicuru greenstone belt and on the east, it is partially covered by Mesozoic sediments of the Tucano-Reconcavo basin. Most of the contacts are marked by faults.

Sample NS 1599, a monzo-granite, and NS 1602, a syeno-granite, were collected on the northern border of the Araci pluton (Fig. 2) Zircon from these samples shows considerable complexity, including the presence of cores and multiple stages of growth, as well as considerable alteration (Fig. 7A and B).

Three single zircon crystals from NS1599 were selected for IDTIMS analysis. Zircon 1 ( $2911 \pm 103 \mathrm{Ma})$ showed the least discordant (5\%) result, although there is a high error associated with this analysis and its low $\mathrm{Th} / \mathrm{U}$ ratio (0.08) suggests that it is of metamorphic origin. The other two TIMS analysis are also Archean but quite discordant, defining an upper concordia intercept age of $3072 \pm 2 \mathrm{Ma}$ (Fig. 7C). 

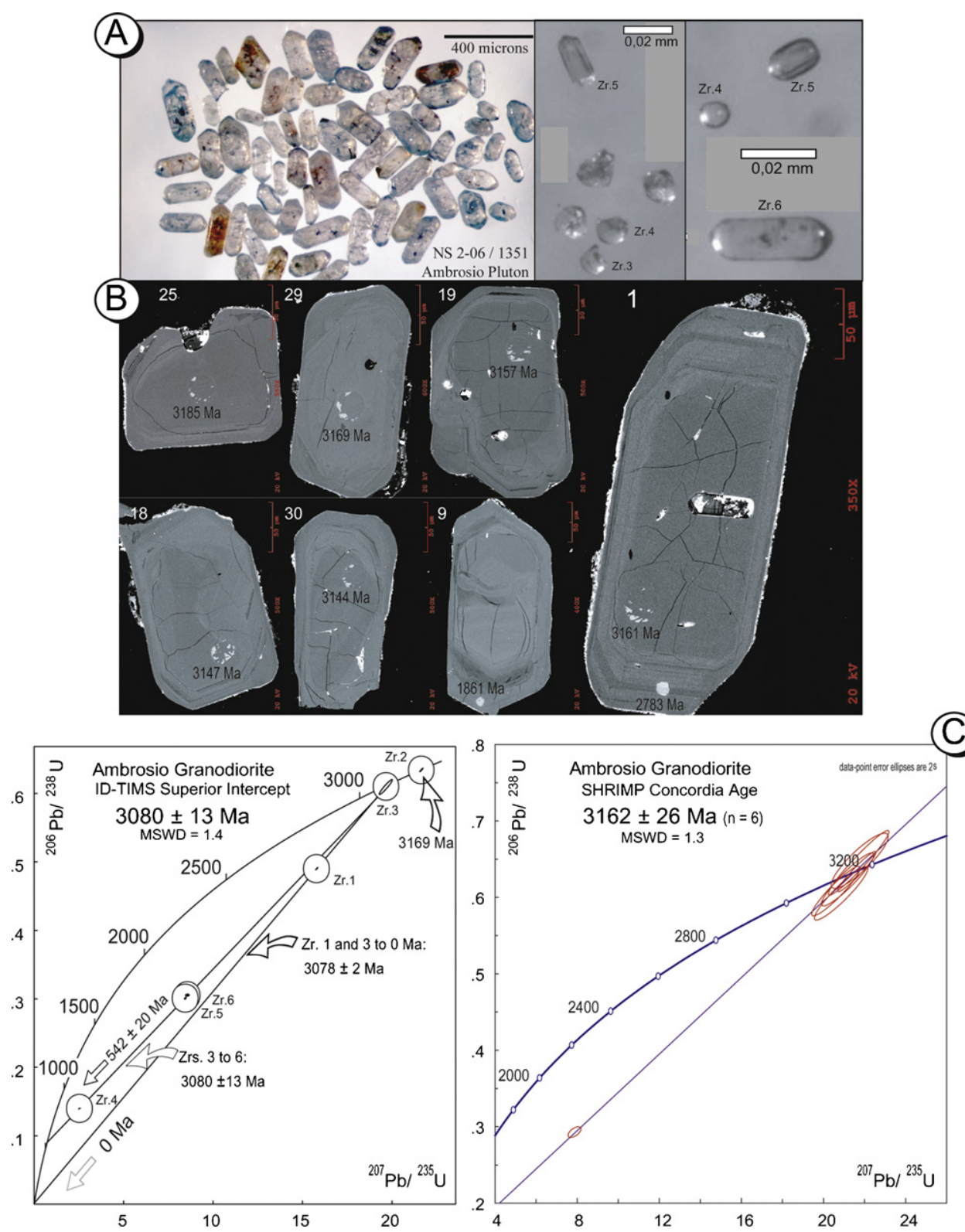

Fig. 6. Sample 1351-Ambrósio Granodiorite. (A) Zircon population. (B) BSE images of SHRIMP analyzed crystals. (C) ID-TIMS and SHRIMP Concordia diagrams.

Only unzoned cores have sufficiently large unaltered domains to be analyzed by SHRIMP (Fig. 7B). Three analysis (2 from sample 1602 and 1 from sample 1599) overlap on concordia giving an age of $2059 \pm 8 \mathrm{Ma}$ (Fig. 7C). Two of these grains show low Th/U ratios ( 0.07 and 0.15 ), whereas the third is unusually high $(\mathrm{Th} / \mathrm{U}=1.32$, Table 2) for magmatic zircon (1.3). These data might indicate: (i) that the Archaean zircon analyzed by TIMS is xenocrystic; (ii) that the Araci pluton represents a late Transamazonian phase; or (iii) that the zircon analyzed by SHRIMP was derived from late veins within the sample, or are metamorphic in origin.

The $\mathrm{Rb}-\mathrm{Sr}$ and $\mathrm{Sm}-\mathrm{Nd}$ isotope ratios obtained for sample 1599 (at $3070 \mathrm{Ma}$ ) result in $\varepsilon \mathrm{Nd}=+0,93, T_{\mathrm{DM}}=3.12 \mathrm{Ga}$, although the initial ${ }^{87} \mathrm{Sr} /{ }^{86} \mathrm{Sr}$ of 0.6302 is unreliable and probably reflects disturbance of this isotopic system.

\subsubsection{Requeijão pluton-sample $1555(2989 \pm 11 \mathrm{Ma})$}

The Requeijão pluton was identified during a regional mapping project (Melo et al., 1995) and described as belonging to the intru- sive suite of Palaeoproterozoic rocks. Later, Matos and Conceição (1993) and Rios et al. (1998) re-classified this pluton, interpreting it as one of the pretectonic granites of the Serrinha nucleus.

The Requeijão pluton is located in the southwestern area of the Serrinha nucleus, near the boundary with the Salvador-Curaçá mobile belt (Fig. 2). Sample NS 1555 is a biotite-granite and it was collected on the north border zone. Most of the zircon crystals are subhedral to euhedral, show overgrowths, and are highly metamict (Fig. 8A). Typically, BSE images show fine oscillatory zoning with thin unzoned overgrowths. Most grains are strongly affected by cracking and alteration (Fig. 8B). ID-TIMS analysis of a fraction of five small crystals (Zr.1), three overgrowths (Zr. 2-4) and one subhedral non-abraded grain (Zr. 5) gave complex results (Table 3). All of the grains are highly discordant (19-44\%, Fig. 8C), giving an upper intercept age of 2870 Ma. The accuracy of this result is unclear because of the discordance but it is consistent with a Mesoarchean age for the Requeijão pluton. 

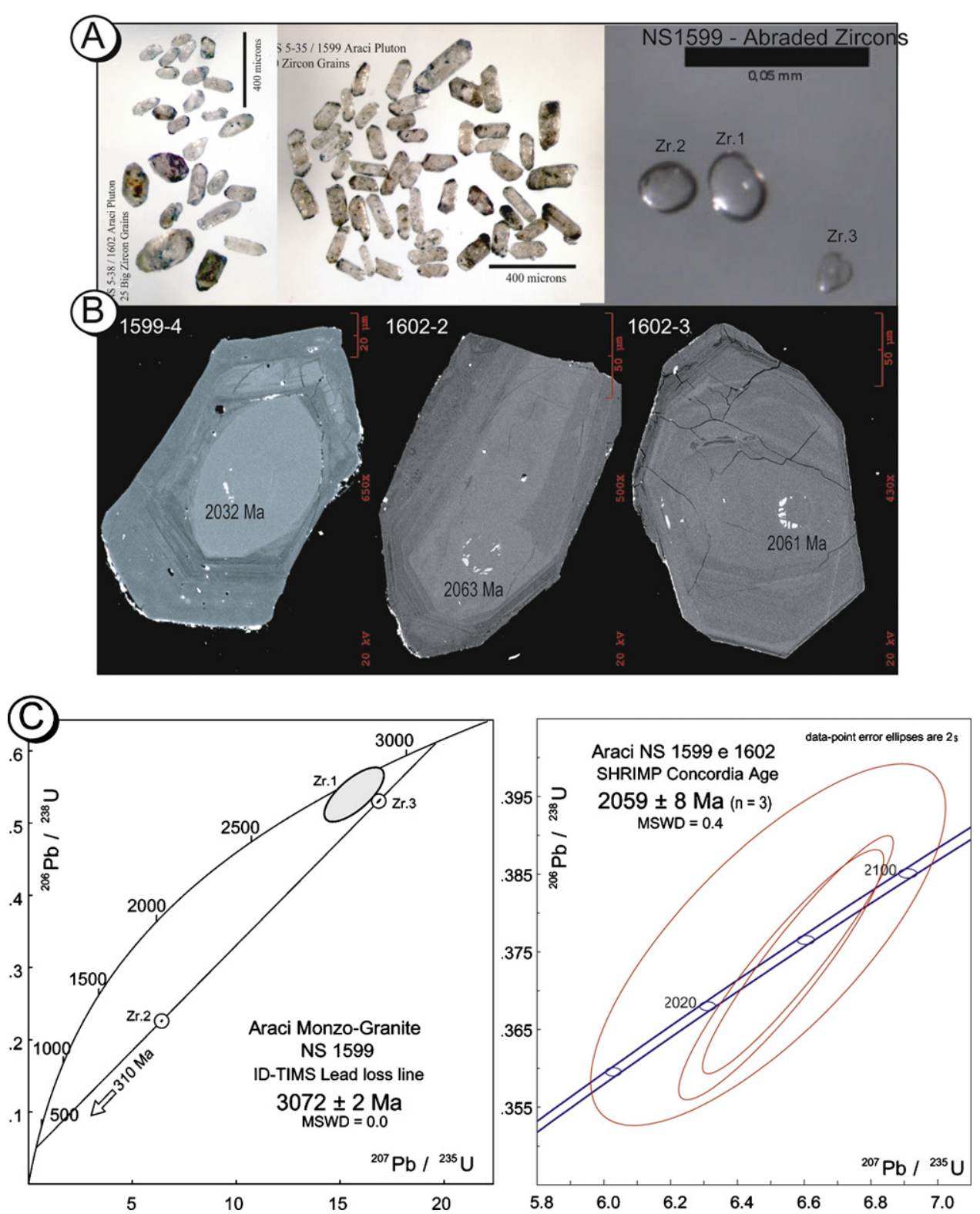

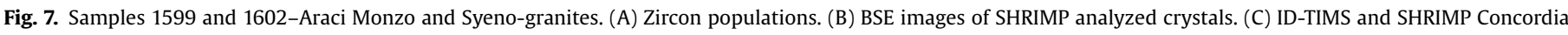
diagrams.

As with TIMS, many SHRIMP U-Pb analysis of zoned zircon domains from sample NS 1555 show lead loss (Fig. 8C), but three analysis overlap on, or near, concordia, giving an average ${ }^{207} \mathrm{~Pb} /{ }^{206} \mathrm{~Pb}$ age of $2989 \pm 11 \mathrm{Ma}$ (MSWD of 0.89). This is interpreted to represent the age of crystallization of this pluton.

\subsection{Pretectonic Palaeoproterozoic granitoid rocks}

We present new geochronological data for two pretectonic TTG plutons; the Eficéas and Cipó plutons.

\subsubsection{Eficéas pluton-sample $1399(2163 \pm 5 \mathrm{Ma})$}

Sample NS 1399 is a biotite-trondhjemite from the central area of Eficéas pluton (Fig. 2). The zircon population is represented by small, subhedral to euhedral, elongated, brownish to colourless crystals, some with black and melt inclusions (Fig. 9A). Most are metamict and fractured. Two colourless grains gave relatively concordant data and, together with a more discordant (18\%) brownish grain with a cloudy interior, define an ID-TIMS lead loss line with an upper intercept age of $2163 \pm 5 \mathrm{Ma}$ (Fig. 9C, Table 3). The lower intercept age is of $443 \pm 30$ Ma.

Many grains show unzoned euhedral interiors with zoned overgrowths under BSE (Fig. 9B). Three out of four SHRIMP analysis overlap within error of concordia (Fig. 9C) and define an average ${ }^{207} \mathrm{~Pb} /{ }^{206} \mathrm{~Pb}$ age of $2157 \pm 23 \mathrm{Ma}(\mathrm{MSWD}=1.1)$. This is consistent with the 2163 Ma TIMS age, which we interpret as the crystallization age for the Eficéas pluton. The Sm-Nd isotope ratio obtained for sample NS 1399 (at $2163 \mathrm{Ma}$ ) gives $\varepsilon \mathrm{Nd}=+1.64, T_{\mathrm{DM}}=2.33 \mathrm{Ga}$.

\subsubsection{Cipó pluton: sample NS $1543(2164 \pm 2 \mathrm{Ma})$}

The Cipó pluton is located near the western boundary of the Serrinha nucleus, almost $5 \mathrm{~km}$ from the Salvador-Curaçá mobile belt (Fig. 2). Matos and Conceição (1993) classified it as a late tectonic granite (Morro do Lopes type).

Sample NS 1543 is a peraluminous granite. Its zircon population is composed of translucent to transparent crystals, honey-coloured 

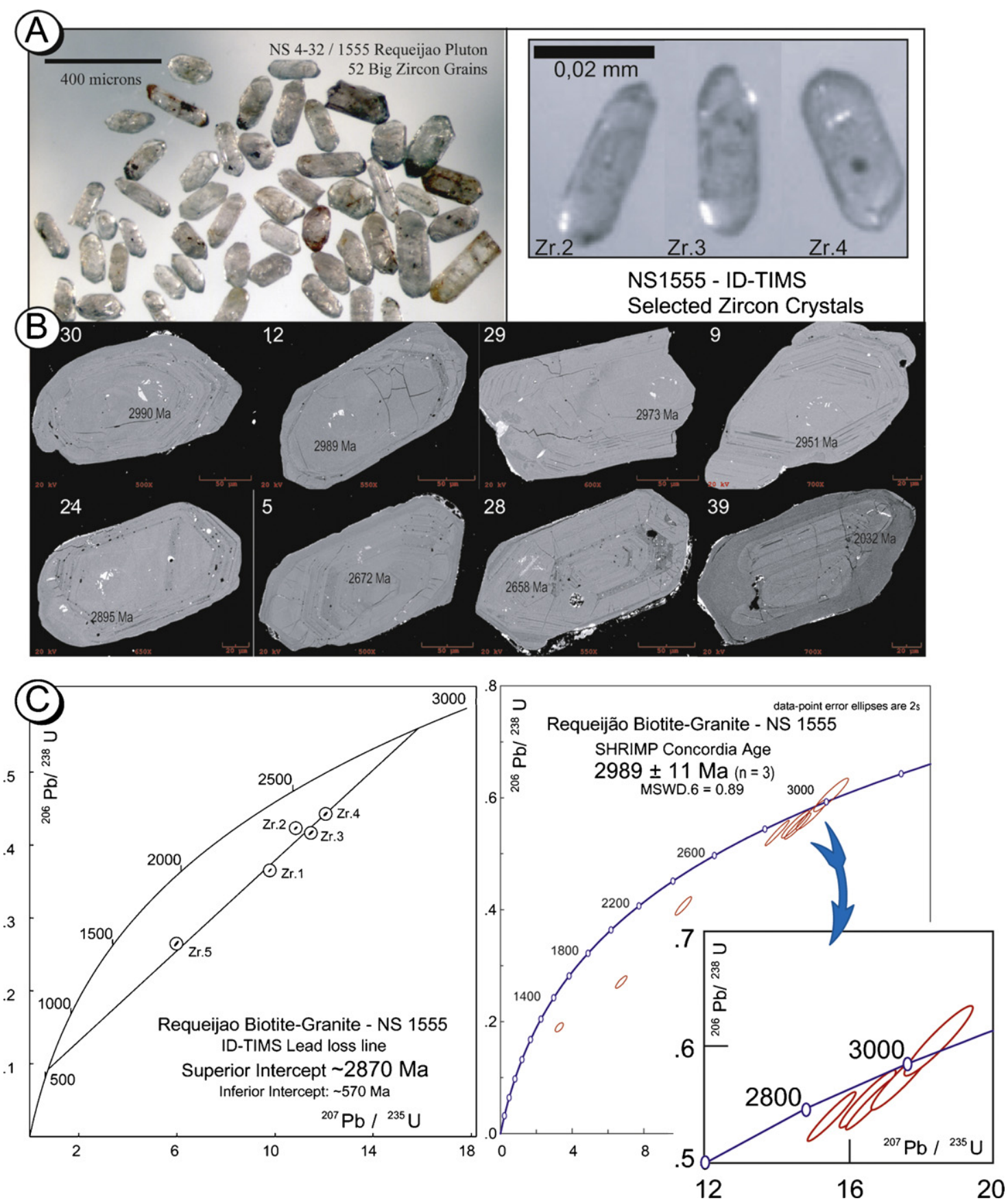

Fig. 8. Sample 1555-Requeijão Biotite-Granite. (A) Zircon population. (B) BSE images of SHRIMP analyzed crystals. (C) ID-TIMS and SHRIMP Concordia diagrams.

to colourless, rounded and elongated, subhedral to euhedral, showing zoning typical of magmatic grains, some with black and yellow melt inclusions. Most crystals are altered and metamict, fractured, and rounded (Fig. 10A and B). BSE images show that the zircon grains generally have unzoned euhedral interiors surrounded by zoned overgrowths (Fig. 10B), as with the Eficéas pluton above. Rios et al. (1998) reported a Pb-Pb (Kober methodology) zircon age of $2050 \pm 51 \mathrm{Ma}$ for this sample. U-Pb TIMS analysis were performed on four grains. Regressing three data (from rounded colourless grains), one of which is concordant, gives an upper intercept age of $2164 \pm 2$ Ma with a lower intercept of $886 \pm 160 \mathrm{Ma}$. One pink to brown elongated, euhedral crystal (Zr. 4) gave a concordant datum at $2088 \pm 3 \mathrm{Ma}$ (Fig. 10C). Six out of seven SHRIMP data are within error of concordia (Fig. 10C), but appear to fall into two clusters. The older cluster gives an average ${ }^{207} \mathrm{~Pb} /{ }^{206} \mathrm{~Pb}$ age of $2170 \pm 16 \mathrm{Ma}$ (MSWD $=1.14$ ), whereas the younger cluster is $2100 \pm 11 \mathrm{Ma}$ $(\mathrm{MSWD}=0.74)$. Most of these analysis were done on unzoned interiors of grains.

Zircon from the Cipó pluton appears to have a bimodal age distribution, as shown by both SHRIMP and TIMS. Th/U ratios from all analysis fall within the range for magmatic zircon. In view of this and the peraluminous composition of the pluton, there are two possibilities: (i) the crystallization age of the Cipó pluton is 2164 Ma and there was new zircon generation related to the $\sim 2.07$ Ga metamorphic event, or (ii) that this pluton represents a melt of sedimentary rocks derived from pretectonic $(2.15 \mathrm{Ga})$ and possibly syntectonic $(2.1 \mathrm{Ga})$ plutons. In that case, its crystallization age would probably 

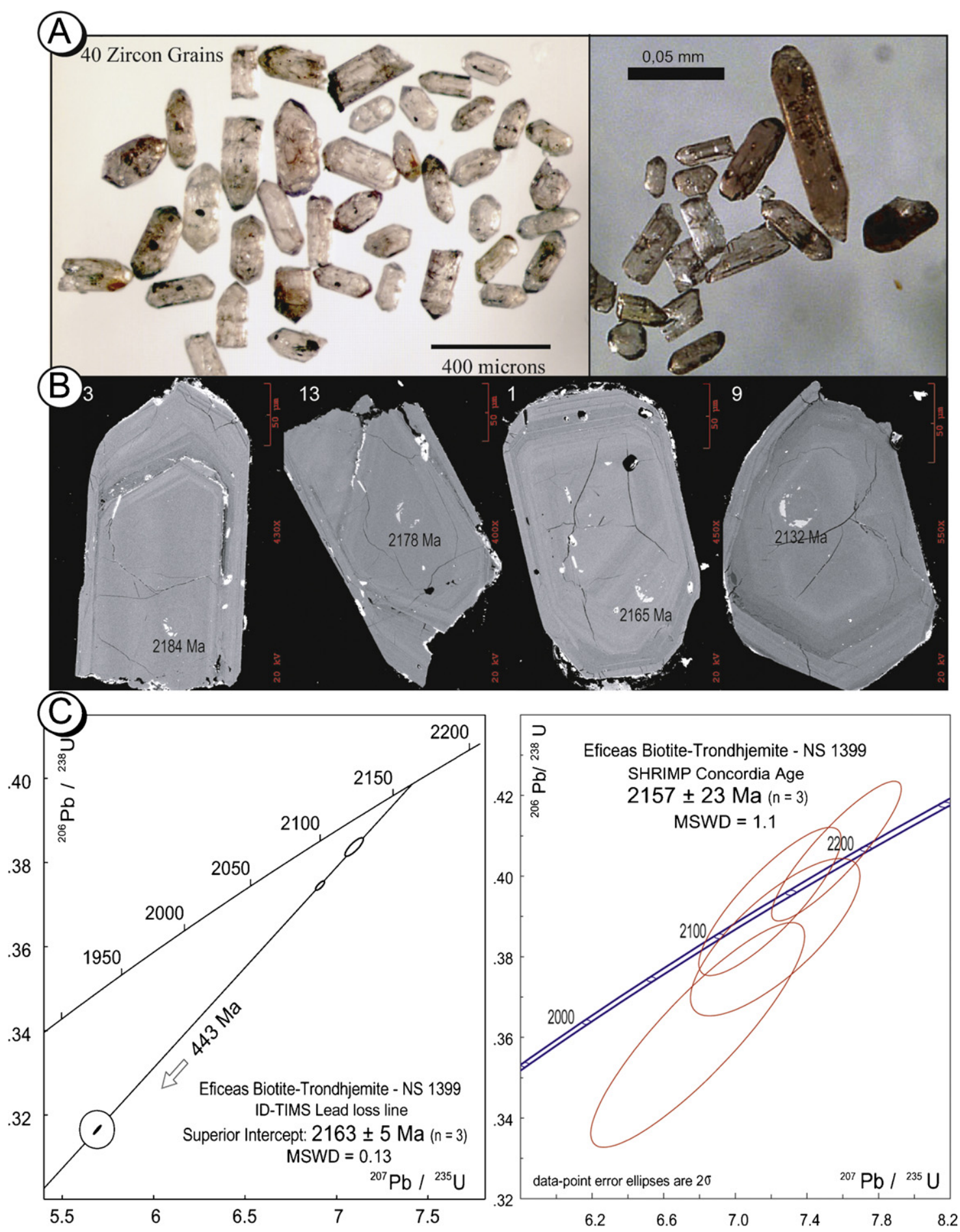

Fig. 9. Sample 1399-Eficéas Biotite-Trondhjemite. (A) Zircon population. (B) BSE images of SHRIMP analyzed crystals. (C) ID-TIMS and SHRIMP Concordia diagrams.

be close to the age of the youngest concordant datum, shown by TIMS to be $2088 \pm 3 \mathrm{Ma}$.

\subsection{Late-tectonic Palaeoproterozoic granitoid rocks}

\subsubsection{Itareru diorite: sample NS $1358(2106 \pm 2 \mathrm{Ma})$}

Zircon from sample NS 1538, a diorite from the central region of the Itareru shoshonitic pluton (Fig. 2), is composed of pink to brown, irregular shaped crystals, subhedral to euhedral, some with black inclusions and overgrowths (Fig. 11A). Two distinct populations have been identified. [P1] is represented by clear, transparent zircons, which tend to be rounded. [P2] zircon has altered cores with clear and transparent overgrowths. Four crystals were selected for ID-TIMS analysis. Zrs. 1 and 2 are overgrowths manually broken from the P2 grains. Zr. 3, with an altered core, comes from P2. Zr. 4 is one of the clear zircons from P1. Zircon crystals from this sample have high $U$ contents (Table 3). Zircons 1, 2 and 4 gave discordant data that can be regressed with an upper intercept age of $2106 \pm 2 \mathrm{Ma}$, which is interpreted as the crystallization age of this pluton (Fig. 11C). Zr. 3 gave a slightly discordant 

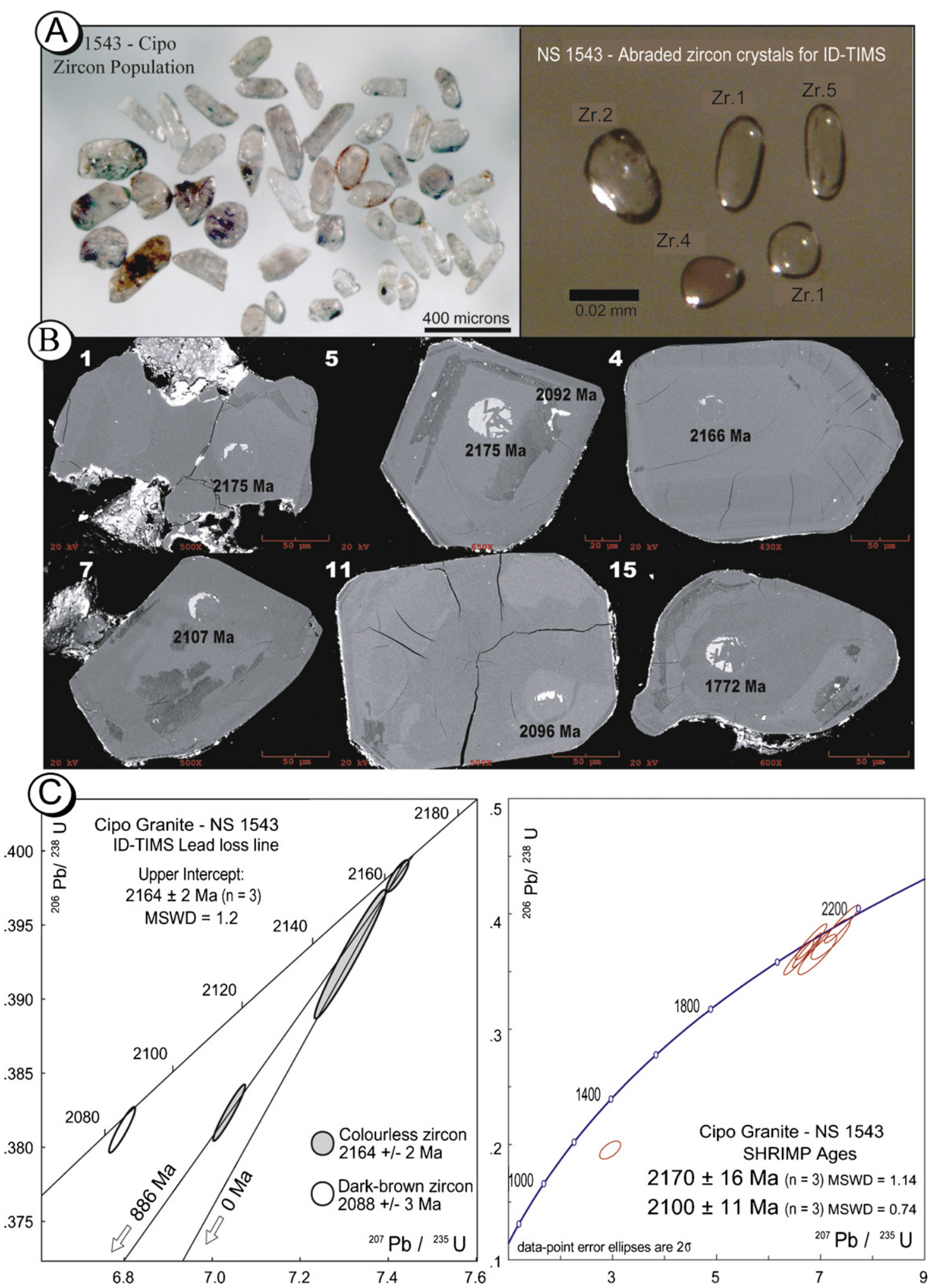

Fig. 10. Sample 1543-Cipó Granite. (A) Zircon population. (B) BSE images of SHRIMP analyzed crystals. (C) ID-TIMS and SHRIMP Concordia diagrams.

datum with a ${ }^{207} \mathrm{~Pb} /{ }^{206} \mathrm{~Pb}$ age of $2162 \pm 4 \mathrm{Ma}$ and is interpreted as a xenocryst.

\subsubsection{Itareru lamprophyre: sample NS 2595 (2113 $\pm 5 \mathrm{Ma})$}

Sample 2595 is from a lamprophyric dyke within the Itareru pluton, near the contact with gneisses of the Santa Luz complex (Fig. 2).
Zircon crystals from this sample are large, euhedral, prismatic, brown, translucid and most show internal cloudy areas, zoning and possible cores (Fig. 12A). However, BSE images show little evidence of cores, most grains having euhedral zoning occasionally with thin overgrowths (Fig. 12B). Eight analysis on seven grains produced overlapping concordant data with an average ${ }^{207} \mathrm{~Pb} /{ }^{206} \mathrm{~Pb}$ 


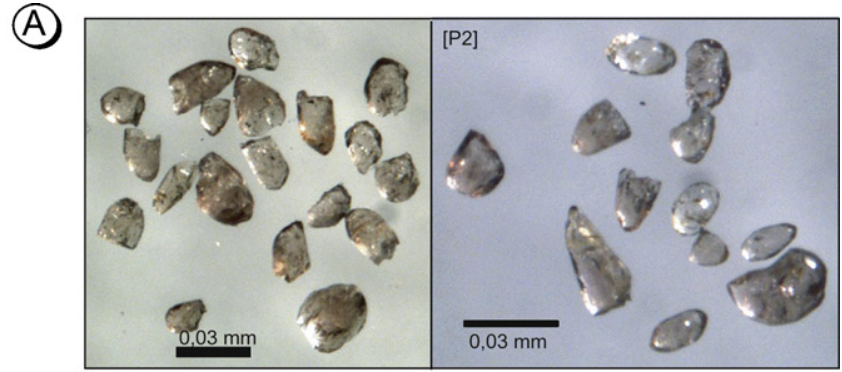

(B)

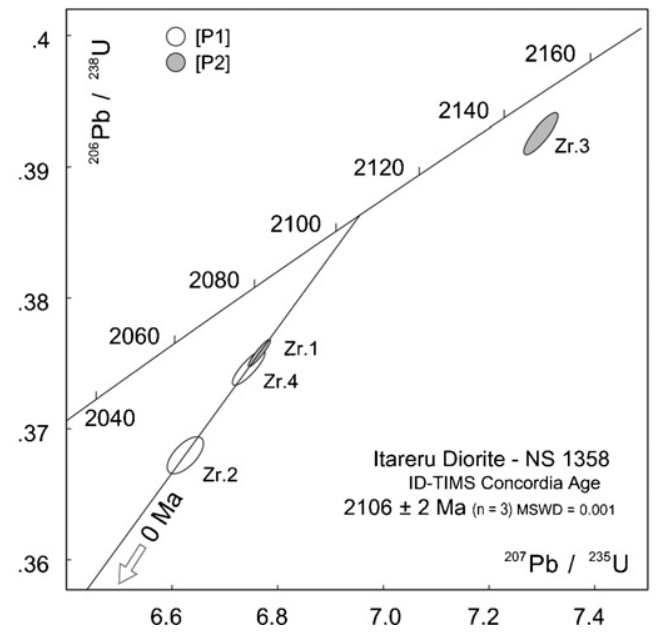

Fig. 11. Sample 1358-Itareru Diorite. (A) Zircon populations. (B) ID-TIMS Concordia diagram.

age of $2113 \pm 5 \mathrm{Ma}$, which overlaps with the age of the Itareru diorite (sample 1358).

\subsubsection{Pedra Vermelha pluton: sample NS 1664 (2080 \pm 8 Ma)}

The Pedra Vermelha is a circular pluton located in the northwestern area of the Serrinha nucleus, where it intrudes metabasites related to the Uauá complex (Fig. 2, Inda et al., 1976). It is considered to be related to the pretectonic granites of Matos and Conceição (1993).

Sample 1664 is a biotite-granite. Two distinct zircon populations have been identified: [P1] is represented by altered crystals, metamict and rounded, which could not be analyzed. [P2] consists of small, pale pink to colorless, clear and shiny, subhedral zircon crystals (Fig. 13A). One concordant datum and three variably discordant data from the least cracked grains define a $\mathrm{Pb}$ loss line with an upper intercept age of $2080 \pm 8 \mathrm{Ma}$ (Fig. 13B, Table 3). This is likely the age of crystallization of the pluton.

\subsubsection{Barroquinhas pluton: sample NS 1587 (2073 $\pm 1 \mathrm{Ma}$ )}

The Barroquinhas pluton is a small, slightly E-W elongated pluton, intruding gneisses and basalts of the Santa Luz complex and the Itapicuru greenstone belt, respectively (Alves da Silva et al., 1993; Chauvet et al., 1997a). It is located near the southeast limit of Itapicuru rocks, only a few kilometers from the Mina Brasileiro gold mine (Fig. 2).

Sample 1587 is a biotite-granite which yielded two distinct zircon populations: [P1] are metamict, very altered, rounded crystals, which could not be analyzed: [P2] are subhedral to euhedral, pale pink to honey-brown, clear crystals of variable size, some with fractures (Fig. 14A). No core-overgrowth relationships were identified. Five of the best grains were selected for analysis and give data that range from concordant to mildly discordant (Fig. 14B).
A regression line gives an upper intercept ${ }^{207} \mathrm{~Pb} /{ }^{206} \mathrm{~Pb}$ age of $2073 \pm 1 \mathrm{Ma}$.

\subsubsection{Fazenda Bananas pluton: sample NS 1436 (2072 $\pm 1 \mathrm{Ma})$}

Sample 1436 is a biotite-monzogranite from a small stock that intrudes the Agulhas-Bananas syenite. It shows two distinct zircon populations: [P1], the most abundant population, is composed of small pink to colorless, clear, transparent, euhedral to subhedral zircon crystals (P class of Pupin, 1980): [P2] consists of rare dark brown, subhedral to anhedral, rounded crystals, some with inclusions and zoning.

Four grains were analyzed: two (Zr. 2 and 3) from [P1], and two (Zr. 1 and 4) from [P2]. Grains from different populations produced discordant data but these do not appear to have significant age differences. Two slightly discordant data give overlapping ${ }^{207} \mathrm{~Pb} /{ }^{206} \mathrm{~Pb}$ ages with an average of $2072 \pm 1 \mathrm{Ma}$ (Fig. 15A). Regression of these with the most discordant datum give a similar upper intercept age of $2074 \pm 2$ Ma (Fig. 15B).

Table 4 gives $\mathrm{Rb}-\mathrm{Sr}$ and $\mathrm{Sm}-\mathrm{Nd}$ data for three samples from the Morro do Lopes type granites. The initial $\mathrm{Sr}$ and $\mathrm{Nd}$ ratios of these rocks are within narrow ranges, 0.688217-0.7029 and $0.509415-0.509748$, respectively. $\varepsilon_{\mathrm{Nd}}(T)$ values range from -4.07 to -10.54 (Table 4 ). Nd $T_{\text {DM }}$ model ages are 2.67 to $3.10 \mathrm{Ga}$, assuming a two-stage evolution as proposed by De Paolo and Schubert (1991). These results differ significantly from the ones shown by syenite and shoshonite of the same group (Table 4).

\section{Discussion}

\subsection{Stages of granitoid plutonism}

Based on the data presented here (see summary in Table 5), we define three major groups of plutonic rocks exposed in the Serrinha nucleus:

Group 1: This is the oldest group and consists of ca. $3.2-2.9 \mathrm{Ga}$ Mesoarchaean migmatite, gneisses and TTG plutons that occur in the Santa Luz and Uauá complexes, and within the Itapicuru greenstone belt. Ages are scattered in some samples apparently due to the presence of Mesoarchaean remobilization and metamorphism. Zircon crystals with low Th/U metamorphic ratios confirm the minimum age of $3.07 \mathrm{Ga}$ proposed by Paixão et al. (1995) for the granulite facies metamorphism.

Group 2: The next major pulse of magmatism was the Palaeoproterozoic emplacement of calc-alkaline and TTG plutons. In this group we include the plutons of Eficéas (2163 Ma), Lagoa dos Bois, and Cipó (2164 Ma), as well as the previously dated plutons of Nordestina (2155 Ma), Quijingue (2155 Ma), Trilhado (2155 Ma), Teofilândia (2130 Ma), and Barrocas (2128 Ma) (Fig. 2, see Table 1 for references). These allow us to limit the emplacement of TTG Palaeoproterozoic magmas into the Serrinha nucleus to a period of approximately $30 \mathrm{~m} . \mathrm{y}$. (2128-2164 Ma), and to note that the younger bodies are limited to the southernmost area of the Itapicuru belt. The age of the Cipó pluton (zircon at 2164, 2100 and $2088 \mathrm{Ma}$ ) is still unclear. Matos and Conceição (1993) describe these rocks as belonging to Morro do Lopes ( $\sim 2.07-2.08 \mathrm{Ga}$, group 3) magmatism; Rios et al. (1998), however, showed Pb-Pb Kober ages from the Cipó pluton ranging from 2004 to $2165 \mathrm{Ma}$ (the best analysis is the older). The Nordestina batholith ( $2.15 \mathrm{Ga})$ also contains zircon crystals $2088 \mathrm{Ma}$ and younger (Rios et al., 1998), but clearly belongs to group 2 granitoids.

Group 3: These represent undeformed, late to post-tectonic, alkaline Palaeoproterozoic intrusions. Group 2 plutons were affected by a major period of deformation before the emplacement of 


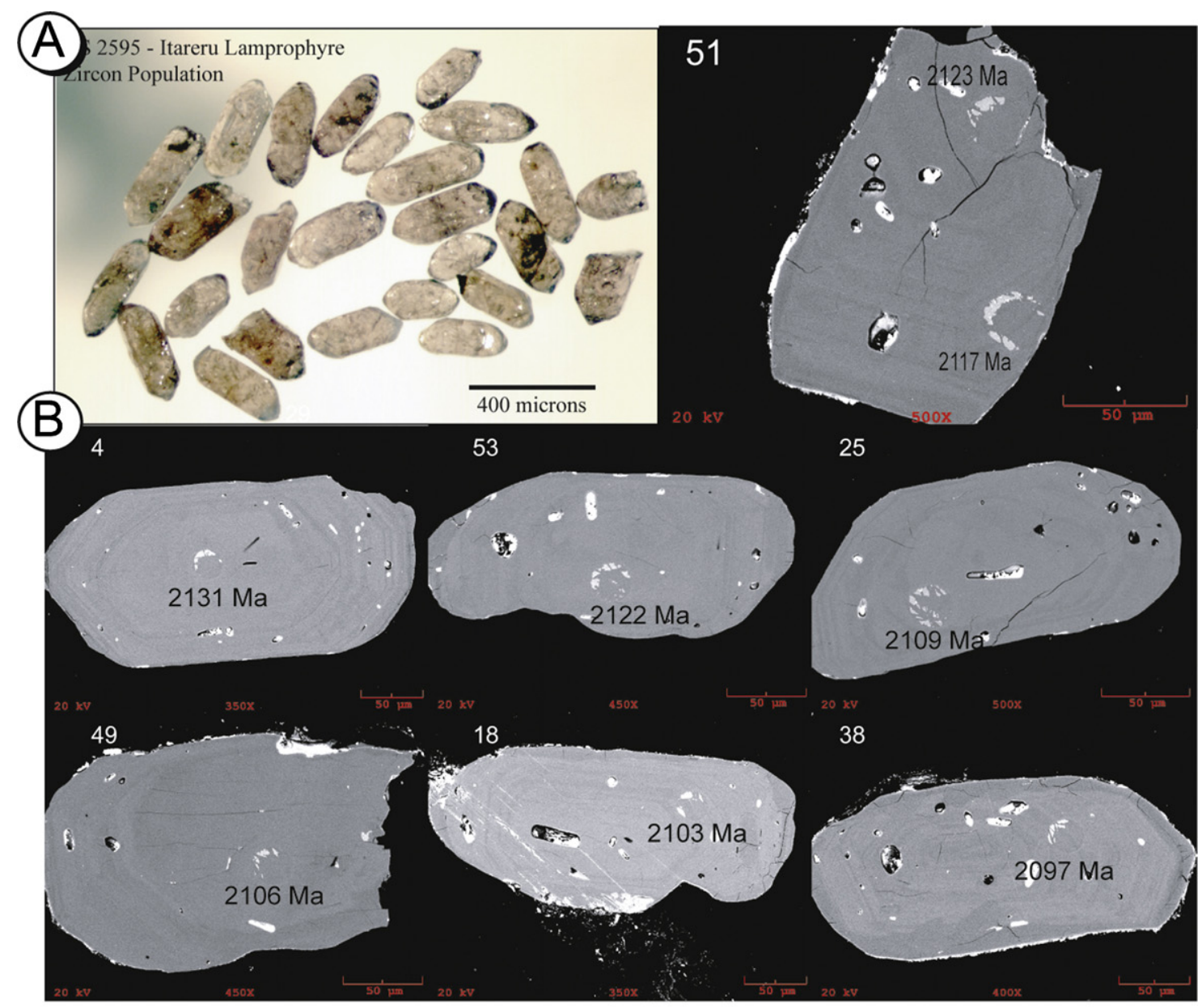

(C)

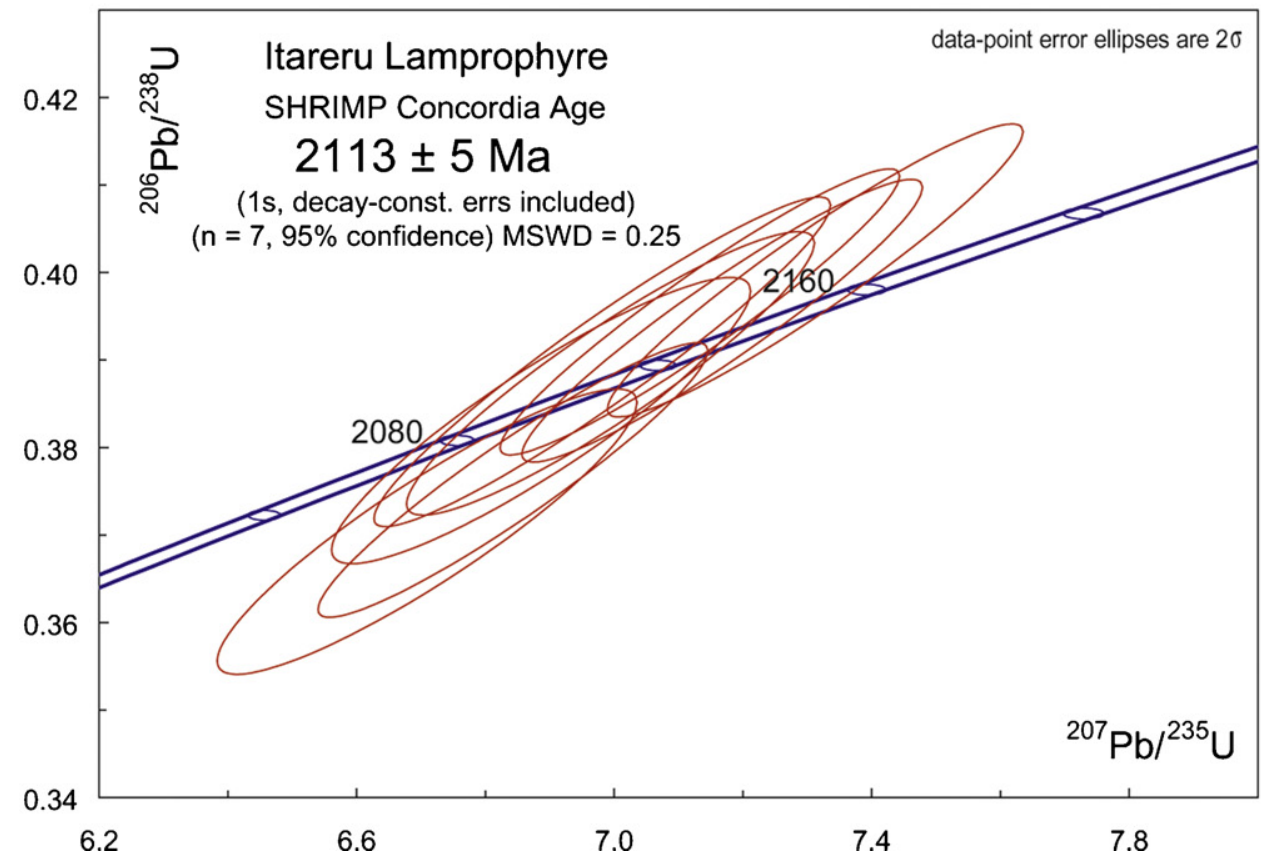

Fig. 12. Sample 2595-Itareru Lamprophyre. (A) Zircon population. (B) BSE images of SHRIMP analyzed crystals. (C) SHRIMP Concordia diagram. 
Table 5

Geochronological summary of U-Pb available data for Serrinha nucleus rocks. Data are from zircon, unless otherwise specified. $\mathrm{Zr}=$ multi-grain zircon, $\mathrm{Mz}=$ monazite, $\mathrm{Ti}=\mathrm{titanite}, \mathrm{X}=\mathrm{xenotime}, \mathrm{S}=$ sedimentation. When nothing else is specified, $\mathrm{U}-\mathrm{Pb}$ analysis are for single zircon crystals.

\begin{tabular}{|c|c|c|c|c|c|}
\hline Pluton/Lithology & Crystallization age (Ma) & Metamorphic age $(\mathrm{Ma})$ & Inherited age (Ma) & Method & Reference \\
\hline \multicolumn{6}{|l|}{$\begin{array}{l}\text { Basement units: Uauá complex } \\
\text { (UC) and Santa Luz complex } \\
\text { (SLC) }\end{array}$} \\
\hline Quijingue Gneiss-Migmatite (UC) & $3195 \pm 11$ & 3090 & $3314 \pm 20$ & SHRIMP & Rios et al. (2008) \\
\hline Maracanã Gneiss-Migmatite (UC) & ca. 3166 & $2079 \pm 34$ & & SHRIMP & This work \\
\hline Caldeirao Belt Orthogneiss (UC) & $3152 \pm 5$ & & & SHRIMP & Oliveira et al. (2002) \\
\hline Trondhjemite-Gneiss Valente (SLC) & $3102 \pm 5$ & $2093 \pm 4$ & & ID-TIMS & This work \\
\hline Retirolândia TTG (SLC) & 3085 & & & SHRIMP & Cordani et al. (1999) \\
\hline Granodioritic Ortho-Gneiss (UC) & $2991 \pm 22$ to $3152 \pm 5$ & & & SHRIMP & Mello et al. (2006), Oliveira et al. (2002) \\
\hline Gneiss TTG (UC) & $2956 \pm 39$ to $3070 \pm 3$ & $2077 \pm 11$ & & ID-TIMS & Oliveira et al. (2000) \\
\hline Enderbite Granulite (UC) & $2933 \pm 3$ & & & ID-TIMS & Oliveira et al. (2002) \\
\hline Toleitic Dykes (UC) & $2778 \pm 7$ & $2039 \pm 2(\mathrm{Ti})$ & & ID-TIMS & Oliveira et al. (2000) \\
\hline Caldeirao Belt Quartzite (UC) & $2687 \pm 16(\mathrm{E})$ & $2076 \pm 10$ & $3051 \pm 13$ to $3204 \pm 9$ & SHRIMP & Mello et al. (2006), Oliveira et al. (2002) \\
\hline Amphibolite Dykes (UC) & & $2078 \pm 2$ & & ID-TIMS & This work \\
\hline \multicolumn{6}{|l|}{ Group 1 granitoids } \\
\hline Ambrósio Granodiorite & $3162 \pm 26$ & & & ID-TIMS/SHRIMP & This work \\
\hline Rio Capim Tonalite & $3130-3050$ & 2050 & & SHRIMP & Cordani et al. (1999) \\
\hline Araci Granite & $3072 \pm 2$ & 2060 & & ID-TIMS/SHRIMP & This work \\
\hline Requeijão Biotite-Granite & $2989 \pm 11$ & & & ID-TIMS/SHRIMP & This work \\
\hline Ambrósio Gneiss & $2930 \pm 32(\mathrm{Zr})$ & & & ID-TIMS & Gaal et al. (1987) \\
\hline Pedra Alta TTG & 2650 to 3000 & & & ICP-MS-LA & Batista et al. (1998) \\
\hline Poco Grande & $2645 \pm 70$ & $2079 \pm 47(\mathrm{Mz})$ & & ID-TIMS & Melo et al., 1995 \\
\hline Ambrósio Gneissic Enclave & & & $3094 \pm 21$ to $3159 \pm 18$ & SHRIMP & Mello et al. (2006) \\
\hline Ambrósio Massive Granodiorite & $2077 \pm 22$ & & $2937 \pm 16$ to $3162 \pm 13$ & SHRIMP & Mello et al. (2006) \\
\hline Ambrósio Porphyritic Granodiorite & $2063 \pm 55$ & & $2975 \pm 7$ to $3126 \pm 5$ & SHRIMP & Mello et al. (2006) \\
\hline \multicolumn{6}{|l|}{ Greenstone belts } \\
\hline Leucogabbre Rio Capim & $2143 \pm 21$ & & & ID-TIMS & Oliveira et al. (1998) \\
\hline Diorite Rio Capim & $2148 \pm 23$ & & & ID-TIMS & Oliveira et al. (1998) \\
\hline \multicolumn{6}{|l|}{ Group 2 granitoids } \\
\hline Cipó Biotite-Trondhjemite & $2164 \pm 2$ & $2088 \pm 3$ & & ID-TIMS/SHRIMP & This work \\
\hline Eficéas Biotite-Trondhjemite & $2163 \pm 5$ & & & ID-TIMS/SHRIMP & This work \\
\hline Quijingue Trondhjemite & $2155 \pm 3$ & & $3614-3620$ & ID-TIMS/SHRIMP & Rios et al. (2008) \\
\hline Trilhado Undeformed Granodiorite & $2155 \pm 9$ & & & SHRIMP & Mello et al. (2006) \\
\hline Teofilandia Tonalite to Granodiorite & $2130 \pm 7$ & $2071 \pm 10$ & & SHRIMP & Mello et al. (2006) \\
\hline Lagoa dos Bois Granodiorite & $2107 \pm 23(\mathrm{Zr})$ & & & ID-TIMS & Gaal et al. (1987) \\
\hline \multicolumn{6}{|l|}{ Group 3 granitoids } \\
\hline Morro do Afonso Syenite & $2111 \pm 10$ & & $2641 \pm 4$ & SHRIMP & Rios et al. (2007) \\
\hline Itareru Lamprophyre & $2113 \pm 5$ & & & SHRIMP & This work \\
\hline Itareru Diorite & $2106 \pm 2$ & & $2162 \pm 4$ & ID-TIMS & This work \\
\hline Serra do Pintado Syenite & $2098 \pm 2$ & & & ID-TIMS & Conceição et al. (2002) \\
\hline Euclides Shoshonite Syenogranite & $2097 \pm 8$ & & 3654 and $2344-2501$ & ID-TIMS/SHRIMP & Rios et al. (2008) \\
\hline Pedra Vermelha Biotite-Granite & $2080 \pm 8$ & & & ID-TIMS & This work \\
\hline Barroquinhas Biotite-Granite & $2073 \pm 1$ & & & ID-TIMS & This work \\
\hline Faz.Bananas Biotite-Monzogranite & $2072 \pm 1$ & & & ID-TIMS & This work \\
\hline \multicolumn{6}{|l|}{ Late Intrusions and Mineralization } \\
\hline Dykes cutting Ambrósio & $2080 \pm 2(\mathrm{Xe})$ & & & SHRIMP & Mello et al. (2006) \\
\hline Dykes cutting Ambrósio & $2079 \pm 47(\mathrm{Zr})$ & & & ID-TIMS & Gaal et al. (1987) \\
\hline Quartz-Feldspar Porphyry Dyke cutting Teofilandia & $2128 \pm 8$ & $2071 \pm 9$ & & SHRIMP & Mello et al. (2006) \\
\hline
\end{tabular}




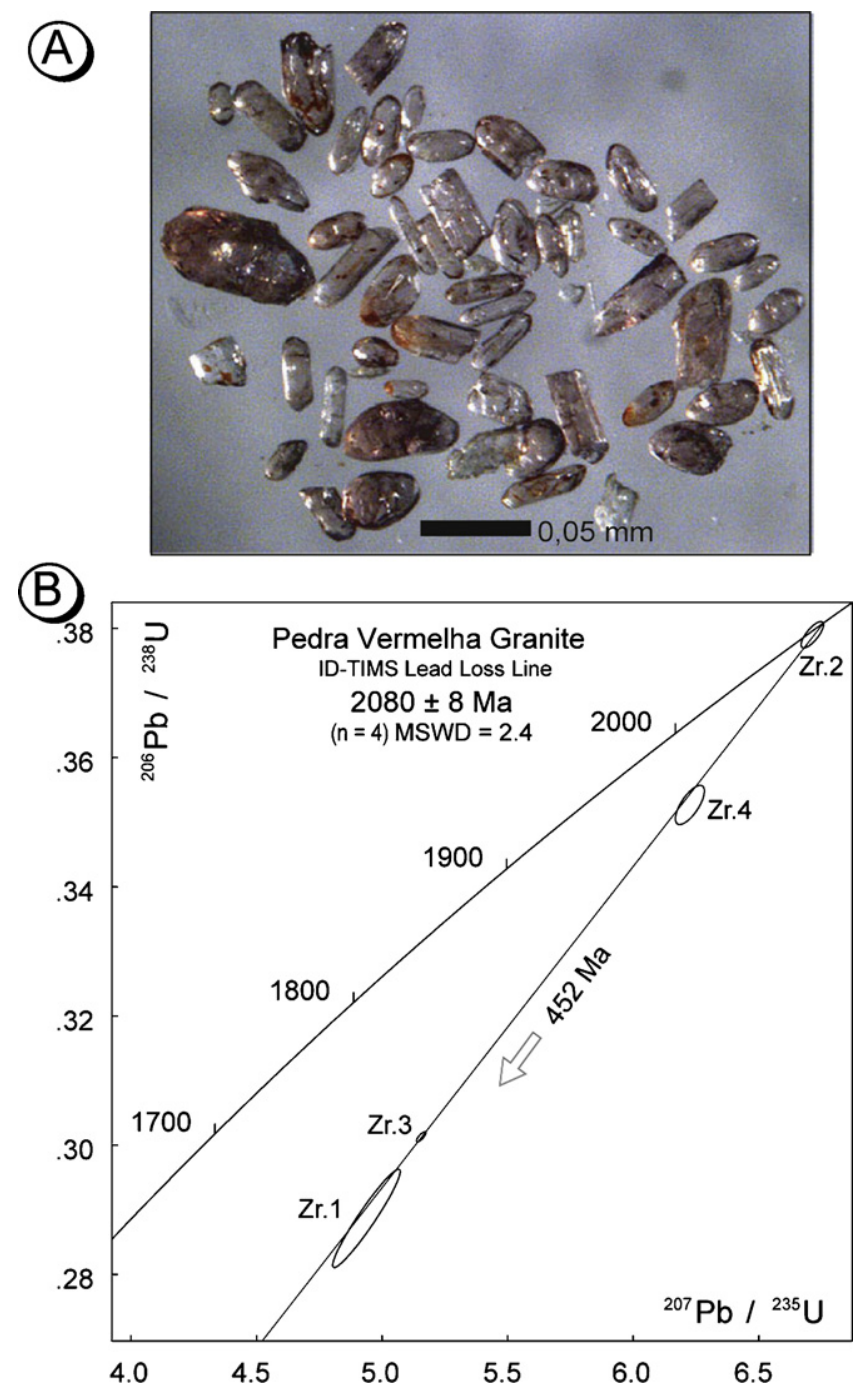

Fig. 13. Sample 1664-Pedra Vermelha Granite. (A) Zircon populations. (B) ID-TIMS Concordia diagram.

these alkaline magmas, beginning with syenite and shoshonite plutons at about $2110 \mathrm{Ma}$. A tectono-thermal metamorphic event is recorded by the emplacement of ellipsoidal syenite and shoshonite plutons, such as the Euclides (2097 $\pm 8 \mathrm{Ma}$, U-Pb, Rios et al., 2002, 2008), Cansanção (2105 $\pm 3 \mathrm{Ma}, \mathrm{Pb}-\mathrm{Pb})$, and the Itareru (2106 $\pm 2 \mathrm{Ma}, \mathrm{U}-\mathrm{Pb}$, this work) plutons, the lamprophyric dykes (intruding Itareru shoshonite, $2113 \pm 5 \mathrm{Ma}, \mathrm{U}-\mathrm{Pb}$, this work), and three syenitic stocks: Morro do Afonso $(2111 \pm 10 \mathrm{Ma}, \mathrm{U}-\mathrm{Pb}$, Rios et al., 2007), Serra do Pintado (2098 \pm 2 Ma, U-Pb, Conceição et al., 2002) and Agulhas-Bananas (2086 $\pm 2 \mathrm{Ma}, \mathrm{Pb}-\mathrm{Pb}$, Conceição et al., 2002). 2.07-2.08 Ga magmatic and metamorphic overgrowths are also found on zircon in older rocks.

The greatest surprise, and perhaps one of the most important results of this study, is the significant number of Mesoarchaean ages. The $\mathrm{Sm}-\mathrm{Nd}$ and $\mathrm{Rb}-\mathrm{Sr}$ systems from group 1 plutons also yield $T_{\mathrm{DM}}$ ages that are similar to the zircon ages reported here (Table 3, Fig. 2). Group 1 plutons in the Rio Itapicuru greenstone belt and Serrinha nucleus gneissic basement are N-S elongated and have foliated margins, whereas their cores are more isotropic (Matos and Davison, 1987). They are similar to TTG Archaean suites of Martin (1994), with strongly fractionated REE patterns, concave terminations and small Eu anomalies (Rios, 2002). Many

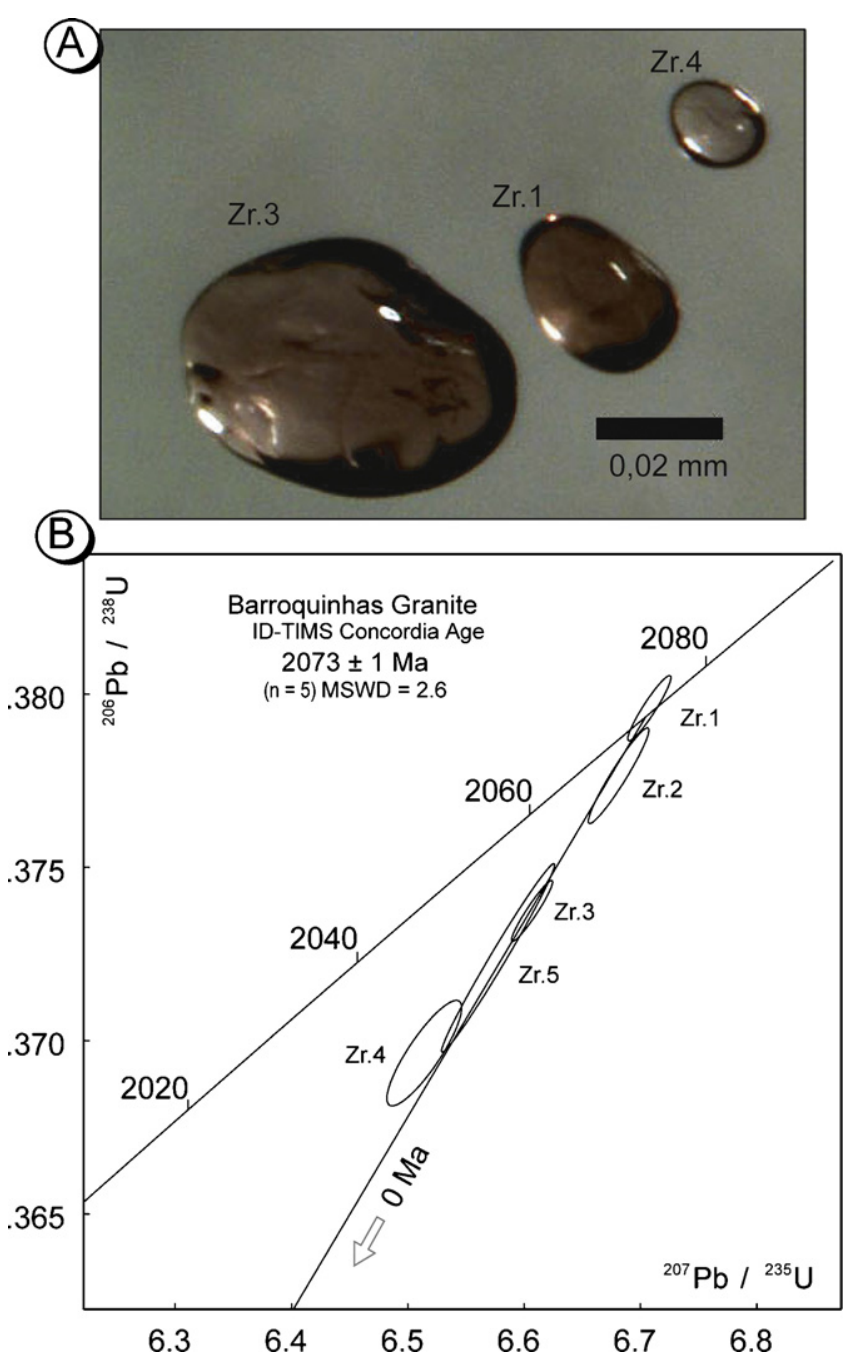

Fig. 14. Sample 1587-Barroquinhas Granite. (A) Zircon populations. (B) ID-TIMS Concordia diagram.

of the largest bodies in Serrinha nucleus can be considered as belonging to group 1, including some of the pre- and syntectonic granites of Matos and Conceição (1993). Many of these plutons clearly cut the mafic basal unit of the Itapicuru greenstone belt (Argolo, 1993; Alves da Silva et al., 1993; Chauvet et al., 1997a,b; Lacerda, 2004; Mello et al., 2006), including the Ambrósio, the Pedra Alta and the Poço Grande plutons (Fig. 2). Volcanism and sedimentation within the Rio Itapicuru greenstone belt is Paleoproterozoic in age, based on previous dating (Silva, 1987, Silva et al., 1996) and new SHRIMP data (in preparation). Therefore, the Mesoarchean complexes must have been emplaced within the greenstone belt as a result of remobilization during the Transamazonian orogeny. They may represent diapirs or cores of crustal-scale folds.

The São Francisco craton contains evidence of at least three major Archaean tectonic-thermal and magmatic events (3380-2900 Ma, 2860-2800 Ma, 2780-2600 Ma; Machado and Carneiro, 1992; Carneiro, 1992; Wilson et al., 1995; Martin et al., 1997), of which the two younger events involved partial reworking of older sialic crust. These authors also found evidence of inherited components up to $3473 \mathrm{Ma}$ and tentatively proposed the existence of a ca. $3.66 \mathrm{Ga}$ old proto-continental crust (Martin et al., 1991, 1997). Xenocrysts of this age have now been found in the groups 2 and 3 plutons (Quijingue and Euclides plutons, Rios et al., 2008). 

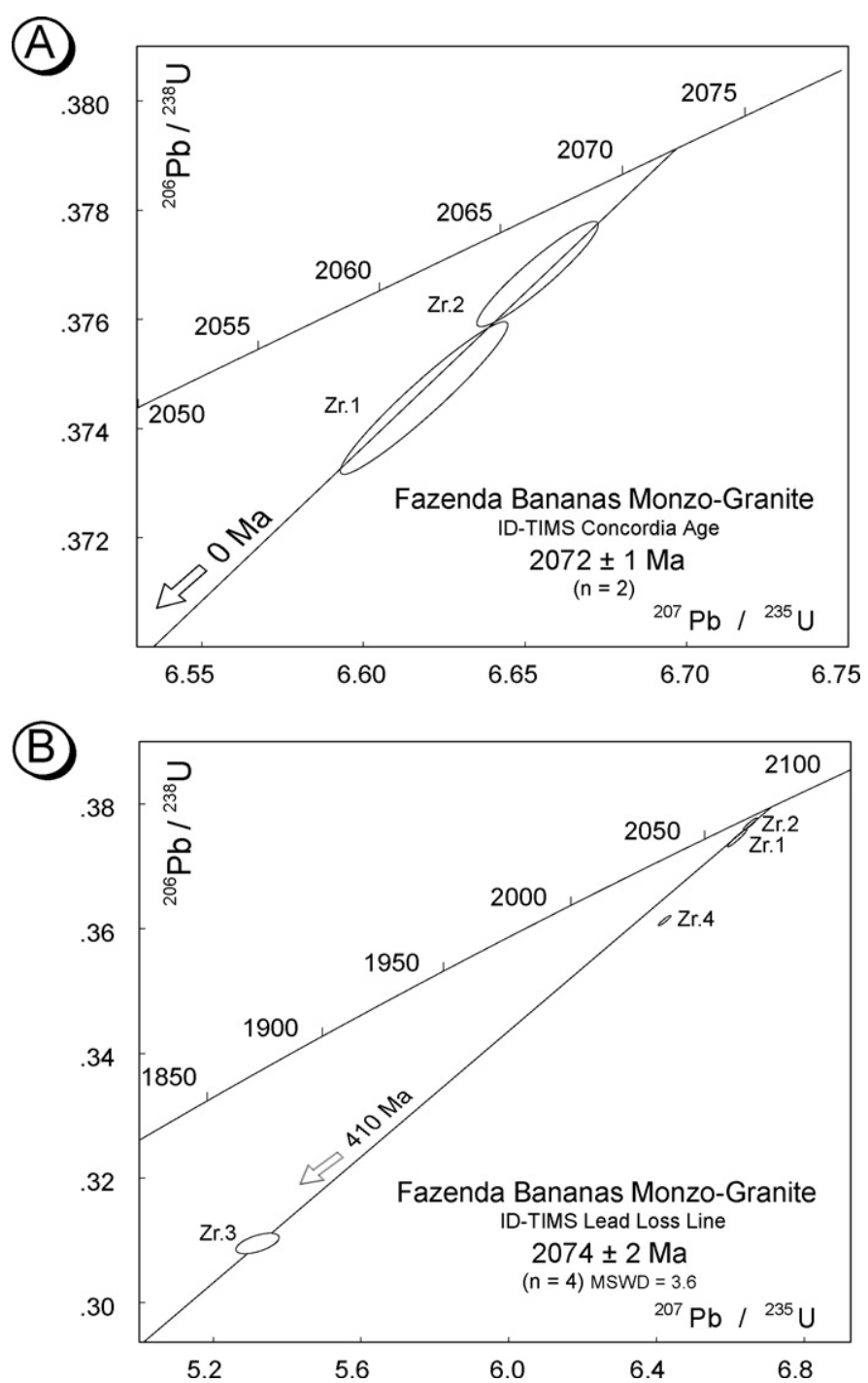

Fig. 15. Sample 1436-Fazenda Bananas Monzo-Granite. ID-TIMS Concordia diagram. (A) Regression to origin. (B) Lead loss line.

Large N-S elongated plutons like Ambrósio are also described in other regions of the São Francisco craton (e.g. Sete Voltas Massif, Martin et al., 1991, 1997). Based on the present data, we suggest that most large N-S elongated plutons are Archaean in age. In this study, there is little evidence for geological events over the period 2.9-2.2 Ga, with the exception of the pattern of zircon SHRIMP ages from the Euclides pluton, in which ages range from 2087 to $2501 \mathrm{Ma}$ (Rios et al., 2008).

The earliest Palaeoproterozoic granitic units of group 2 have normal calc-alkaline to subordinate TTG trends and metaluminous to peraluminous character (Rios, 2002). Chemically, they are very similar to group 1 granitoids, although they are less deformed, less elongated, and show more juvenile isotopic compositions (Table 4). They are located in the central portion of the Serrinha nucleus, most along the edge of the Itapicuru greenstone belt where they intrude the Archaean gneiss-migmatite and the volcano-sedimentary rocks. Like the group 1 granitoid bodies, most of them show a gneissic border that grades to an isotropic core (Rios, 2002), but they appear to be regionally less deformed than the Archaean plutons.

Xenoliths of Itapicuru rocks as well as gneiss-migmatite and mafic micro-granular enclaves are frequent. Meso- to Eoarchaean zircons, interpreted as basement xenocrysts, are found in group 2 plutons (Rios, 2002; Rios et al., 2002, 2008). 2.07-2.08 Ga overgrowths are also identified. Most of the plutons are peraluminous, except Nordestina, which shows metaluminous character. HREE values are lower than those of post-Archaean suites of Martin et al. (1997), suggesting they were generated in the garnet stability zone (Rios, 2002; Cruz Filho et al., 2005).

Younger alkaline magmatism (group 3) makes up a distinct but volumetrically minor assemblage of rock types that is most prominently developed in the southwestern area of the nucleus. Plutons occur as small, semi-circular $\left(<30 \mathrm{~km}^{2}\right)$ bodies and dykes, comprising three geochemically distinctive sub-groups: (i) shoshonite (Euclides, Araras, Cansanção e Itareru), (ii) potassic-ultrapotassic (Morro do Afonso, Agulhas-Bananas, and Serra do Pintado), and (iii) K-peraluminous granites (Morro do Lopes type).

Serrinha nucleus shoshonite and syenite bodies have a slightly ellipsoidal, N-S elongated form, and some of them also show a slightly foliated border, in which basement xenoliths have been identified. These foliations are discordant to the regional pattern, have magmatic character, and there are no other deformational textures (Nascimento, 1996; Oliveira, 2001; Rios et al., 2007; Nascimento et al., 2004), indicating that they intruded near the end of the Transamazonian orogeny. Syenite bodies are aligned closely with the $39^{\circ}$ meridian, suggesting that their emplacement was controlled by a deep regional fault structure.

Both syenite and shoshonite are characterized by high LILE and HFSE contents (Conceição et al., 1995, 2002; Rios, 2002; Plá Cid et al., 2006; Rios et al., 2007). According to Foley et al. (1987), Foley and Peccerillo (1992) and Ringwood (1990), rocks with the characteristics of Serrinha nucleus syenites are probably related to a deep mantle source, and influenced by subduction-enriched mantle and contamination with continental crust (Thompson and Fowler, 1986).

The available data allow us to say that syenitic and shoshonitic magmas are coeval and related to lamprophyric magmas and to constrain the time of their emplacement at 2097-2113 Ma, as well as to confirm the presence of crustal participation in their genesis as shown by zircon inheritance and Neoarchaean $T_{\mathrm{DM}}$ ages (Table 4 ).

\subsection{Inferred tectonic and regional implications}

The present data, along with other isotopic and geochronological studies, indicate that the Serrinha nucleus shows a long history of reworking in the Mesoarchaean. Major crust forming events are dominantly in the range 2989-3162 Ma, since rocks of these ages appear to be regionally extensive. Mesoarchaean crust may have been formed on an older $3.6 \mathrm{Ga}$ basement as shown from xenocrysts.

The new ages of $3200 \pm 12$ Ma for a Uauá complex gneissmigmatite near Quijingue and $3162 \pm 26 \mathrm{Ma}$ from the Ambrósio tonalite extend the known rock record in the Serrinha nucleus to $3.20 \mathrm{Ga}$, closer to the $3.34 \mathrm{Ga}$ mantle extraction ages indicated from Nd isotopes (Oliveira et al., 1999, 2000, 2002), whereas a 2.16-Ga TTG body (Quijingue) contains inheritance up to $3.61 \mathrm{Ga}$ in age (Rios et al., 2002). Thus, Archean phases in Serrinha nucleus appear to have developed by progressive reworking of pre-existing crust. In terms of its age distribution, this older crust somewhat resembles parts of the Kaapvaal and Pilbara cratons, where 3.1-3.3 Ga rocks are abundant (Kamo and Davis, 1994; Van Kranendonk et al., 2007). There is a notable lack of evidence for Neoarchean activity, in contrast to many other Archean cratons.

The Transamazonian orogeny is one of the most important crustal formation events in South America. Santos et al. (2003) present the precise time span of the orogenic cycle, and identify four main orogenies, although other authors have proposed that at least 
12 orogenic pulses may have occurred (Hartmann et al., 2004). In the Serrinha nucleus, we identify two main Transamazonian pulses at 2163-2128 Ma and 2110-2070 Ma.

Tectonic interpretations in ancient highly deformed terranes like Serrinha nucleus are necessarily limited. Nevertheless, available geochemistry, isotope and age data are suggestive of that the Paleoproterozoic rocks of Serrinha nucleus developed as an arc on the (present-day) southern edge of a Mesoarchaean continental margin. Itapicuru and Capim greenstone belts (Fig. 2) likely represent the remains of this arc. These rocks were previously interpreted on the basis of geochemistry to have formed in backarc basins (Kishida and Riccio, 1980; Silva, 1992, 1996; Winge, 1984; Figueiredo, 1989). Coeval group 2 granitoids, most with ages near $2160 \mathrm{Ma}$, also show geochemical arc affinities. They contain Mesoarchaean xenocrysts and cores and show Archean Nd mantle extraction ages. They were deformed later during the Transamazonian orogeny.

The 2.07-2.11 Ga group 3 granitoid rocks mainly comprise late to post-tectonic plutons that must have been associated with a considerable input of heat as shown by similar age zircon rims in older rocks and by many of the $\mathrm{Rb}-\mathrm{Sr}, \mathrm{K}-\mathrm{Ar}, \mathrm{Ar}-\mathrm{Ar}$ and $\mathrm{Pb}-\mathrm{Pb}$ isotopic data measured on older granitoid and felsic volcanic rocks (see Table 1). This pattern of arc-associated magmatism followed by regional deformation and intense late- to post-tectonic plutonism is characteristic of Archaean collision tectonics but in some areas apparently lasted into the Palaeoproterozoic. A distinct suite of $\mathrm{Mg}$-rich rocks enriched in both compatible and incompatible elements (the sanukitoid suite) is suggested to form from melting of mantle previously enriched by subduction processes (Shirey and Hansen, 1984; Stern and Hansen, 1991). Similar rocks have been recognized in the Serrinha nucleus (Itareru pluton, $\sim 2.1 \mathrm{Ga}$, Rios et al., 2000). Enrichment of the source mantle most likely occurred during earlier phases of Palaeoproterozoic or Archaean subduction. The thermal pulse necessary to melt this mantle in a post-subduction environment may have been from a sudden influx of asthenospheric mantle following collision and slab break off (Davies and Von Blanckenburg, 1995). A similar model has been proposed for late plutonism in the Neoarchaean Superior province (Beakhouse and Davis, 2005). Thus, different aged Palaeoproterozoic rocks that make up the Transamazonian 'cycle' likely did not form during multiple orogenies but during different phases of the same ocean closure event.

Mello et al. (2006) suggest that the peak of the tectonometamorphic-magmatic activity related to the formation of the Itapicuru basin must have occurred during a collisional period between $2130 \mathrm{Ma}$ and $2080 \mathrm{Ma}$. The thermal event related to the emplacement of Morro do Lopes type granites (2.07-2.08 Ga) agrees with ${ }^{40} \mathrm{Ar} /{ }^{39} \mathrm{Ar}$ biotite and muscovite ages measured for gold mineralization, which are bracketed between ca. $2083 \mathrm{Ma}$ and $2031 \mathrm{Ma}$ (Vasconcelos and Becker, 1992; Mello et al., 2006), suggesting that this late plutonic activity could be responsible for the hydrothermal activity that transported and concentrated gold. Fluids may have been of magmatic origin and/or derived from dehydration of buried supracrustal units. Other authors have suggested a similar model and that the gold concentration process post-dates Transamazonian ductile deformation (horizontal thrusting and vertical movement; Alves da Silva et al., 1998).

Group 2 and group 3 plutons are coeval with similar units identified in West Africa (Hirdes et al., 1992, 1996; Doumbia et al., 1998) and Guiana (Norcross et al., 2000) although, in contrast to Serrinha nucleus, the West Africa rocks seem to be almost entirely juvenile (Davis et al., 1994). Thus, the Transamazonian-Eburnean Orogeny was probably very extensive and involved both oceanic and continental arc construction. The African equivalent of Serrinha nucleus may be found elsewhere, perhaps in the Congo craton.

\section{Conclusions}

The major events that affected the region are as follows:

Ca. 3.6 Ga to 3.2 Ga: Formation of Eo to Mesoarchaean crust. 3.2 to $2.9 \mathrm{Ga}$ : Intrusion of group 1 granitoids, which are associated with, or postdated, greenstone belt formation. These granitoids have typical features of Archaean TTGs, implying varying degrees of partial melting of underplated crust with an Archaean metamorphic event at $\sim 3070 \mathrm{Ma}$.

2.16 to $2.13 \mathrm{Ga}$ : Intrusion of group 2 granitoids in a continental arc environment floored by Mesoarchaean crust.

2.11 to $2.07 \mathrm{Ga}$ : Intrusion of group 3 granitoids, following ocean closure and slab break off. The geochronology, geochemical and spatial associations and lithostratigraphy indicate two stages of alkaline magmatism: a first stage (2114-2097 Ma), with intrusion of shoshonite, syenite and ultrapotassic lamprophyric rocks; and a second stage (ca. 2080-2070 Ma), with intrusion of small semi-circular peraluminous isotropic K-granites and dykes. These were the probable heat source driving Palaeoproterozoic metamorphism at $\sim 2070-2080$ Ma.

If these interpretations are correct, evidence for at least two major phases of regional deformation and metamorphism (Archaean and Transamazonian) should be present in at least some of the group 1 plutons, whereas only the Transamazonian events should have affected group 2 plutons.

\section{Acknowledgements}

We would like to express our appreciation to the members of the Jack Satterly Laboratory (University of Toronto) and Ion Microprobe at the Geological Survey of Canada for their instruction, assistance and patience during this research. Detailed comments on the manuscript by anonymous reviewers are also greatly appreciated. Financial assistance from the Conselho Nacional de Desenvolvimento Científico e Tecnológico (CNPq; 471170/20062, 304081/2006-0), Fundação Coordenação de Aperfeiçoamento do Pessoal do Ensino Superior (CAPES; process BEX 1332-98/8), Companhia Baiana de Pesquisa Mineral (CBPM), and Fundação de Amparo a Pesquisa do Estado da Bahia (FAPESB, INT004/2006) is gratefully acknowledged. The paper forms part of Ph.D. and Postdoctoral studies carried out by D.C. Rios at Federal University of Bahia (Brazil). This is GPA contribution 244.

\section{References}

Alves da Silva, F.C., Chauvet, A., Faure, M., 1993. Early Proterozoic (Transamazonian) Orogeny and sin-tectonic granite emplacement in the RIGB, Bahia, Brazil. Académie des Sciences, Paris, 316(2), 1139-1146.

Alves da Silva, F.C., Chauvet, A., Faure, M., 1998. General features of the gold deposits in the Rio Itapicuru Greenstone belt (RIGB, NE Brazil), discussion of the origin, timing and tectonic model. Rev. Bras. Geociencias. 28 (3), 377-390.

Argolo, J.L., 1993. Evolution Archeénne et Proterozóï que Inférieur de la partie NordOrientale du Craton de San Francisco. Ph.D. Thesis, Univ. Paul Sabatier-Toulouse III, France, $139 \mathrm{p}$

Barbosa, J., Sabaté, P., 2004. Archaean and Palaeoproterozoic crust of the São Francisco Craton, Bahia, Brazil: geodynamic features. Precambrian Res. 133, 1-27.

Barbosa, J.S.F., Dominguez, J.M.L. (Coords.), 1996. Texto Explicativo para o Mapa Geológico do Estado da Bahia-Esc. 1:1.000.000. Secretaria da Indústria, Comércio e Mineração do Estado da Bahia, SGM/PPPG/FAPEX/CPGG. Salvador-BA, 295 p.

Barrueto, H.R., 1997. Intrusões sub-vulcânicas alcalinas e lamprófiros nas mineralizações auríferas do GBRI, Bahia: petrografia, geoquímica e inclusões fluidas. Dissert. Mestrado. Pós-Graduação em Geociências. Unicamp-SP. 160 p.

Bastos Leal, L.R., 1992. Geocronologia Rb-Sr e K/Ar, evolução isotópica e implicações tectônicas dos enxames de diques máficos de Uauá e Vale do Rio Curaçá, Bahia. Dissertation. Mestrado, USP, 120 p.

Bastos Leal, L.R., Teixeira, W., Piccirillo, E.M., Leal, A.B.M., Girardi, V.A.V., 1994. Geocronologia Rb/Sr e K/Ar do enxame de diques maficos de Uauá, Bahia (Brasil). Geoch. Brasilienses 8, 99-114. 
Batista, J.J., Nesbitt, R.W., Pires, P.F.R., 1998. Presença do embasamento arqueano no Greenstone Belt do Rio Itapicuru (Bahia)-resultados geocronológicos por ICPMS-LA. XL Congresso Brasileiro de Geologia. Belo Horizonte, MG. SBG. Anais, p. 67.

Beakhouse, G.P., Davis, D.W., 2005. Evolution and tectonic significance of intermediate to felsic plutonism associated with the Hemlo greenstone belt, Superior Province, Canada. Precambrian Res. 137, 61-92.

Bellieni, G., Piccirillo, E.M., Petrini, R., Girardi, V.A.V., Menezes Leal, A.B., Teixeira, W. Bastos Leal, R.R., De Amin, A., Comin Chiaromonti, P., Tanner de Oliveira, M.A.F., 1995. Petrological and $\mathrm{Sr}-\mathrm{Nd}$ evidence bearing on early proterozoic magmatic events of the sub-continental mantle: São Francisco Craton (Uauá, NE Brazil). Contrib. Mineral. Petrol. 122 (3), 252-261.

Beurlen, H., 1970. Estudos petrograficos no embasamento cristalino - Fazenda Rodeador - Uauá - Bahia, Serie Especial numero 12. Divisao de Geologia/SUDENE, Recife.

Brito Neves, B.B., Cordani, U.G., Torquato, J.R.F., 1980. Evolução Geocronológica do Pré-Cambriano do Estado da Bahia, in: Inda, H.A.V., Duarte, F.B. (Eds.), Geologia e Recursos Minerais do Estado da Bahia. Sec. Minas do Estado da Bahia, Textos Básicos. SME/CPM, 3: 1-101.

Carneiro, M.A., 1992. O complexo metamorfico Bonfim Setentrional: evolucao geologica de um segmento arqueano de crosta continental. Ph.D. Thesis, Univ. São Paulo, São Paulo, 232 pp.

Chauvet, A., Alves da Silva, F.C., Faure, M., Guerrot, C., 1997a. Structural evolution of the Paleoproterozoic Rio Itapicuru granite-greenstone belt (Bahia, Brazil): the role of synkinematic plutons in the regional tectonics. Precambrian Res. 84, $139-162$.

Chauvet, A., Guerrot, C., Alves da Silva, E.C., Faure, M., 1997b. Geochronologie ${ }^{207} \mathrm{~Pb} /{ }^{206} \mathrm{~Pb}$ and ${ }^{40} \mathrm{Ar} /{ }^{39} \mathrm{Ar}$ des granites paleoproterozoiques de la ceinture de roches vertes du Rio Itapicuru (Bahia, Brasil). C. R. Acad. Sci. Paris II 324, 293-300.

Conceição, H., Rios, D.C., Rosa, M.L.S., 1995. Petrologia da Associação SienitoLamprófiro: Caso da Intrusão de Morro do Afonso (Greenstone Belt do Rio Itapicuru, Bahia). Geoch. Brasilienses 9 (1), 91-109.

Conceição, H., Rios, D.C., Rosa, M.L.S., Davis, D.W., Dickin, A.P., McReath, I., Marinho, M.M., Macambira, M.J.B., 2002. Zircon geochronology and petrology of alkalinepotassic syenites, Southwestern Serrinha Nucleus, East São Francisco Craton, Brazil. Int. Geol. Rev. 44 (2), 117-136.

Cordani, U.G., Isotta, C.A.L., Teixeira, W., 1969. Reconhecimento geocronológico do embasamento da região oriental do Estado da Bahia. In: Proceedings of 23 Congresso Brasileiro de Geologia, Anais, Salvador, pp. 159-165.

Cordani, U.G., Milani, E.J., Thomaz Filho, A., Campos, D.A. (Eds.), 2000. Tectonic Evolution of South America. 31st International Geological Congress. Rio de Janeiro, RJ, 856 p.

Cordani, U.G., Sato, K., Nutman, A., 1999. Single zircon SHRIMP determination from Archaean tonalitic rocks near Uauá, Bahia, Brazil. In: Proceedings of the Second South American Symposium on Isotope Geology, Cordoba-Argentina, pp. 27-30.

Cruz Filho, B.E., Conceição, H., Rosa, M.L.S., Rios, D.C., Macambira, M.J.B., Marinho, M.M., 2005. Geocronologia e assinatura isotopica (Rb-Sr e Sm-Nd) do Batolito Trondhjemitico Nordestina, Nucleo Serrinha, Nordeste do Estado da Bahia. Rev. Bras. Geociencias 35 (4-suplemento), 1-8.

Davies, J.H., Von Blanckenburg, F., 1995. Slab breakoff: a model of lithosphere detachment and its test in the magmatism and deformation of collisional orogens. Earth Planet. Sci. Lett. 129, 85-102.

Davis, D.W., 1982. Optimum linear regression and error estimation applied to U-Pb data. Can. J. Earth Sci. 19, 2141-2149.

Davis, D.W., Gray, J., Cumming, G.L., Baadsgaard, H., 1977. Determination of the ${ }^{87} \mathrm{Rb}$ decay constant. Geochim. et Cosmochim. Acta 41, 1745-1749.

Davis, D.W., Hirdes, W., Schaltegger, U., Nunoo, E.A., 1994. U-Pb age constraints on deposition and provenance of Birimian and gold-bearing Tarkwaian sediments in Ghana, West Africa. Precambrian Res. 67, 89-107.

Davison, I., Teixeira, J.B.G., Silva, M.G., 1988. The Itapicuru Greenstone Belt, Bahia, Brazil: structure and stratigraphical outline. Precambrian Res. 42 (1-2), 1-17.

De Paolo, D.J., Schubert, G., 1991. The continental age distribution: methods of determining mantle separation ages from $\mathrm{Sm}-\mathrm{Nd}$ isotopic data and application to the southwestern United States. J. Geophys. Res. 96, 2071-2088.

Doumbia, S., Pouclet, A., Kouamelan, A., Peaucat, J.J., Vidal, M., Delor, C., 1998. Petrogenesis of juvenile-type Birimian (Palaeoproterozoic) granitoids in Central Côte-d'Ivoire, West Africa: geochemistry and geochronology. Precambrian Res. $87,33-63$.

Faure, G., 1986. Principles of Isotopic Geology. Wiley and Sons, New York, 589 p.

Figueiredo, M.C.H., 1989. Geochemical evolution of eastern Bahia, Brazil: a probably early proterozoic subduction-related magmatic arc. J. South Am. Earth Sci. 2 (2), 131-145.

Foley, S.F., Peccerillo, A., 1992. Potassic and ultrapotassic magmas and their origin. Lithos 28, 181-185.

Foley, S.F., Venturelli, G., Green, D.H., Toscani, L., 1987. The ultrapotassic rocks: characteristics, classification and constrains for petrogenetic models. Earth Sci. Rev. 24, 81-134.

Gaal, G., Teixeira, J.B.G., Silva, M.G., Del Rey, J.M.H., 1987. New U-Pb data from granitoids, reflecting early proterozoic evolution in northeast Bahia-Brazil. International Symposium on Granites and Associated Mineralizations. Salvador, Bahia, Brazil (unpublished).

Hartmann, L.A., Endo, I., Suita, M.T.F., Santos, J.O.S., Frantz, J.C., Carneiro, M.A., McNaughton, N.J., Barley, M.E., 2006. Provenance and age delimitation of Quadrilátero Ferrífero sandstones based on zircon U-Pb isotopes. J. South Am. Earth Sci. 20, 273-285.
Hartmann, L.A., Philipp, R.P., Liu, D., Wan, Y., Wang, Y., Santos, J.O.S., Vasconcellos, M.A.Z., 2004. Palaeoproterozoic magmatic provenance of detrital zircon crystals, porongos complex quartzites, southern Brazilian shield. Int. Geol. Rev. 46, 127-157.

Hirdes, W., Davis, D.W., Eisehlohr, B.N., 1992. Reassessment of proterozoic granitoid ages in Ghana on the basis of $\mathrm{U} / \mathrm{Pb}$ zircon and monazite dating. Precambrian Res. 56, 89-96.

Hirdes, W., Davis, D.W., Ludtke, G., Konan, G., 1996. Two generations of Birimian (Palaeoproterozoic) volcanic belts in northeastern Côte d'Ivoire (West Africa): consequences for the "Birimian controversy". Precambrian Res. 80, 173191.

Inda, H.A.V., Souza, A.G., Silva Filho, A.A., Pires, A.B., Portela, A.C.P., Cavedon, A.D., Sanchez, B.A., Santos, E.Z., Pereira, F.S., Goncalves, J.C., Neto, L.F.B., Costa, M.R.A., Damiao, R.N., Nasamann, R., Oliveira, V., 1976. Projeto Rochas Básicas e Ultrabásicas de Euclides da Cunha. Relatório Final. Rio de Janeiro, PROSPEC, SME-BA. 12 volumes.

Jaffey, A.H., Flynn, K.F., Glendenin, L.E., Bentley, W.C., Essling, A.N., 1971. Precision measurement of half-lives and specific activities of ${ }^{235} \mathrm{U}$ and ${ }^{238} \mathrm{U}$. Phys. Rev. 4, 1889-1906.

Jardim de Sá, E.F., 1982. Nota sobre o estilo estrutural e relações gnaisses vs. supracrustais no Greenstone Belt de Serrinha, Ba. Ciências da Terra 2, 8-13.

Kamo, S., Davis, D.W., 1994. Reassessment of Archaean crustal development in the Barberton mountain land, South Africa, based on U-Pb dating. Tectonics 13, 167-192.

Kishida, A., Riccio, L., 1980. Chemostratigraphy of lava sequence from the Rio Itapicuru Greenstone Belt, Bahia, Brazil. Precambrian Res., 161-178.

Krogh, T.E., 1973. A low-contamination method for hydrothermal decomposition of zircon and extraction of $\mathrm{U}$ and $\mathrm{Pb}$ for isotopic age determinations. Geochim. Cosmochim. Acta 37 (3), 485-494.

Krogh, T.E., 1982. Improved accuracy of U-Pb zircon ages by the creation of more concordant systems using air abrasion technique. Geochim. Cosmochim. Acta $46,637-649$.

Lacerda, C.M.M., 2004. Evolucao estrutural e petrogenetica do Domo Granodioritico de Ambrósio. Unpub.: Ph.D. Thesis. Universidade Estadual de Campinas.

Ludwig, K.R., 2001. Squid1. 02: A User Manual. Berkeley Geochronological Center Special Publication no. 2, 19 p.

Lugmair, G.W., Marti, K., 1978. Lunar initial 143Nd/144Nd: Differential evolution of the lunar crust and mantle. Earth Planet. Sci. Lett. 39, 349-357.

Machado, N., Carneiro, M.A., 1992. A major Archaean tectonothermal event in the São Francisco shield, Brazil: U/Pb evidence from the Quadrilatero Ferrifero, Minas Gerais. Can. J. Earth Sci. 29 (11), 2341-2346.

Martin, H., 1994. The Archaean grey gneisses and the genesis of continental crust In: Condie, K.C. (Ed.), Developments in Precambrian Geology, vol. 11. Elsevier Amsterdam, pp. 205-225.

Martin, H., Sabate, P., Peaucat, J.-J., Cunha, J.C., 1991. Un segment de la crôute continentale d'âge archéean ancien (3.4 milliards d'années): le massif de Sete Voltas (Bahia, Brésil). Comptes Rendus de l'Acad. Sci. Paris 313, 31-538.

Martin, H., Sabate, P., Peaucat, J.-J., Cunha, J.C., 1997. Crustal evolution in early archaean of South America: example of the Sete Voltas Massif, Bahia state, Brazil. Precambrian Res. 82, 35-62.

Mascarenhas, J.F., Garcia, T.W., 1987. Mapa geocronológico do Estado da Bahia: Texto Explicativo. Superintendência de Geologia e Recursos Minerais, Sec. Minas e Energia, Salvador-BA, 186 p.

Mascarenhas, J.F., Pedreira, A.J., Misi, A., Motta, A.C., Sa, J.H.S., 1984. Província São Francisco, in: Almeida, F.F.M., Hasuy, Y. (Orgs.) (Eds.), O Pré-Cambriano do Brasil. São Paulo, Edgard Blusher, 4, 46-122.

Mascarenhas, J.F., Pedreira, A.J.C.L., Gil, C.A.A., Neves, J.P., Oliveira, J.E., Silva Filho, M.A., Marinho, M.M., 1979. Geologia da região centro-oriental da Bahia. In: Projeto Bahia - Bahia II - Sul da Bahia. Relatório integrado. CPRM/DNPM, Brasília $\mathrm{DF}, 128 \mathrm{p}$.

Mascarenhas, J.F., Sa, J.H.S., 1982. Geological and metallogenetic patterns in the archaean and early proterozoic of Bahia State, eastern Brazil. Rev. Bras. Geociencias 12 (3), 193-214.

Matos, F.M.V., 1988. Um estudo estrutural e petrográfico do Domo Granito-Gnáisse de Ambrósio no Greenstone Belt do Rio Itapicuru, Bahia, Brazil. Dissertação de Mestrado. CPG em Geologia, UFBA, 109 p.

Matos, F.M.V., Conceição, H., 1993. Granitogênese associada à parte oeste do Cráton Serrinha e o Greenstone Belt do Rio Itapicuru: Geologia e Tipologia. II Simp. Cráton do São Francisco. SBG/SGM/CNPq, Núcleo Bahia-Sergipe, Salvador-BA, Anais, pp. 60-62.

Matos, F.M.V., Davison, I., 1987. Basement or intrusion? The Ambrósio Dome, Rio Itapicuru Greenstone Belt, Bahia, Brazil. Rev. Bras. Geociencias 17 (4), 442-449.

Mello, E.F., Xavier, R.P., McNaughton, N.J., Hagemann, S.G., Fletcher, I., Snee, L., 2006. Age constraints on felsic intrusions, metamorphism and gold mineralization in the Paleoproterozoic Rio Itapicuru Greenstone Belt, NE Bahia State, Brazil. Miner. Dep. 40, 849-866.

Melo, R.C., Loureiro, H.S.C., Pereira, L.H.M., 1995. Programa Levantamentos Geológicos Básicos do Brasil. Serrinha-Folha SC.24-Y-D. Estado da Bahia, Brasil. Escala 1:250.000. CPRM/MME, Brasília-DF, 80 p.

Nascimento, H.S., 1996. Evolução petrogenética da intrusão ácido-básica de Cansanção. Mestrado, CPG em Geologia-UFBA, 205 p.

Nascimento, H.S., Bouchez, J.L., Nedelec, A., Sabate, P., 2004. Evidence of an early NS magmatic event in the Palaeoproterozoic Teofilandia granitoids (São Francisco Craton, Brazil): a combined microstructural and magnetic fabric study Precambrian Res. 134, 41-59. 
Norcross, C., Davis, D.W., Spooner, E.T.C., Rust, A., 2000. U-Pb and Pb-Pb age constraints on Palaeoproterozoic magmatism, deformation and gold mineralization in the Omai Area, Guyana shield. Precambrian Res. 102, 69-86.

Oliveira, E.P., Lafon, J.-M., Souza, Z.S., 1998. A Palaeoproterozoic age for the Rio Capim volcano-plutonic sequence, Bahia, Brazil: whole-rock $\mathrm{Pb}-\mathrm{Pb}, \mathrm{Pb}$-evaporation and U-Pb constraints. In: Anais XI Congresso Brasileiro de Geologia, SBG, Belo Horizonte-MG, p. 14.

Oliveira, E.P., Lafon, J.-M., Souza, Z.S., 1999. Archaean-Paleproterozoic transition in the Uauá Block, NE São Francisco Craton, Brazil: $\mathrm{U}-\mathrm{Pb}, \mathrm{Pb}-\mathrm{Pb}$ and Nd isotope constraints. In: Proceedings of First International Symposium on Tectonics of the Brazilian Geological Society, Lençois-BA. Abstract-volume, pp. 49-51.

Oliveira, E.P., Mello, E.F., Mcnaughton, N., 2002. Reconnaissance U-Pb geochronology of precambrian quartzites from the Caldeirão belt and their basement, NE São Francisco craton, Bahia, Brazil: implications for the early evolution of the Palaeoproterozoic Itabuna-Salvador Curaçá orogen. J. South Am. Earth Sci. 15 (3), 349-362

Oliveira, E.P., Souza, Z.S., Correa-Gomes, L.C., 2000. U-Pb dating of deformed mafic dyke and host gneiss: implications for understanding reworking processes on the western margin of the Archaean Uauá Block, NE São Francisco Craton, Brazil. Rev. Bras. Geociencias 30 (1), 149-152.

Oliveira, E.P., Windley, B.F., McNaughton, N.J., Pimentel, M., Fletcher, I.R., 2004 Contrasting copper and chromium metallogenic evolution of terranes in the Palaeoproterozoic Itabuna-Salvador-Curaçá orogen, São Francisco craton, Brazil: new zircon (SHRIMP) and Sm-Nd (model) ages and their significance for orogen-parallel escape tectonics. Precambrian Res. 128 (1-2), 143-165.

Oliveira, L.L., 2001. Geologia e Litogeoquímica do magmatismo shoshonítico no nordeste do Núcleo Serrinha, Bahia: Maciço de Araras. Dissertation. Mestrado. CPG em Geologia, Univ. Federal da Bahia, 163 p.

Paixão, M.A.P., Lafon, J.M., Oliveira, E.P., 1995. Geocronologia $\mathrm{Pb}-\mathrm{Pb}$ do Complexo Anortositico-Leucogabróico de Lagoa da Vaca e rochas associadas, Complexo Uauá, Bahia. Congresso Brasileiro de Geoquimica, 5, Congresso de Geoquimica dos Paises de Lingua Portuguesa, 3, Niteroi, Anais em CD.

Paixão, M.A.P., Oliveira, E.P., 1998. The Lagoa da Vaca complex: an archaean layered anorthosite body on the western edge of the Uauá Block, Bahia, Brazil. Rev. Bras. Geociencias 28, 201-208.

Pereira, L.H.M., 1992. (Org.) Programa de Levantamentos Geológicos Básicos do Brasil (PLGB). Folha Serrinha (SC 24-Y-D-VI), Escala 1/100.000, Estado da Bahia. DNPM/CPRM, Brasília-DF, 180 p.

Pimentel, M.M., Silva, M.G., 2003. Sm-Nd Age of the Fazenda Brasileiro Gabbro, Bahia, Brazil: example of robust behavior of the Sm-Nd isotopic system under extreme hydrothermal alteration. Ann. Braz. Acad. Sci. 75 (3), 383-392.

Plá Cid, J., Rios, D.C., Conceição, H., 2006. Mafic minerals and geochemical aspects of mica-amphibole-bearing lamprophyres associated to the Palaeoproterozoic Morro do Afonso syenitic intrusion, Bahia, northeastern Brazil. J. South Am. Earth Sci. 22, 98-115.

Pupin, J.P., 1980. Zircon and granite petrology. Contrib. Mineral. Petrol. 73, 207-220.

Ringwood, A.E., 1990. Slab-mantle interactions: petrogenesis of intraplace magmas and structure of the upper mantle. Chem. Geol. 82, 187-207.

Rios, D.C., 2002. Granitogênese no Núcleo Serrinha, Bahia, Brasil: Geocronologia e Litogeoquímica. Unpub.: Ph.D. Thesis. Federal University of Bahia, 239 p.

Rios, D.C., Conceição, H., Davis, D.W., PlaCid, J., Rosa, M.L.S., Macambira, M.J.B., McReath, I., Marinho, M.M., Davis, W.J., 2007. Paleoproterozoic potassicultrapotassic magmatism: Morro do Afonso Syenite Pluton, Bahia, Brazil. Precambrian Res. 154, 1-30.

Rios, D.C., Conceição, H., Davis, D.W., Rosa, M.L.S., Macambira, M.J.B., Dickin, A.P., 2003. A new proposal for the subdivision of granitic rocks at Serrinha Nucleus, Bahia, Brazil, based on $\mathrm{U}-\mathrm{Pb}$ and $\mathrm{Pb}-\mathrm{Pb}$ geochronological and litogeochemical data. IV South American Symposium on Isotope Geology, Brazil. Short Papers, 1 264-267.

Rios, D.C., Conceição, H., Macambira, M.J.B., Burgos, C.M.G., Peixoto, A.A., Cruz Filho, B.E., Oliveira, L.L., Lisboa, 1998. Granitogênese da parte meridional-oriental do Núcleo Serrinha: idade, petrografia e geoquímica. In: Conceição, H., Cruz, M.J.M., Sá, H.J.S., Sabaté, P. (Eds.), Contribuição ao estudo dos Granitos e Rochas Correlatas, vol. 5. Pub. Esp. SBG, Núcleo Bahia-Sergipe, pp. 91-113.

Rios, D.C., Davis, D.W., Conceição, H., Macambira, M.J.B., Peixoto, A.A., Cruz Filho, B.E., Oliveira, L.L., 2000. Ages of granites of the Serrinha Nucleus, Bahia (Brazil): an overview. Rev. Bras. Geociencias 30, 74-77.
Rios, D.C., Davis, D.W., Conceição, H., Rosa, M.L., Dickin, A.P., 2004. Archaean granites at Serrinha nucleus, Bahia, Brazil. Geochim. Cosmochim. Acta 68 (11), A685.

Rios, D.C., Davis, D.W., Conceição, H., Rosa, M.L., Marinho, M.M., 2002. The oldest zircons from South America Continent. Geochim. Cosmochim. Acta 66 (A345), 640.

Rios, D.C., Davis, D.W., Conceição, H., Rosa, M.L.S., Davis, W.J., Dickin, A.P., Marinho, M.M., Stern, R., 2008. 3.65-2.10 Ga history of crust formation from zircon geochronology and isotope geochemistry of the Quijingue and Euclides plutons, Serrinha nucleus, Brazil. Precambrian Res. 167 (1-2), 53-70.

Sabate, P., Caen-Vachette, M., Marinho, M.M., Soares, C.P., 1990. Dados isotópicos $\mathrm{Rb}-\mathrm{Sr}$ e Sm-Nd da intrusão monzonítica a 2,0-Ga de Cansanção (Bahia-Brasil). XXXVI Congresso Brasileiro de Geologia. SBG. Bulletin de Résumés. Natal - RN, $4,163$.

Santos, J.O.S, Hartmann, LA. Bossi, J., Campal, N., Schipilov, A, Piñeyro, D. McNaughton, N.J., 2003. Duration of the trans-amazonian cycle and its correlation within South America based on U-Pb SHRIMP geochronology of the La Plata Craton, Uruguay. Int. Geol. Rev. 45, 27-48.

Shirey, S.B., Hansen, G.N., 1984. Mantle-derived Archaean monzodiorites and trachyandesites. Nature 310, 222-224

Silva, M.G., 1987. Geochimie, Petrologie und Geotektonisch Entwicklung eines Proterozoischen Grünsteignerfels Rio Itapicuru, Bahia Brasilien. Th (unpublished), Univ. Freiburg. RFA.

Silva, M.G., 1992. O Greenstone Belt do Rio Itapicuru: uma bacia do tipo back-arc fóssil. Rev. Bras. Geociencias 22, 157-166.

Silva, M.G., 1996. Síntese e Interpretação dos Dados Geocronológicos dos Terrenos Granito-Greenstone do Rio Itapicuru (Ba). XXXIX Cong. Bras. Geologia. Salvador, Bahia. Anais, 6, 544-547.

Silva, M.G., Coelho, C.E.S., Teixeira, J.B.G., Alves da Silva, F.C., Silva, R.A., Souza, J.A.B., 2001. The Rio Itapicuru greenstone belt, Bahia, Brazil: geologic evolution and review of gold mineralization. Miner. Dep. 36, 345-357.

Stern, R.A., 1997. The GSC sensitive high ion microprobe (SHRIMP): analytical techniques of zircon $\mathrm{U}-\mathrm{Th}-\mathrm{Pb}$ age determinations and performance evaluation. In Radiogenic and isotopes studies. Report 10, Geol. Survey of Canada. Current Research 1997-F, 1-31.

Stern, R.A., Hansen, G.N., 1991. Archaean high-Mg granodiorite: a derivative of light rare earth element-enriched monzodiorite of mantle origin. J. Petrol. 32, 201-238.

Teixeira, W., 1993. Avaliacao do acervo de dados geocronologicos e isotopicos do Craton do São Francisco-Implicacoes tectonicas. In: Craton do São Francisco: trabalhos apresentados na reuniao preparatoria do II Simposio do Craton do São Francisco, Salvador, Brasil, in: Dominguez, J.M.L., Misi, A. (Eds.), pp. 1133.

Thompson, R.N., Fowler, M.B., 1986. Subduction-related Shoshonitic and Ultrapotassic Magmatism: a study of Siluro-Ordovician Syenites from Scottish Caledonites. Contrib. Mineral. Petrol. 14, 507-522.

Van Kranendonk, M.J., Smithies, R.H., Hickman, A.H., Champion, D.C., 2007. Secular tectonic evolution of Archaean continental crust: interplay between horizontal and vertical processes in the formation of the Pilbara Craton, Australia. Terra Nova $19,1-38$

Vasconcelos, P., Becker, T., 1992. A idade da mineralização aurífera no depósito da Fazenda Brasileiro, Bahia, Brasil. Workshop em Metalogênese: Pesquisas atuais e novas tendências. UNICAMP, Bol. Res., 29.

Wilson, J.F.,Nesbitt, R.W., Fanning, C.M., 1995. Zircon geochronology of Archaean felsic sequences in the Zimbabwe craton: a revision of greenstone stratigraphy and amodel for crustal growth. In: Coward,M.P., Ries, A.C. (Eds.), Early Precambrian Processes. Geological Society of London. Special Publication 95, 109-126.

Wilson, M., 1989. Igneous Petrogenesis. Chapman \& Hall, London, 466 pp.

Winge, M., 1984. A seqüência vulcano-sedimentar do Grupo Capim, Bahia. In: Sa, P.V., Duarte, F.B. (Eds.), Textos Básicos, vol. 5. Secretaria de Minas e Energia da Bahia, Salvador-BA, pp. 43-103.

Xavier, R.P., 1991. The role of microstructural and fluid processes in the genesis of gold bearing shear zones: Fazenda Maria Preta mine, Rio Itapicuru greenstone belt, Bahia, Brazil. Ph.D. Thesis, University of Southampton, 241 p.

Xavier, R.P., Foster, R.P., 1999. Fluid evolution and chemical controls in the Fazenda Maria Preta (FMP) gold deposit, Rio Itapicuru Greenstone Belt, Bahia, Brazil. Chem. Geol. 154 (1-4), 133-154. 